\title{
VALUE DISTRIBUTION AND THE LEMMA OF THE LOGARITHMIC DERIVATIVE ON POLYDISCS
}

\author{
WILHELM STOLL \\ Department of Mathematics, University of Notre Dame \\ Notre Dame, Indiana 46556 U.S.A. \\ (Received June 18, 1983)
}

ABSTRACT. Value distribution is developed on polydiscs with the special emphasis that the value distribution function depend on a vector variable. A Lemma of the logarithmic derivative for meromorphic functions on polydiscs is derived. Here the Bergman boundary of the polydiscs is approached along cones of any dimension and exceptional sets for such an approach are defined.

KEY WORDS AND PHRASES. VaZue distribution theory, valence function, Jensen formula, characteristic function, counting function, spherical image, compensation function, Lemma of the Zogarithmic derivative, and approach cone.

1980 AMS MATHEMATICS SUBJECT CLASSIFICATION CODES. 32H30, $32 A 22$.

\section{INTRODUCTION.}

Value distribution for polydisc exhaustions has been studied by Ronkin [1], Stol1 [2] and others. They emphasized the growth of entire holomorphic and meromorphic functions and the representation of canonical functions to a given divisor in $\mathbb{C}^{\mathfrak{n}}$. For applications to mathematical logic, Lee A. Rubel and C. Ward Henson [3], [4] inquired if the Lemma of the logarithmic derivative could be established for a meromorphic function on a fixed given polydisc.

In the classical one variable theory, the Lemma of the logarithmic derivative has been one of the basic tools for a long time. In several variables, the analogous Lemma was proved only recently. For ball exhaustions of $\mathbb{C}^{\mathrm{n}}$, Vitter [ 5] proved the Lemma for differential operators with constant coefficients and derived the defect relation for meromorphic maps $f: \mathbb{C}^{\mathbf{n}} \rightarrow \mathbb{P}_{m}$. See also Stoll $[6]$. 
Vitter's Lemma extends easily to differential operators with polynomial or, with proper modifications, to differential operators with entire coefficients. Earlier, a weak version of the Lemma was proved by Gauthier and Hengartner [7] for the special operator $D^{\prime}=z_{1} \partial / \partial z_{1}+\ldots+z_{n} \partial / \partial z_{n}$, which does not permit the extension to a general differential operator. The differential operator $D^{\prime}$ suffices for meromorphic functions, but imposes unnecessary restrictions for meromorphic maps $\mathbf{f}: \mathbb{C}^{\mathbf{n}} \rightarrow \mathbb{P}_{\mathrm{m}}$. Recently, Shiffman has shown how to derive Vitter's Lemma from the result of Griffiths and King [8], which also can be interpreted as a Lemma of the logarithmic derivative. Biancofiore and Stoll [9] gave an elementary proof of Vitter's result. The same method will be used to obtain the Lemma of the logarithmic derivative for polydiscs.

A theory should reflect the intrinsic algebraic, geometric and analytic structures of the mathematical landscape under consideration. Our development of value distribution theory on polydiscs will adhere to this principle. This is an important feature of this paper, which is mostly self contained and requires only a minimal knowledge of several complex variables.

Let us outline the main result. The euclidean space $\mathbb{R}^{\mathbf{n}}$ is partially ordered by its coordinates. Denote by $\|\mathfrak{r}\|$ the length of $r$ in $\mathbb{R}^{n}$. For $z=\left(z_{1}, \ldots, z_{n}\right) \in \mathbb{C}^{n}$ define $|z|=\left(\left|z_{1}\right|, \ldots,\left|z_{n}\right|\right)$. For $0<b \in \mathbb{R}^{n}$ define

$$
\begin{aligned}
& \stackrel{+}{\mathbb{R}^{n}}(\boldsymbol{b})=\left\{\boldsymbol{r} \in \mathbb{R}^{\mathrm{n}} \mid 0<\boldsymbol{r}<\boldsymbol{b}\right\} \\
& \mathbb{D}(\mathfrak{b})=\left\{\boldsymbol{z} \in \mathbb{C}^{\mathbf{n}}|| \boldsymbol{z} \mid<\mathbf{b}\right\} \\
& \mathbb{D}\langle\mathbf{h}\rangle=\left\{\mathbf{z} \in \mathbb{C}^{\mathbf{n}}|| \mathbf{z} \mid=\mathbf{b}\right\} .
\end{aligned}
$$

Let $\Omega_{n}$ be the rotation invariant measure on the torus $\mathbb{D}\langle r\rangle$ for $r \stackrel{+}{n}^{n}(\mathfrak{b})$ such that $\mathbb{D}\langle\mathbf{r}\rangle$ has total volume 1 . In $\stackrel{+}{\mathbb{R}}^{n}(\mathbf{h})$, we could approach $\mathbf{b}$ via th with $t \rightarrow 1$ only. However Rubel is interested in the more general approach $r \rightarrow b$ with $0<\mathbf{r}<\mathbf{b}$. We introduce admissible approach cones $M$ with $1 \leq \operatorname{dim} M \leq n$ which contain both cases. On $M$ we introduce a measure $\Lambda_{M}$ such that $\Lambda_{M}(M)=\infty$. If $E \subseteq M$ with $\Lambda_{M}(E)<\infty$, then $b$ can be approached from $M-E$. 
Let $f$ be a non-constant meromorphic function on $\mathbf{D ( b )}$. The characteristic $T_{f}(r, q)$ of $f$ is a function of two points $r$ and $q$ in $\mathbb{R}^{n}(b)$. Take $\tau \in \mathbb{N}[1, n]$ and

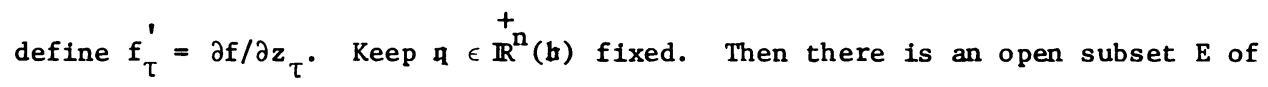
the manifold $M$ with $\Lambda_{M}(E)<\infty$ such that for all $r \in M-E$ with $r \geq q$ we have

$$
\int_{\mathbb{D}\langle\mathfrak{r}\rangle} \log ^{+}\left|\mathbf{f}_{\tau}^{\prime} / f\right| \Omega_{n} \leq 17 \log ^{+} T_{f}(\mathbf{r}, \mathbf{q})+19 \log +\frac{1}{\|\mathbf{b}-\mathbf{r}\|}
$$

\section{POLYDISCS.}

For any set $S$ let $S^{n}$ be the $n$-fold cartesian product. If $S \subseteq \mathbb{R}$ and if $-\infty \leq a<b \leq+\infty$, define

$$
\begin{array}{ll}
S[a, b]=\{x \in S \mid a \leq x \leq b\} & S(a, b)=\{x \in S \mid a<x<b\} \\
S(a, b]=\{x \in S \mid a<x \leq b\} & S[a, b)=\{x \in S \mid a \leq x<b\} \\
+ & S=S[0, \infty)=\{x \in S \mid x \geq 0\} .
\end{array}
$$

If $s \subseteq \mathbb{C}^{n}$, define $s_{*}=s-\{0\}$. If $x=\left(x_{1}, \ldots, x_{n}\right)$ and $y=\left(y_{1}, \ldots, y_{n}\right)$ are vectors in $\mathbb{R}^{n}$, define $x \leq y$ if $x_{j} \leq y_{j}$ for all $j \in \mathbb{N}[1, n]$ and $x<y$ if $x_{j}<y_{j}$ for all $j \in \mathbf{N}[1, n]$. If $s \subseteq \mathbb{R}^{n}$, define

$$
\begin{aligned}
& S[\mathbf{r}]=\{\boldsymbol{x} \in \mathrm{S} \mid \boldsymbol{x} \leq \boldsymbol{r}\} \quad \mathrm{S}(\boldsymbol{r})=\{\boldsymbol{x} \in \mathrm{S} \mid \boldsymbol{x}<\mathbf{r}\} . \\
& \text { For } z=\left(z_{1}, \ldots, z_{n}\right) \text { and } w=\left(w_{1}, \ldots, w_{n}\right) \text { in } \mathbb{c}^{n} \text {, and } p \in \mathbf{N} \text { and } \lambda \in \mathbb{C} \text { define } \\
& z+w=\left(z_{1}+w_{1}, \ldots, z_{n}+w_{n}\right) \quad z \cdot w=\left(z_{1} w_{1}, \ldots, z_{n} w_{n}\right) \\
& \lambda z=\left(\lambda z_{1}, \ldots, \lambda z_{n}\right) \quad z^{p}=\left(z_{1}^{p}, \ldots, z_{n}^{p}\right) \\
& |z|=\left(\left|z_{1}\right|, \ldots,\left|z_{n}\right|\right) \quad \bar{z}=\left(\bar{z}_{1}, \ldots, \bar{z}_{n}\right) \\
& \|z\|=\left(\left|z_{1}\right|^{2}+\ldots+\left|z_{n}\right|^{2}\right)^{1 / 2} \quad e^{z}=\left(e^{z_{1}}, \ldots, e^{z_{n}}\right)
\end{aligned}
$$




$$
\begin{aligned}
\log z & =\left(\log z_{1}, \ldots, \log z_{n}\right) & z^{\lambda}=e^{\lambda \log z} \quad \text { if } z \in\left(\mathbb{C}_{\star}\right)^{n} \\
0 & =0_{n}=(0, \ldots, 0) \in \mathbb{C}^{n} & 1_{n}=(1, \ldots, 1) \in \mathbb{C}^{n} .
\end{aligned}
$$

Here $\log$ is the principal value. For $r \in \mathbb{R}_{+}^{\mathrm{n}}$, define the polydiscs

$$
\mathbb{D}(\mathfrak{r})=\left\{z \in \mathbb{C}^{\mathbf{n}}|| z \mid<\mathfrak{r}\right\} \quad \mathbb{D}[\mathfrak{r}]=\left\{z \in \mathbb{C}^{\mathbf{n}}|| z \mid \leq \mathfrak{r}\right\} .
$$

The Bergman-Shilov boundary is given by

$$
\mathbb{D}\langle\mathfrak{r}\rangle=\left\{z \in \mathbb{C}^{\mathfrak{n}}|| z \mid=\mathfrak{r}\right\} .
$$

A surjective, real analytic map $\alpha_{\mathbf{r}}: \mathbb{R}^{n} \rightarrow \mathbb{D}\langle\mathbf{r}\rangle$ is defined by $\alpha_{\mathbf{r}}(\phi)=\mathfrak{r} \cdot \mathrm{e}^{\mathbf{i} \phi}$ for all $\phi \in \mathbb{R}^{\mathfrak{n}}$. If $\mathfrak{r}>0$, then $\mathbb{D}\langle\mathfrak{r}\rangle$ is an $\mathrm{n}$-dimensional, oriented, real analytic manifold such that $\alpha_{\mathfrak{r}}$ is an orientation preserving, local diffeomorphism which is bijective on $\mathbb{R}[0,2 \pi)^{\mathrm{n}}$.

For $\mathfrak{r}=1 \in \mathbb{R}$, we obtain the unit disc $\mathbb{D}=\mathbb{D}(1)$ with $\overline{\mathbb{D}}=\mathbb{D}[1]$ and $\partial \mathbb{D}=\mathbb{D}\langle 1\rangle$. For $\mathfrak{r} \in \stackrel{+}{\mathbb{R}^{n}}$, define the diagonal manifold

$$
\Delta(r)=\underset{t \in \mathbb{R}(0,1)}{U} \mathbb{D}\langle t r r\rangle \quad \Delta[r]=\underset{t \in \mathbb{R}[0,1]}{U} \mathbb{D}\langle t \mathbf{r}\rangle .
$$

A surjective, real analytic map $\beta_{\mathfrak{r}}: \mathbb{R}(0,1) \times \mathbb{R}^{\mathrm{n}} \rightarrow \Delta(\mathbf{r})$ is defined by $\beta_{\mathbf{r}}(t, \phi)=t \alpha_{\mathbf{r}}(\phi)$ for all $t \in \mathbb{R}(0,1)$ and $\phi \in \mathbb{R}^{\mathrm{n}}$. Moreover, $\Delta(\mathfrak{r})$ is an $(n+1)$-dimensional, oriented, real analytic manifold such that $\beta_{\mathfrak{r}}$ is an orientation preserving local diffeomorphism which is bijective on $\mathbb{R}(0,1) \times \mathbb{R}[0,2 \pi)^{\mathrm{n}}$. On $\left(\mathbb{C}_{*}\right)^{\mathrm{n}}$, we introduce the holomorphic differential forms

$$
\Omega_{\mathrm{n}}=\frac{1}{(2 \pi i)^{\mathrm{n}}} \frac{\mathrm{dz} 1}{\mathrm{z}_{1}} \wedge \ldots \wedge \frac{\mathrm{d} \mathrm{z}_{\mathrm{n}}}{\mathrm{z}_{\mathrm{n}}}
$$




$$
\xi_{\mathrm{n}}=\left(\frac{1}{2 \pi i}\right)^{\mathrm{n}-1} \sum_{\lambda=1}^{\mathrm{n}}(-1)^{\lambda-1}\left(\bigwedge_{\mu=1}^{\lambda-1} \frac{\mathrm{d} \mathrm{z}_{\mu}}{\mathrm{z}_{\mu}}\right) \wedge\left(\bigwedge_{\mu=\lambda+1}^{\mathrm{n}} \frac{\mathrm{d} \mathrm{z}_{\mu}}{\mathrm{z}_{\mu}}\right) .
$$

For $\mathbf{j} \in \mathbf{N}[1, \mathrm{n}]$ we have

$$
\frac{d z_{j}}{2 \pi i z_{j}} \wedge \xi_{n}=\Omega_{n}
$$

Let $j_{\mathfrak{r}}: \mathbb{D}\langle\mathfrak{r}\rangle \rightarrow\left(\mathbb{C}_{\star}\right)^{n}$ be the inclusion map. Then

$$
\begin{aligned}
\alpha_{r}^{*} j_{r}^{*}\left(\Omega_{n}\right) & =\frac{1}{(2 \pi)^{n}} d \phi_{1} \wedge \ldots \wedge d \phi_{n}>0 \\
\int_{\mathbb{D}\langle\mathfrak{r}\rangle} \Omega_{n} & =1 .
\end{aligned}
$$

Hence $j_{\mathfrak{r}}^{*}\left(\Omega_{\mathbf{n}}\right)$ is a positive measure on $\mathbb{D}\langle\mathbf{r}\rangle$ of total mass 1 such that $j_{\mathfrak{r}}^{*}\left(\Omega_{n}\right)$ is invariant under the action of $\mathbb{D}\left\langle 1_{n}\right\rangle$ on $\mathbb{D}\langle\mathfrak{r}\rangle$ defined by $\zeta(z)=\zeta \cdot z$ for $\zeta \in \mathbb{D}\left\langle 1_{n}\right\rangle$ and $\boldsymbol{z} \in \mathbb{D}\langle\mathbf{r}\rangle$. In particular, if $\mathbf{F}: \mathbb{D}\langle\mathbf{r}\rangle \rightarrow \mathbb{C}$ is integrable, then

$$
\int_{\mathbb{D}\langle\mathfrak{r}\rangle} F \Omega_{n}=\int_{z \in \mathbb{D}\langle\mathfrak{r}\rangle}\left(\frac{1}{2 \pi} \int_{0}^{2 \pi} F\left(e^{i \phi_{z}}\right) d \phi\right) \Omega_{n}(z) .
$$

Let $X$ be a form of bidegree $(1,1)$ on an open subset $U \neq \emptyset$ of $\mathbb{C}^{\mathrm{n}}$ where

$$
x=\frac{i}{2 \pi} \sum_{\mu, \nu=1}^{\mathrm{n}} x_{\mu \bar{\nu}} \mathrm{dz}{ }_{\mu} \wedge \overline{\mathrm{d}}_{\nu}
$$

Then $X$ is said to be non-negative (respectively positive) at $z \in U$ if for all $0 \neq x \in \mathbb{C}^{\mathrm{n}}$ we have

$$
\sum_{\mu, \nu=1}^{\mathbf{n}} \chi_{\mu \bar{\nu}}(z) \mathrm{x}_{\mu} \overline{\mathbf{x}}_{\nu} \geq 0 \quad(\text { respectively }>0) .
$$


If $S \subseteq U$ and $X$ is non-negative (respectively positive) at every point of $S$ we say that $X$ is non-negative on $S$ (respectively positive on $S$ ) in signs $X \geq 0$ on $S$ (respectively $X>0$ on $S$ ). On $U \cap\left(\mathbb{c}_{*}\right)^{n}$ we have

$$
x \wedge \xi_{n}=\sum_{\mu, \nu=1}^{n} x_{\mu \nu} z_{\mu} \bar{d}_{\nu} \wedge \Omega_{n}
$$

Take $r \in \mathbb{R}^{\mathbf{n}}$ and assume that $U \supset \Delta(\boldsymbol{r})$. Let $\mathrm{l}_{\mathbf{r}}: \Delta(\boldsymbol{r}) \rightarrow U$ be the inclusion map. For $t \in \mathbb{R}(0,1)$ and $\phi=\left(\phi_{1}, \ldots, \phi_{\mathbf{n}}\right) \in \mathbb{R}^{\mathbf{n}}$ we have

$$
\beta_{r}^{*}{ }^{*}\left(x \wedge \xi_{n}\right)(t, \phi)=\left(\frac{1}{2 \pi}\right)^{n} \sum_{\mu, \nu=1}^{n} x_{\mu \nu}\left(t r \cdot e^{i \phi}\right) r_{\mu} r_{\nu} e^{i\left(\phi_{\mu}-\phi_{\nu}\right)} 2 t d t \wedge d \phi
$$

where $\mathrm{d} \phi=\mathrm{d} \phi_{1} \wedge \ldots \wedge \mathrm{d} \phi_{\mathrm{n}}$. In particular

$$
\begin{aligned}
& { }_{r}^{*}\left(X \wedge \xi_{n}\right) \geq 0 \text { if } \chi \geq 0 \text { on } \Delta(r) \\
& { }_{r}^{*}\left(X \wedge \xi_{n}\right)>0 \text { if } X>0 \text { on } \Delta(r) .
\end{aligned}
$$

For $0 \neq z \in \mathbb{c}^{n}$, define $\ell_{z}: \mathfrak{c} \rightarrow \mathbb{C}^{n}$ by $\ell_{z}(u)=u z$. If $z \in \mathbb{D}\langle\mathbf{r}\rangle$, then $\ell_{z}(\mathbb{C}(1)-\{0\}) \subseteq \Delta(r)$. The identities (2.6) and (2.10) and Fubini's theorem imply easily :

LEMMA 2.1. Take $r \in \stackrel{+}{n}^{n}$. Let $\chi$ be a form of bidegree $(1,1)$ on an open neighborhood $U$ of $\Delta(r)$. Assume that ${ }^{*}{ }_{r}^{*}\left(\chi \wedge \xi_{n}\right)$ is integrable over $\Delta(r)$. Then

$$
\int_{\Delta(\mathfrak{r})} x \wedge \xi_{n}=\int_{z \in \mathbb{D}\langle\mathfrak{r}\rangle}\left(\int_{\mathbb{D}} l_{z}^{*}(x)\right) \Omega_{\mathbf{z}}(z)
$$

COROLLARY 2.2. Take $\mathbf{r} \in \stackrel{+}{\mathbb{R}^{\mathrm{n}}}$ and $\mathbf{r} \in \stackrel{+}{\mathbb{R}}$. Let $X$ be a form of bidegree $(1,1)$ on an open neighborhood $U$ of $\Delta(r \mathfrak{r})$. Assume that $\imath_{r r}^{*}\left(\chi \wedge \xi_{n}\right)$ is integrable over $\Delta(r r)$. Then 


$$
\int_{\Delta(\mathbf{r} r)} x^{\wedge} \xi_{\mathbf{n}}=\int_{x \in \mathbb{D}\langle\mathfrak{r}\rangle}\left(\int_{\mathbb{D}(r)} e_{x}^{*}(x)\right) \Omega_{n}(x) .
$$

Take $n \in \mathbb{N}$ and $p \in \mathbb{Z}[0, n]$. Let $\mathbb{C}(p, n)$ be the set of all injective increasing maps $\tau: \mathbb{N}[1, p] \rightarrow \mathbf{N}[1, n]$. If $p=0$, then $\mathbb{e}(p, n)=\emptyset$. If $p \in \mathbb{N}[1, n]$, let $l_{p}: N[1, p]+N[1, n]$ be the inclusion map. If $p=n$, then $\mathbb{E}(n, n)=\left\{l_{n}\right\}$. For $\tau \in \mathbb{E}(p, n)$, we assign various operators.

The complement $\tilde{\tau} \in \mathbb{C}(n-p, n)$ is uniquely defined by $\operatorname{Im} \tau n \operatorname{Im} \tilde{\tau}=\emptyset$ or by Im $\tau \cup \operatorname{Im} \tilde{\tau}=\mathbf{N}[1, \mathrm{n}] . \quad$ Clearly $\tilde{\tau}=\tau$ and $\tilde{\imath}_{\mathrm{n}}=\emptyset$.

A surjective linear map $\pi_{\tau}: \mathbb{c}^{\mathrm{n}} \rightarrow \boldsymbol{d}^{\mathrm{p}}$ called the $\tau$-projection is defined by

$$
\pi_{\tau}(z)=\left(z_{\tau(1)}, \cdots, z_{\tau(p)}\right) \in \mathbb{c}^{p}
$$

Let $\delta_{j k}$ be the Kronecker symbol. Define $p_{j}=\left(\delta_{j 1}, \ldots, \delta_{j n}\right)$. For each $z \in \mathbb{c}^{n-p}$ an injective, affine map $\tau_{z}: \mathbb{e}^{p} \rightarrow \mathbb{C}^{n}$ called the $\tau$-injection at $z$ is defined by

$$
\tau_{z}(\mathfrak{m})=\sum_{\mu=1}^{p} w_{\mu}{ }^{\mathfrak{p}} \tau(\mu)+\sum_{\nu=1}^{n-p} z_{\nu}^{\mathfrak{p}} \tilde{\tau}(\nu) .
$$

If $z=0 \in \mathbb{c}^{\mathrm{n}-\mathrm{p}}$, then $\tau_{0}$ is linear. If $\lambda>0$ and $\boldsymbol{u}$ and $\boldsymbol{w}$ belong to $\mathbb{c}^{\mathrm{p}}$, then

$$
\tau_{0}(\mathfrak{u} \cdot \mathfrak{w})=\tau_{0}(\mathfrak{u}) \cdot \tau_{0}(\mathfrak{w}) \quad \tau_{0}\left(\mathfrak{u}^{\lambda}\right)=\tau_{0}(\mathfrak{u})^{\lambda} .
$$

If $z \in \mathbb{C}^{n}$, then one and only one number $p \in \mathbb{Z}[0, n]$ and map $\sigma_{z} \in \mathbb{C}(p, n)$ exist such that $z_{\sigma_{z}(j)} \neq 0$ for $j=1, \ldots, p$ and $z_{\tilde{\sigma}_{z}(j)}=0$ for $j=1, \ldots, n-p$. The map $\sigma_{z}$ is called the support of $z$. Obviously $p=0$ iff $z=0$. Also

$$
\operatorname{Im} \sigma_{x \cdot y}=\operatorname{Im} \sigma_{x} \cap \operatorname{Im} \sigma_{y}
$$

Take $r \in \mathbb{R}^{\mathrm{n}}$. Let $\mathrm{F}$ be a function on $\mathbf{D}\langle\boldsymbol{r}\rangle$. Define 


$$
\mathfrak{A}(r, F)=\frac{1}{(2 \pi)^{n}} \int_{0}^{2 \pi} \cdots \int_{0}^{2 \pi} F\left(r_{1} e^{i \phi_{1}}, \ldots, r_{n} e^{i \phi_{n}}\right) d \phi_{1} \ldots d \phi_{n}
$$

provided the integral exists (perhaps in the sense of summability where $+\infty$ or $-\infty$ are permitted). If $\mathfrak{r}>0$, then

$$
\mathbb{H}(\mathbf{r}, F)=\int_{\mathbb{D}\langle\mathfrak{r}\rangle} F \Omega_{\mathbf{n}} .
$$

If $0 \leq \mathfrak{r} \neq 0$, then $\sigma_{\mathfrak{r}} \in \mathbb{C}(p, n)$ with $\mathrm{p} \in \mathbb{N}[1, n]$. Take $0 \in \mathbb{C}^{\mathrm{n}-\mathrm{p}}$. Abbreviate $\sigma=\sigma_{r} \cdot$ Then

$$
\mathfrak{H l}(\mathfrak{r}, F)=\int_{\mathbb{D}\left\langle\pi_{\sigma}(\mathfrak{r})\right\rangle}\left(F \circ \sigma_{0}\right) \Omega_{\mathrm{p}} .
$$

If $\mathfrak{r}=0$, then

$$
\mathfrak{a}(0, F)=F(0) \text {. }
$$

Take $p \in \mathbb{N}[1, n]$ and $\tau \in \mathbb{C}(p, n)$. Take $z \in \mathbb{D}\langle\pi \tilde{\tau}(\mathfrak{r})\rangle$, then $\tau_{\boldsymbol{z}}\left(\mathbb{D}\left\langle\pi \pi_{\tau}(\mathfrak{r})\right\rangle\right) \subseteq \mathbb{D}\langle\mathfrak{r}\rangle$. Hence $F \circ \tau_{z}$ is defined on $\mathbb{D}\left\langle\pi_{\tau}(r)\right\rangle$. By Fubini's theorem $\mathfrak{A l}\left(\pi_{\tau}(\mathfrak{r}), F \circ \tau_{z}\right)=H(z)$ exists for almost all $\boldsymbol{z} \in \mathbb{D}\langle\pi \sim \tilde{\tau}(\boldsymbol{r})\rangle$ and we have

$$
\mathfrak{M}(\mathfrak{r}, F)=\mathfrak{M}(\pi \tilde{\tau}(\mathfrak{r}), H)
$$

More explicitly, this is written as

$$
\mathfrak{H}(\mathfrak{r}, F)=\mathfrak{H}\left(\pi \tilde{\tau}(\mathfrak{r}), \mathfrak{H}\left(\pi_{\tau}(\mathfrak{r}), F \circ \tau_{z}\right)\right)
$$

If $\mathfrak{r}>0$, then

$$
\int_{\mathbb{D}\langle\mathfrak{r}\rangle} F \Omega_{n}=\int_{z \in \mathbb{D}\left\langle\pi \tau_{\tau}(\mathfrak{r})\right\rangle}\left(\int_{\mathbb{D}\left\langle\pi_{\tau}(\mathfrak{r})\right\rangle} F \circ \tau_{z} \Omega_{p}\right) \Omega_{n-p}(z) .
$$

Sometimes we shall write $\mathfrak{A}(\mathfrak{r}, F)$ as in (2.18) even if $\mathfrak{r}$ has some zero coordinates. Then (2.21) writes as in (2.22) which is more instructive. 


\section{PLURISUBHARMONIC FUNCTIONS.}

Let $B$ be a subset of $\mathbb{R}_{+}^{n}$. A function $g: B \rightarrow \mathbb{R} \cup\{-\infty\}$ is said to be $\underline{\text { increasing }}$ if $g(x) \leq g(y)$ whenever $\boldsymbol{x} \in \mathrm{B}$ and $\mathfrak{y} \in \mathrm{B}$ with $\boldsymbol{x} \leq \boldsymbol{y}$. The set $\mathrm{B}$ is said to be $\underline{\text { logarithmic convex }}$ if $\boldsymbol{x} \in \mathrm{B}$ and $\boldsymbol{y} \in \mathrm{B}$ and $\lambda \in \mathbb{R}(0,1)$ implies $\boldsymbol{x}^{\lambda} \mathbf{y}^{1-\lambda} \in \mathrm{B}$. The function $g$ is said to be logarithmic convex, if $B$ is logarithmic convex and if $\boldsymbol{X} \in \mathrm{B}$ and $y \in B$ and $\lambda \in \mathbb{R}(0,1)$ imply

$$
g\left(x^{\lambda}{ }^{1-\lambda}\right) \leq \lambda g(x)+(1-\lambda) g(y) .
$$

Here we are mostly interested in the case where $B$ is a polydisc. Take $b \in \mathbb{R}^{+}$.

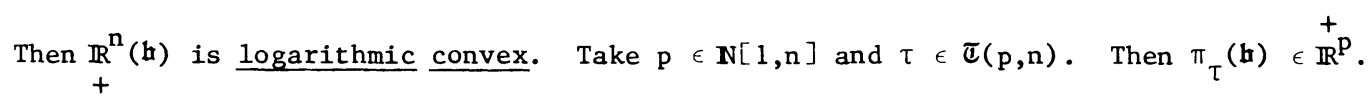
If $0 \in \mathbb{C}^{\mathrm{n}-\mathrm{p}}$, an injective linear map $\tau_{0}: \mathbb{C}^{\mathrm{p}} \rightarrow \mathbb{C}^{\mathrm{n}}$ is defined which maps $\mathbb{R}_{+}^{\mathrm{p}}\left(\pi_{\tau}(\mathfrak{b})\right)$ into $\underset{+}{\mathbb{R}^{\mathrm{n}}(\mathfrak{h})}$. Define $\beta_{\tau}=\log \pi_{\tau}(\mathfrak{b}) \in \mathbb{R}^{\mathrm{p}}$. Given a function $\mathrm{g}: \mathbb{R}_{+}^{\mathrm{n}}(\mathbf{b}) \rightarrow \mathbb{R} \cup\{-\infty\}$ define

$$
h_{\tau}: \mathbb{R}^{p}\left(\beta_{\tau}\right) \rightarrow \mathbb{R} \cup\{-\infty\} \quad \text { by } \quad h_{\tau}(x)=g\left(\tau_{0}\left(e^{x}\right)\right)
$$

LEMMA 3.1. If $g$ is logarithmic convex, then $h_{\tau}$ is convex.

PROOF. Take $\boldsymbol{x}$ and $\mathfrak{y}$ in $\mathbb{R}^{\mathrm{P}}\left(\beta_{\tau}\right)$. Take $\lambda \in \mathbb{R}(0,1)$. Define $\mu=1-\lambda$. Then

$$
\begin{aligned}
h_{\tau}(\lambda \boldsymbol{x}+\mu \boldsymbol{y}) & =g\left(\tau_{0}\left(e^{\lambda \boldsymbol{x}} e^{\mu \boldsymbol{y}}\right)\right)=g\left(\tau_{0}\left(e^{\mathbf{x}}\right){ }^{\lambda} \tau_{0}\left(e^{\mathbf{y}}\right)^{\mu}\right) \\
& \leq \lambda g\left(\tau_{0}\left(e^{\boldsymbol{x}}\right)\right)+\mu g\left(\tau_{0}\left(e^{\mathfrak{y}}\right)\right)=\lambda_{\tau}(\boldsymbol{x})+\mu h_{\tau}(\mathbf{y}) \quad \text { q.e.d. }
\end{aligned}
$$

Observe, if $\tau=l_{n}$, then $\tau_{0}\left(\mathbb{R}^{n}\left(\beta_{\tau}\right)\right)=\stackrel{+}{\mathbb{R}^{n}}(b)$ is the interior of $\mathbb{R}_{+}^{n}(b)$. In particular $g\left(e^{x}\right)$ is convex and $g$ is continuous on $\mathbb{R}^{n}(b)$, if $g$ is logarithmic convex. LEMMA 3.2. If $h_{\tau}$ is convex for each $\tau \in \mathbb{C}(p, n)$ and $p \in \mathbb{N}[1, n]$ then (3.1) holds for all $\begin{gathered}\boldsymbol{x} \in \mathbb{R}^{\mathrm{n}}(\mathfrak{h}) \\ +\end{gathered}$ and $\mathfrak{y} \in \underset{+}{\mathbb{R}^{\mathrm{n}}(\mathfrak{b})}$ with $\sigma_{\boldsymbol{x}}=\sigma_{\mathfrak{y}}$ and for all $\lambda \in \mathbb{R}(0,1)$. 
PROOF. Define $z=x^{\lambda}{ }^{1-\lambda}$. Then $\sigma_{z}=\sigma_{x}=\sigma_{y}=\tau$ where the $j^{\text {th }}$ coordinate of the vectors $\boldsymbol{z}, \boldsymbol{x}$ or $\boldsymbol{y}$ is positive if and only if $\boldsymbol{j} \in \operatorname{Im} \tau$. Hence $\tilde{\boldsymbol{x}}$ and $\tilde{\boldsymbol{y}}$ exist in $\mathbb{R}^{\mathrm{p}}\left(\beta_{\tau}\right)$ such that $\boldsymbol{x}=\tau_{0}\left(\mathrm{e}^{\tilde{\boldsymbol{x}}}\right)$ and $\boldsymbol{y}=\tau_{0}\left(\mathrm{e}^{\tilde{\boldsymbol{y}}}\right)$. Define $\mu=1-\lambda$. Then

$$
\begin{aligned}
& g\left(\boldsymbol{x}^{\lambda} \hat{y}^{\mu}\right)=g\left(\tau_{0}\left(e^{\tilde{\boldsymbol{x}}}\right)^{\lambda} \tau_{0}\left(e^{\tilde{\mathbf{y}}}\right)^{\mu}\right)=g\left(\tau_{0}\left(e^{\lambda \tilde{\boldsymbol{x}}+\mu \tilde{\mathbf{y}}}\right)\right)=h_{\tau}(\lambda \tilde{\boldsymbol{x}}+\mu \tilde{\mathbf{y}}) \\
& \leq \lambda h_{\tau}(\tilde{x})+\mu h_{\tau}(\tilde{y})=\lambda g\left(\tau_{0}\left(e^{\tilde{x}}\right)\right)+\mu g\left(\tau_{0}\left(e^{\tilde{y}}\right)\right)=\lambda g(x)+\mu g(y) \quad q \cdot e \cdot d .
\end{aligned}
$$

If $\sigma_{\boldsymbol{x}} \neq \sigma_{\mathfrak{y}}$, the conclusion of Lemma 3.2 may be incorrect: Take $\mathrm{n}=2$ and $\mathbf{h}=(2,2)$. Define $\mathrm{g}(\mathrm{x}, \mathrm{y})=1$ if $\mathrm{x}=0$ or $\mathrm{y}=0$ and $\mathrm{g}(\mathrm{x}, \mathrm{y})=0$ if $0<\mathrm{x}<2$ and $0<y<2$. Each function $h_{\tau}$ is constant and therefore convex. If $0<\lambda<1$, then $(0,0)^{\lambda}(1,1)^{1-\lambda}=(0,0)$ and

$$
g\left((0,0)^{\lambda}(1,1)^{1-\lambda}\right)=g(0,0)=1>\lambda=\lambda g(0,0)+(1-\lambda) g(1,1) .
$$

However we have the following result:

LEMMA 3.3. If $g$ is increasing on $\mathbb{R}_{+}^{n}(\mathfrak{h})$ and if $h_{\tau}$ is convex on $\mathbb{R}^{p}\left(\beta_{\tau}\right)$ for all $\tau \in \mathbb{C}(p, n)$ and $p \in \mathbf{N}[1, n]$, then $g$ is logarithmic convex.

PROOF. If $x^{\lambda} y^{1-\lambda}=0$, then (3.1) is trivial since $g(0) \leq g(x)$ and $g(0) \leq g(y)$. Define $\mu=1-\lambda$ and $z=x^{\lambda}{ }^{\mu}$. Then $\operatorname{Im} \sigma_{z}=\operatorname{Im} \sigma_{x} \wedge \operatorname{Im} \sigma_{y}$. Put $\tilde{x}_{j}=0=\tilde{y}_{j}$ if $\mathrm{j} \notin \operatorname{Im} \sigma_{z}$ and $\tilde{x}_{j}=x_{j}>0$ and $\tilde{y}_{j}=y_{j}>0$ if $j \in \operatorname{Im} \sigma_{z}$. Then $\tilde{x}=\left(\tilde{x}_{1}, \ldots, \tilde{x}_{n}\right) \in \mathbb{R}_{+}^{n}(b)$ and $\tilde{\mathfrak{y}}=\left(\tilde{y}_{1}, \ldots, \tilde{y}_{n}\right) \in \underset{+}{\mathbb{R}^{n}(\mathfrak{b})}$ with $\sigma_{\tilde{x}}=\sigma_{\mathfrak{y}}=\sigma_{\mathfrak{z}}$ and $\tilde{x} \leq \boldsymbol{x}$ and $\tilde{\mathfrak{y}} \leq \mathbf{y}$. Also we have $z=\tilde{\boldsymbol{x}}^{\lambda} \tilde{\mathbf{y}}^{\mu}$. Lemma 3.2 implies

$$
g\left(x^{\lambda}{ }^{\mu}\right)=g\left(\tilde{x}^{\lambda} \tilde{y}^{\mu}\right) \leq \lambda g(\tilde{x})+\mu g(\tilde{y}) \leq \lambda g(x)+\mu g(\mathfrak{y})
$$
$q \cdot e \cdot d$.

PROPOSITION 3.4. Take $b \in \stackrel{+}{\mathbb{R}^{n}}$. An upper semi-continuous function $g: \mathbb{R}^{\mathrm{n}}(\boldsymbol{b}) \rightarrow \mathbb{R} \cup\{-\infty\}$ increases and is logarithmic convex if and only if the function $\mathrm{u}: \mathbb{D}(\boldsymbol{b}) \rightarrow \mathbb{R} \cup\{-\infty\}$ defined by $\mathrm{u}(\boldsymbol{z})=\mathrm{g}(|\mathbf{z}|)$ is plurisubharmonic. 
PROOF. If $u$ is plurisubharmonic, a theorem of Vladimirov [10] p. 88 (see also Ronkin [1] Theorem 2.1.2) implies that $g$ increases and that all $h_{\tau}$ are convex. Hence $\mathrm{g}$ increases and is logarithmic convex. If $\mathrm{g}$ increases and is logarithmic convex, then $g$ increases and all $h_{\tau}$ are convex. By vladimirov's theorem $u$ is plurisubharmonic, q.e.d.

Take $\mathfrak{b} \in \stackrel{+}{\mathbb{R}^{n}}$. Let $\mathrm{u}$ be a plurisubharmonic function on $\mathbb{D}(\mathfrak{b})$. For $\mathfrak{r} \in \underset{+}{\mathbb{R}^{n}}(\mathfrak{b})$ define

$$
\begin{aligned}
& M(\mathfrak{r}, u)=\operatorname{Max}\{u(z) \mid z \in \mathbb{D}\langle\mathfrak{r}\rangle\} \\
& \mathscr{H l}(\mathfrak{r}, u)=\frac{1}{(2 \pi)^{\mathrm{n}}} \int_{0}^{2 \pi} \cdots \int_{0}^{2 \pi} u\left(r \mathrm{e}^{i \phi}\right) \mathrm{d} \phi_{1} \ldots \mathrm{d} \phi_{\mathrm{n}} .
\end{aligned}
$$

Then $M(|z| ; u)$ and $\mathfrak{A l}(|z|, u)$ are plurisubharmonic functions of $z$ on $\mathbb{D}(\mathbf{b})$ (Ronkin [1] pages 75 and 84$)$. Therefore $M(r, u)$ and $\mathfrak{A}(\mathbf{r}, u)$ increase and are logarithmic convex functions of $r$ in $\mathbb{R}^{n}(\mathfrak{b})$. If $u \neq-\infty$, then $M(\mathfrak{r}, u)$ and $\mathbb{A l}(\mathfrak{r}, u)$ are real numbers for all $\mathfrak{r} \in \mathbb{R}^{n}(\mathfrak{r})$. If some coordinates of $\mathfrak{r}$ are zero, the same remains true if $u \circ \sigma_{\mathfrak{r}_{0}} \neq-\infty$. PROPOSITION 3.5. Take $\mathfrak{b} \in \stackrel{+}{\mathbb{R}^{n}}$. Let $u: \mathbb{D}(\mathfrak{b}) \rightarrow \mathbb{R}$ be a pluriharmonic function. Then $\mathfrak{A}(\mathfrak{r}, u)=u(0)$ for all $\mathfrak{r} \in \mathbb{R}_{+}^{\mathrm{n}}(\mathfrak{b})$.

PROOF. Since $u$ and $-u$ are plurisubharmonic we have

$$
u(0) \leq \mathfrak{H}(\mathbf{r}, u)=-\mathbb{H}(\mathbf{r},-u) \leq-(-u(0))=u(0) \quad \text { q.e.d. }
$$

A function $g: \mathbb{D}(\mathfrak{h}) \rightarrow \mathbb{R} \cup\{-\infty,+\infty\}$ is said to be quasipluriharmonic if there are plurisubharmonic functions $u \neq-\infty$ and $v$ 丰 $-\infty$ such that $g=u-v$. Strictly speaking $g$ is defined except for a pluripolar set

$$
I_{g} \subseteq\{z \mid u(z)=-\infty=v(z)\}
$$

called the indeterminacy. For $0<\mathfrak{r}<\mathbf{b}$, the integral average

$$
\mathfrak{H}(\mathbf{r}, \mathrm{g})=\mathfrak{H}(\mathbf{r}, \mathrm{u})-\mathfrak{H}(\mathbf{r}, \mathrm{v})
$$

is well defined. 
Let $\boldsymbol{g}(\mathbf{b})$ be the real vector space of all quasipluriharmonic functions on $\mathbb{D}(\mathbf{b})$. Then the set $\mathfrak{p}(\mathfrak{b})$ of all pluriharmonic functions on $\mathbb{D}(\mathfrak{b})$ is a linear subspace of $\boldsymbol{g}(\mathfrak{b})$. Let $\mathfrak{Q}(\mathfrak{b})=\boldsymbol{g}(\mathfrak{b}) / \mathfrak{p}(\mathfrak{b})$ be the quotient vector space and let $\rho: g(\mathfrak{b}) \rightarrow \mathfrak{Q}(\mathfrak{b})$ be the residual map. An element of $\mathcal{Q}(\mathfrak{b})$ is called a quasipluriharmonic class on $\mathbb{D}(\mathfrak{b})$. Take $h \in \mathbb{Q}(\mathfrak{h})$. Then $h=\rho(g)$ with $g \in \mathbb{g}(\mathfrak{h})$. If $h=\rho(\tilde{g})$ with $\tilde{g} \in \mathbb{g}(\mathbf{b})$ then $\tilde{g}=g+v$ where $v \in \mathfrak{p}(\mathfrak{h})$. Take $\mathbf{r} \in \stackrel{+}{\mathbb{R}}^{n}(\mathfrak{h})$ and $\mathfrak{q} \in \stackrel{+}{\mathbb{R}}^{n}(\mathfrak{b})$. Then

$$
\begin{aligned}
& \mathfrak{H}(\mathfrak{r}, \tilde{g})=\mathfrak{M}(\mathfrak{r}, g)+v(0) \\
& \mathfrak{H}(\mathfrak{q}, \tilde{g})=\mathfrak{M}(\mathfrak{q}, g)+v(0)
\end{aligned}
$$

Subtraction implies

$$
\mathfrak{H}(\mathfrak{r}, g)-\mathfrak{M}(\mathfrak{q}, g)=\mathfrak{H}(\mathfrak{r}, \tilde{g})-\mathfrak{H}(\mathfrak{q}, \tilde{g})
$$

Therefore the valence function of $\mathrm{h}$ is well defined independent of the choice of the representative $g$ by

$$
\mathbb{N}(h, \mathfrak{r}, \mathfrak{q})=\mathfrak{M}(\mathfrak{r}, g)-\mathfrak{M}(\mathfrak{q}, g) \in \mathbb{R}
$$

for $0<\mathfrak{r}<\mathfrak{h}$ and $0<\mathfrak{q}<\mathfrak{h}$. For fixed $\mathfrak{r}$ and $\mathfrak{q}$, the function $\mathfrak{N}(\mathfrak{h}, \mathfrak{r}, \mathfrak{h})$ is 1 inear in $h$. If $r, \mathfrak{q}, \mathfrak{p}$ belong to $\stackrel{+}{\mathbb{R}}^{n}(\mathfrak{r})$, then

$$
\begin{aligned}
\mathbb{N}(h, \mathfrak{r}, \mathfrak{q})+\mathbb{N}(h, \mathfrak{q}, \mathfrak{p}) & =\mathbb{N}(h, \mathfrak{r}, \mathfrak{p}) \\
\mathbb{N}(\boldsymbol{h}, \mathfrak{r}, \mathfrak{q}) & =-\mathbb{N}(\mathbf{h}, \mathfrak{h}, \mathfrak{r}) .
\end{aligned}
$$

The set $\mathscr{S}_{+}(\mathfrak{b})$ of all plurisubharmonic functions $u \neq-\infty$ on $\mathbb{D}(\mathfrak{b})$ is contained in $\mathscr{S}(b)$ and $\mathscr{S}_{+}(b)$ is closed under addition and multiplication with non-negative numbers. Define $\mathbb{Q}_{+}(\mathfrak{h})=\rho\left(\mathscr{S}_{+}(\mathfrak{b})\right)$ in $\mathbb{Q}(\mathfrak{h})$. Each $g \in \mathbb{Q}_{+}(\mathfrak{h})$ is called a plurisubharmonic class. If $u \in \underline{S}_{+}(\mathbf{b})$ such that $\rho(u)=\mathbf{g}$ and if $0<\mathfrak{r}<\mathbf{b}$ and $0<\mathfrak{q}<\mathbf{b}$, then

$$
\mathbb{N}(g, r, \mathfrak{q})=\mathfrak{H}(\mathbf{r}, u)-\mathfrak{H}(\mathfrak{q}, u)
$$

The definition extends to all $\mathfrak{q} \in \underset{+}{\mathbb{R}^{n}(\mathfrak{h})}$ with $u \circ \sigma_{\mathfrak{q}, 0} \neq-\infty$, and the extension does not depend on the choice of $u$. The function $\mathbb{N}(g, \mathfrak{r}, \mathfrak{q})$ increases in $r$ and decreases in $\mathfrak{q}$. 
If $0 \leq \mathfrak{q} \leq \mathfrak{r}<\mathfrak{h}$, then

$$
\mathbb{N}(\boldsymbol{g}, \mathfrak{r}, \mathfrak{q}) \geq 0
$$

If $\mathfrak{q}$ is fixed and $\boldsymbol{r}$ converges to $\mathfrak{b}$, then $\mathbb{N}(g, \mathfrak{r}, \mathfrak{q})$ measures the growth of $g$.

Now, we will give examples of valence functions of divisors, meromorphic maps and meromorphic functions.

Take $\mathbf{h} \in \stackrel{+}{\mathbb{R}}^{11}$. Let $\mathfrak{O}(\mathbf{b})$ be the integral domain of all holomorphic functions on $\mathbb{D}(\mathfrak{b})$. Let $\mathbb{K}(\mathfrak{b})$ be the field of meromorphic functions on $\mathbb{D}(\mathfrak{b})$. Then $\mathbb{K}(\mathfrak{b})$ is the field of quotients of $\mathfrak{O}(\mathfrak{h})$. Define $\mathbb{K}_{\star}(\mathfrak{h})=\mathbb{K}(\mathfrak{h})-\{0\}$ and let

$$
\mathbb{O}^{*}(\mathfrak{h})=\{\mathbf{f} \in \mathbb{O}(\mathfrak{h}) \mid \mathbf{f}(\boldsymbol{z}) \neq 0 \text { for all } \boldsymbol{z} \in \mathbb{D}(\mathfrak{b})\}
$$

be the multiplicative group of units in $\mathfrak{O}(\mathfrak{b})$. The quotient group $\mathbb{D}(\mathfrak{b})=\mathfrak{K}_{\star}(\mathfrak{b}) / \mathbb{O}^{*}(\mathfrak{b})$ is written additively and is called the module of divisor on $\mathbb{D}(\mathfrak{h})$. Let $\mu: \mathfrak{K}_{\star}(\mathfrak{b}) \rightarrow \mathfrak{D}(\mathfrak{b})$ be the residual map. Define $\mathfrak{O}_{\star}(\mathfrak{b})=\mathfrak{O}(\mathfrak{b})-\{0\}$. Then $\mathbb{D}^{+}(\mathbf{b})=\mu\left(\mathfrak{O}_{\star}(\mathfrak{b})\right)$ is the additively closed subset of non-negative divisors. We write the variable as an index, $\mu_{f}=\mu(f)$ for all $f \in \mathbb{K}_{\star}(\mathfrak{b})$. If $\mu_{f}$ is non-negative, we write $\mu_{f} \geq 0$. If $f \in \mathbb{K}_{\star}(\mathrm{h})$, then $\mathrm{f}=\mathrm{g} / \mathrm{h}$ where $\mathrm{g} \neq \mathrm{f} 0$ and $\mathrm{h} \neq \mathrm{f} 0$ are holomorphic functions on $\mathbb{D}(\mathbf{h})$. Therefore $\log |\mathrm{f}|=\log |\mathrm{g}|-\log |\mathrm{h}| \in \mathbb{S}(\mathrm{b})$ and the map $\mathrm{f} \mapsto \log |\mathrm{f}|$

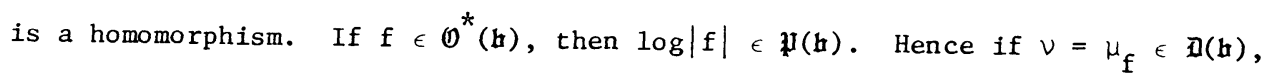
the valence function of the divisor $v$

$$
N_{V}(\mathbf{r}, \mathbf{q})=\mathbb{N}(\rho(\log |\mathbf{f}|), \mathbf{r}, \mathbf{q})
$$

is well defined independent of the representative $f$ with $\nu=\mu_{f}$ obviously, if $0<\mathfrak{r}<\mathfrak{h}$ and $0<\mathfrak{q}<\mathfrak{b}$, the definition (3.12) is also known as the Jensen formula

$$
N_{\mu_{f}}(\mathfrak{r}, \mathfrak{q})=\int_{\mathbb{D}\langle\mathfrak{r}\rangle} \log |f| \Omega_{n}-\int_{\mathbb{D}\langle\mathfrak{q}\rangle} \log |f| \Omega_{n} .
$$

The map $\nu \rightarrow N_{V}(\mathfrak{r}, \mathfrak{q})$ is an additive homomorphism. If $\mathfrak{r}, \mathfrak{p}, \mathfrak{q}$ belong to $\mathbb{R}^{n}(\mathfrak{b})$, then

$$
N_{V}(\mathfrak{r}, \mathfrak{q})+N_{V}(\mathfrak{q}, \mathfrak{p})=N_{V}(\mathfrak{r}, \mathfrak{p})=-N_{V}(\mathfrak{p}, \mathfrak{r}) \text {. }
$$


If $\nu \in \mathbb{D}^{+}(\mathfrak{b})$, then $N_{v}(\mathfrak{r}, \mathfrak{h})$ increases and is logarithmic convex in $\mathfrak{r}$ with

$$
N_{\nu}(\mathfrak{r}, \mathfrak{q}) \geq 0 \quad \text { if } 0 \leq \mathfrak{q} \leq \mathfrak{r}<\mathfrak{h} \text {. }
$$

Naturally, if we consider divisors on complex manifolds and spaces the definition of divisors has to be localized.

Let $\mathrm{V}$ be a complex vector space of dimension $\mathrm{m}+1>1$. Define $\mathrm{V}_{*}=\mathrm{V}-\{0\}$. Then $\mathbb{C}_{\star}$ operates on $v_{*}$ by multiplication. The quotient space $\mathbb{P}(v)=v_{\star} / \mathbb{C}_{\star}$ is a connected, compact complex manifold of dimension m called the complex projective space of $\mathrm{V}$. The residual map $\mathbb{P}: \mathrm{V}_{*} \rightarrow \mathbb{P}(\mathrm{V})$ is holomorphic. If $\mathrm{A} \subseteq \mathrm{V}$, define $\mathbb{P}(\mathrm{A})=\mathbb{P}\left(\mathrm{A} \cap \mathrm{V}_{\star}\right)=\{\mathbb{P}(z) \mid 0 \neq z \in A\} . \quad$ Let $\mathbb{O}(\mathfrak{b}, \mathrm{V})$ be the set of all holomorphic vector functions $\mathfrak{u}: \mathbb{D}(\mathfrak{b}) \rightarrow \mathrm{v}$. Define $\mathfrak{O}_{\star}(\mathfrak{h}, \mathrm{V})=\mathfrak{G}(\mathfrak{b}, \mathrm{V})-\{0\}$. Two holomorphic vector functions $\mathfrak{u} \in \mathfrak{O}_{*}(\mathfrak{h}, V)$ and $\mathfrak{w} \in \mathscr{O}_{*}(\mathfrak{h}, V)$ are called equivalent $\mathfrak{u} \sim \mathfrak{m}$, if $\mathfrak{u} \wedge \mathfrak{w} \equiv 0$. This defines an equivalence relation on $\mathfrak{U}_{*}(\mathfrak{h}, \mathrm{V})$. An equivalence class $f$ is said to be a meromorphic map from $\mathbb{D}(b)$ into $\mathbb{P}(V)$ and each $\mathfrak{u}$ in $f$ is said to be a representation of $f$. The representation $\mathfrak{u}$ is said to be reduced if for each representation w of $f$ there exists a holomorphic function $g$ such that $w=g u$. Each meromorphic map has a reduced representation and if $\mathfrak{u}$ and $\tilde{\mathfrak{u}}$ are reduced representations, there are holomorphic functions $h$ and $\tilde{h}$ on $\mathbb{D}(\mathfrak{b})$ such that $\mathfrak{u}=\tilde{h} \tilde{\mathfrak{u}}$ and $\tilde{\mathfrak{u}}=\mathrm{h} \mathfrak{u}$. Hence $\mathrm{h} \tilde{\mathrm{h}}=1$ and $\mathrm{h} \in \mathscr{O}^{*}(\mathfrak{h})$ has no zeros. Moreover $\mathfrak{w}=\tilde{\mathrm{gu}} \tilde{\mathfrak{u}}=\mathrm{ghu}$. Hence $\mu_{\mathrm{g}}=\mu_{\mathrm{g}}$. Therefore the non-negative divisor $\mu_{\mu \mathrm{m}}=\mu_{\mathrm{g}}$ is well defined independent of the choice of the reduced representation $u$. Moreover $w$ is reduced if and only if $\mu_{w}=0$.

Let $\mathfrak{u}$ and $\tilde{\mathfrak{u}}$ be reduced representations of the meromorphic map $f$, then the indeterminacy

$$
I_{f}=\{z \in \mathbb{D}(\mathfrak{b}) \mid \mathfrak{u}(z)=0\}=\{z \in \mathbb{D}(\mathfrak{b}) \mid \tilde{\mathfrak{u}}(z)=0\}
$$

is well defined, analytic, with $\operatorname{dim} I_{f} \leq n-2$. If $z \in \mathbb{D}(\mathfrak{b})-I_{f}$, then

$$
f(z)=\mathbb{P}(\mathfrak{u}(z))=\mathbb{P}(\tilde{\mathfrak{u}}(z)) \in \mathbb{P}(V)
$$


is well defined and the map $f: \mathbb{D}(\mathfrak{b})-I_{f} \rightarrow \mathbb{P}(V)$ is holomorphic. If $\mathfrak{w}$ is any representation of $f$ and if $z \in \mathbb{D}(\mathfrak{b})$ with $\mathfrak{w}(z) \neq 0$, then $f(z)=\mathbb{P}(\mathfrak{w}(z))$.

Let $\mathrm{W}$ be a $(p+1)$-dimensional linear subspace of $\mathrm{V}$. The projective space $\mathbb{P}(\mathrm{W})$ is a projective plane of dimension $p$ in $\mathbb{P}(V)$. If $p=m-1$, then $\mathbb{P}(W)$ is $\underline{\text { called }}$ a hyperplane. Let $V *$ be the dual vector space of $V$. Take a $\in \mathbb{P}(V *)$. Then $a=\mathbb{P}(\alpha)$ where $\alpha \in \mathrm{V}_{*}^{*}$ is a linear map $\alpha: \mathrm{V} \rightarrow \mathbb{C}$. Then $E[\mathrm{a}]=\mathbb{P}(\operatorname{ker} \alpha)$ is a hyperplane in $\mathbb{P}(\mathrm{V})$. The map $\mathrm{a} \rightarrow \mathrm{E}[\mathrm{a}]$ is a bijective parameterization of all hyperplanes in $\mathbb{P}(\mathrm{V})$. If $a \in \mathbb{P}\left(V^{*}\right)$ and if $f\left(\mathbb{D}(b)-I_{f}\right) \notin E[a]$, then $f$ is said to be linearly $\underline{\text { non-degenerate }} \underline{\text { for }} a$. If $u$ is a reduced representation of $f$ and if $\alpha \in v_{*}^{*}$ then $\alpha \circ u \neq 0$. The divisor $\mu_{f}^{a}=\mu_{\alpha \circ u}$ depends on a and $f$ only and is called the a-divisor of $f$. The valence function of $f$ for $a$ is defined by

$$
N_{f}(r, q ; a)=N_{\mu_{f}^{a}}^{a}(r, q) .
$$

If $\mathfrak{w}$ is a representation of $f$, there is a holomorphic function $g \neq 0$ with $w=g u$. Hence $\alpha \circ \mathfrak{w}=g \alpha \circ \mathfrak{u}$. By definition $\mu_{\mathfrak{w}}=\mu_{g} \cdot$ Hence

$$
\mu_{\alpha \circ w}=\mu_{w}+\mu_{f}^{a}
$$

Take a positive hermitian form (1): $\mathrm{V} \times \mathrm{V} \rightarrow \mathbb{C}$, called a hermitian product on $\mathrm{V}$. The associated norm is defined by $\|\boldsymbol{z}\|=\sqrt{(\boldsymbol{z} \mid \boldsymbol{z})}$. If $\boldsymbol{x} \subset \mathrm{V}$ and $\boldsymbol{z} \in \mathrm{V}$, we have the Schwarz inequality

$$
|(z \mid x)| \leq\|z\|\|x\|
$$

If $\alpha \in V^{*}$ and $\beta \in V^{*}$, vectors $\mathfrak{a} \in \mathrm{V}$ and $\mathfrak{b} \in \mathrm{V}$ exist uniquely such that

$$
\alpha(z)=(z \mid \mathfrak{a}) \quad \beta(z)=(z \mid \mathfrak{b}) \quad \text { for all } z \in V \text {. }
$$

A dual hermitian product on $V^{*}$ is defined by

$$
(\alpha \mid \beta)=(\mathbf{a} \mid \mathbf{b})
$$


If $\alpha=\beta$, then $\|\alpha\|=\|\mathfrak{a}\|$. Therefore (3.20) and (3.21) imply the Schwarz inequality

$$
|\alpha(z)| \leq\|\alpha\|\|z\|
$$

If $z \in \mathbb{P}(V)$ and $a \in \mathbb{P}\left(V^{*}\right)$, then $z=\mathbb{P}(z)$ and $a=\mathbb{P}(\alpha)$ with $z \in V_{*}$ and $\alpha \in V_{*}^{*}$ The projective distance from $z$ to $E[a]$ is defined by

$$
0 \leq\|z, a\|=\frac{|\alpha(z)|}{\|\alpha\|\|z\|} \leq 1
$$

independent of the choice of the representatives $z$ and $\alpha$.

The compensation function of $f$ for a is defined for $r \in \mathbb{R}^{+}(\mathfrak{b})$ by

$$
0 \leq m_{f}(r, a)=\mathbb{M}\left(\log \frac{1}{\|f, a\|}, \mathfrak{r}\right)=\int_{\mathbb{D}\langle\mathbf{r}\rangle} \log \frac{1}{\|f, a\|} \Omega_{n}<\infty .
$$

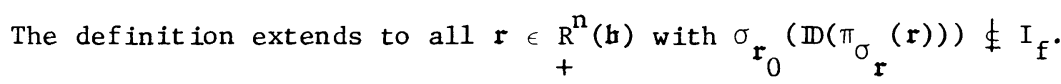

Let $\mathfrak{u}$ be a reduced representation of $f$. Then $\log \|\mathfrak{u}\|$ is a plurisubharmonic function on $\mathbb{D}(\mathbf{b})$. If $\tilde{u}$ is another reduced representation of $f$ a holomorphic function h without zeros exists such that $\tilde{u}=h u$. Then

$$
\log \|\tilde{u}\|=\log \|\mathbf{u}\|+\log |\mathbf{h}|
$$

where $\log |\mathrm{h}|$ is pluriharmonic. Hence $\log \|\tilde{u}\|$ and $\log \|\mathbf{u}\|$ define the same class

$$
\rho(\log \|\tilde{\mathfrak{u}}\|)=\rho(\log \|\mathbf{u}\|)
$$

The characteristic function of $\mathrm{f}$ is defined as the valence function of this class

$$
T_{f}(\mathfrak{r}, \mathfrak{q})=\mathbb{N}(\rho(\log \|\mathbf{u}\|), \mathfrak{r}, \mathfrak{q})
$$

which is

$$
\begin{aligned}
& T_{f}(\mathfrak{r}, \mathfrak{q})=\mathbb{m}(\mathfrak{r}, \log \|\mathbf{u}\|)-(\mathfrak{q}, \log \|\mathbf{u}\|) \\
& T_{f}(\mathfrak{r}, \mathfrak{q})=\int_{\mathbb{D}\langle\mathfrak{r}\rangle} \log \|\mathbf{u}\| \Omega_{n}-\int_{\mathbb{D}\langle\mathfrak{q}\rangle} \log \|\mathbf{u}\| \Omega_{\mathrm{n}} .
\end{aligned}
$$




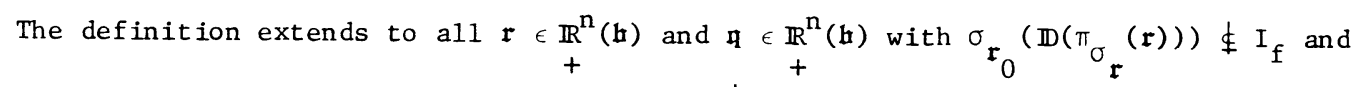
$\sigma_{\mathfrak{\eta}_{0}}\left(\mathbb{D}\left(\pi_{\sigma_{\mathfrak{q}}}(\mathfrak{q})\right)\right) \ddagger I_{\mathfrak{f}} . \quad$ If $\mathfrak{r}, \mathfrak{p}, \mathbf{q}$ belong to $\mathbb{R}^{\prime}(\mathfrak{h})$, then

$$
\begin{aligned}
T_{f}(\mathfrak{r}, \mathfrak{p})+T_{f}(\mathfrak{p}, \mathfrak{p}) & =T_{f}(\mathfrak{r}, \mathfrak{p})=-T_{f}(\mathfrak{p}, \mathfrak{r}) \\
& T_{f}(\mathfrak{r}, \mathfrak{p}) \geq 0 \quad \text { if } 0<\mathfrak{q} \leq \mathfrak{r}<\mathfrak{k} .
\end{aligned}
$$

The function $T_{f}(\mathfrak{r}, \mathfrak{h})$ is logarithmic convex and increasing in $\mathfrak{r}$ and decreasing in $\mathfrak{h}$ and continuous where $0<\mathfrak{q} \leq \mathfrak{r}<\mathfrak{h}$.

Let $\mathfrak{w}$ be a representation of $f$. Take a reduced representation $u$ of $f$. A holomorphic function $g \neq \equiv$ exists uniquely such that $\mathfrak{w}=\mathrm{gu}$. By definition $\mu_{\mathfrak{u}}=\mu_{\mathrm{g}}$. Then

$$
\int_{\mathbb{D}\langle\mathbf{r}\rangle} \log \|\mathbf{w}\| \Omega_{\mathrm{n}}=\int_{\mathbb{D}\langle\mathfrak{r}\rangle} \log \|\mathbf{u}\| \Omega_{\mathrm{n}}+\sum_{\mathbb{D}<\mathfrak{r}>} \log |\mathrm{g}| \Omega_{\mathbf{n}}
$$

Therefore (3.28) and (3.13) imply

$$
\mathrm{T}_{\mathrm{f}}(\mathfrak{r}, \mathfrak{q}\rangle=\sum_{\mathbb{D}\langle\dot{r}} \log \|\mathbf{w}\| \Omega_{n}-\int_{\mathbb{D}\langle\mathfrak{q}\rangle} \log \|\mathbf{w}\| \Omega_{n}-N_{\mu_{\mathfrak{w}}}(\mathfrak{r}, \mathfrak{q})
$$

which generalizes (3.28).

Let $u$ be a reduced representation of $f$. Take $a \in \mathbb{P}(V)$. Assume that $f$ is not linearly degenerate for a. Take $a \in V_{*}^{*}$ with $\mathbb{P}(\alpha)=a$. Take $0<\mathfrak{r}<\mathbf{b}$ and $0<\mathfrak{q}<\mathbf{b}$. Then

$$
\begin{aligned}
& T_{f}(\mathfrak{r}, \mathfrak{q})=\int_{\mathbb{D}\langle\mathfrak{r}\rangle} \log \|\mathbf{u}\| \Omega_{n}-\int_{\mathbb{D}\langle\mathbf{q}\rangle} \log \|\mathbf{u}\| \Omega_{n}
\end{aligned}
$$

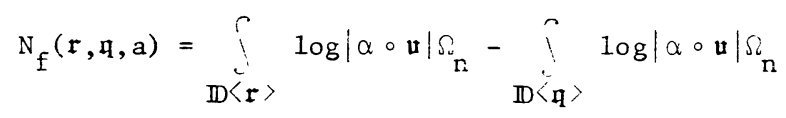

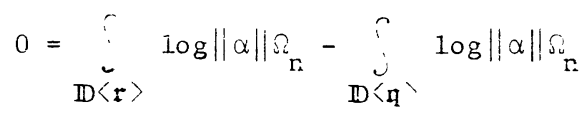




$$
\begin{aligned}
& m_{f}(\mathfrak{r}, a)=\int_{\mathbb{D}\langle\mathfrak{r}\rangle} \log \frac{\|\mathfrak{w}\|\|\alpha\|}{|\alpha \circ \mathfrak{u}|} \Omega_{\mathrm{n}} \\
& \mathrm{m}_{\mathrm{f}}(\mathfrak{q}, a)=\int_{\mathbb{D}\langle\mathfrak{q}\rangle} \log \frac{\|\mathfrak{u}\|\|\alpha\|}{|\alpha \circ \mathfrak{u}|} \Omega_{\mathrm{n}} .
\end{aligned}
$$

Addition and subtraction imply the First Main Theorem

$$
T_{f}(r, \mathfrak{r})=N_{f}(r, \mathfrak{q}, a)+m_{f}(\mathfrak{r}, a)-m_{f}(\mathfrak{q}, a) .
$$

Define $\tau_{V}: V \rightarrow \mathbb{R}$ by $\tau_{V}(z)=\|z\|^{2}$. One and only one positive form $\omega>0$ of bidegree $(1,1)$ exists on $\mathbb{P}(V)$ such that $\mathbb{P} *(\omega)=d^{c} \log \tau$. Here $d=\partial+\bar{\partial}$ and $d^{c}=(i / 4 \pi)(\bar{\partial}-\partial)$. Here $\omega$ is the exterior form of the Fubini-Study Kaehler metric on $\mathbb{P}(\mathrm{V})$. We have

$$
\int_{\mathbb{P}(V)} \omega^{m}=1
$$

Denote the corresponding form on $\mathbb{P}\left(V^{*}\right)$ also by $\omega$. Then

$$
\int_{a \in \mathbb{P}\left(V^{*}\right)} \log \frac{1}{\|z, a\|} \omega^{m}(a)=\frac{1}{2} \sum_{\mu=1}^{m} \frac{1}{\mu} .
$$

An exchange of integration implies

$$
\int_{a \in \mathbb{P}\left(V^{*}\right)} m_{f}(r, a) \omega^{m}(a)=\frac{1}{2} \sum_{\mu=1}^{m} \frac{1}{\mu} .
$$

Integration of the First Main Theorem yields

$$
T_{f}(\mathfrak{r}, \mathfrak{q})=\int_{a \in \mathbb{P}\left(V^{*}\right)} N_{f}(\mathfrak{r}, \mathfrak{q}, a) \omega^{n}(a) .
$$

For $V=\mathbb{C}^{\mathrm{m}+1}$ write $\mathbb{P}_{\mathrm{m}}=\mathbb{P}(\mathrm{V})$ and $\mathbb{P}_{\mathrm{m}}^{*}=\mathbb{P}\left(\mathrm{V}^{*}\right)$. On $\mathbb{C}^{\mathrm{m}+1}$ define $(z \mid w)=z_{1} \bar{w}_{1}+\ldots+z_{m} \bar{w}_{m}$. Identify the compactified plane $\mathbb{C} \cup\{\infty\}$ with $\mathbb{P}_{1}$ by setting 


$$
\mathbb{P}(\mathrm{z}, \mathrm{w})=\frac{\mathrm{z}}{\mathrm{w}} \quad \mathbb{P}(\mathrm{z}, 1)=\mathrm{z} \quad \mathbb{P}(\mathrm{w}, 0)=\mathbb{P}(1,0)=\infty
$$

if $z \in \mathbb{C}$ and $0 \neq w \in \mathbb{C}$. If $\alpha \in\left(\mathbb{C}^{2}\right)^{*}$, define $\alpha(1,0)=\alpha_{1}$ and $\alpha(0,1)=\alpha_{2}$ and identify $\mathbb{P}_{1}^{*}$ with $\mathbb{C} \cup\{\infty\}$ by setting

$$
\mathbb{P}(\alpha)=-\frac{\alpha_{2}}{\alpha_{1}} \text { if } \alpha_{1} \neq 0 \text { and } \mathbb{P}(\alpha)=\infty \text { if } \alpha_{1}=0 \neq \alpha_{2}
$$

If $z \in \mathbb{C}$ and $a \in \mathbb{C}$, then $(z, 1) \in \mathbb{C}^{2}$ with $\mathbb{P}(z, 1)=z$. Also $\alpha \in\left(\mathbb{C}^{2}\right)^{*}$ is defined by setting $\alpha\left(z_{1}, z_{2}\right)=z_{1}-a z_{2}$. Then $\mathbb{P}(\alpha)=a$ and $\alpha(z, 1)=z-a$. Define $\alpha_{\infty}$ by $\alpha_{\infty}(1,0)=0$ and $\alpha_{\infty}(0,1)=1$. Then

$$
\begin{aligned}
& \|z, a\|=\frac{|\alpha(z, 1)|}{\|\alpha\|\|(z, 1)\|}=\frac{|z-a|}{\sqrt{1+|a|^{2}} \sqrt{|z|^{2}+1}} \\
& \|\infty, a\|=\frac{|\alpha(1,0)|}{\|\alpha\|\|(1,0)\|}=\frac{1}{\sqrt{1+|a|^{2}}} \\
& \|z, \infty\|=\frac{\left|\alpha_{\infty}(z, 1)\right|}{\left\|\alpha_{\infty}\right\|\|(z, 1)\|}=\frac{1}{\sqrt{1+|z|^{2}}} \\
& \|\infty, \infty\|=\frac{\left|\alpha_{\infty}(1,0)\right|}{\left\|\alpha_{\infty}\right\|\|(1,0)\|}=0 .
\end{aligned}
$$

Hence the projective distance $\|$,$\| on \mathbb{P}_{1}$ is the chordale distance on the Riemann sphere of diameter 1 in $\mathbb{R}^{3}$. On $\mathbb{C}$ the Fubini-Study form is given by

$$
\omega=\frac{i}{2 \pi} \frac{\mathrm{d} z \wedge \mathrm{d} \bar{z}}{\left(1+|z|^{2}\right)^{2}} \text {. }
$$

A meromorphic function $f$ on $\mathbb{D}(b)$ is the quotient of two holomorphic functions $g$ and $h \neq 0$, such that $h f=g$ and $u=(g, h): \mathbb{D}(h) \rightarrow \mathbb{C}^{2}$ defines a meromorphic map from $\mathbb{D}(b)$ into $\mathbb{P}_{1}$ which is identified with $f$. All meromorphic maps from $\mathbb{D}(b)$ into $\mathbb{P}_{1}$ are obtained this way except the map identical to $\infty$. The representation $u$ is reduced if 
and only if $g$ and $h$ are coprime at every point of $\mathbb{D}(\boldsymbol{b})$. For $a \in \mathbb{C} \subseteq \mathrm{P}_{1}^{*}$ we have

$$
\begin{aligned}
& \mu_{f}^{a}=\mu_{g-a h} \quad \mu_{f}^{\infty}=\mu_{h} \\
& T_{f}(\mathfrak{r}, \mathfrak{q})=\int_{\mathbb{D}\langle\mathbf{r}\rangle} \log \sqrt{|g|^{2}+|h|^{2}} \Omega_{n}-\int_{\mathbb{D}\langle\mathfrak{q}\rangle} \log \sqrt{|g|^{2}+|h|^{2}} \Omega_{n} \\
& m_{f}(r, a)=\int_{\mathbb{D}\langle r\rangle} \log \frac{\sqrt{1+|a|^{2}} \sqrt{1+|f|^{2}}}{|f-a|} \Omega_{n} \\
& \mathrm{~m}_{\mathrm{f}}(\mathfrak{r}, \infty)=\int_{\mathbb{D}\langle\mathfrak{r}\rangle} \log \sqrt{1+|\mathrm{f}|^{2}} \Omega_{\mathrm{n}}
\end{aligned}
$$

Our definition of the value distribution functions corresponds to the AhlforsShimizu definition and is guided by the intrinsic nature of the algebraic and geometric structures which are involved in the given mathematical situation. The classical definitions do not account properly of these features. We would destroy the intrinsic coherence of the theory if we would insist that $q=0$ and that $\mathrm{m}_{\mathrm{f}}(\mathrm{r}, \infty)$ be defined as the integral average of $\log ^{+}|\mathrm{f}|$.

Our definitions recognize the absence of a natural one parameter exhaustion of the polydisc and take advantage of the fact that every holomorphic line bundle on a polydisc is trivial. If the polydisc is replaced by another complex manifold, the definitions have to be localized and holomorphic line bundles become unavoidable, also an appropriate exhaustion has to be chosen.

At present our definitions do not reflect the possible holomorphic slicings of a polydisc and do not present the valence function as an integrated counting furction or the characteristic as an integrated spherical image. These properties will be studied in the next two sections.

4. THE COUNTING FUNCTION.

Take $\mathfrak{b} \in \mathbb{R}^{+}$and $\mathfrak{a} \in \mathbb{D}(\mathfrak{h})$. Let $g \neq 0$ be a holomorphic function on $\mathbb{D}(\mathfrak{b})$. Then there exists uniquely a non-negative integer $\mu_{g}(a)$ called the zero multiplicity of $g$ 
at a such that for each integer $j \geq \mu_{g}(\mathfrak{a})$ there is a homogeneous polynomial $P_{j}$ of degree $j$ such that

$$
g(z)=\sum_{j=\mu_{g}(\mathfrak{a})}^{\infty} P_{j}(z-\mathfrak{a})
$$

for all $z$ in a neighborhood of $\mathfrak{a}$ in $\mathbb{D}(\mathfrak{b})$ and such that $\mathrm{P}_{\mu_{g}}(\mathfrak{a})$ 丰 0 . Obviously $\mu_{g}(\mathfrak{a})=0$ if and only if $g(\mathfrak{a}) \neq 0$. If $h \neq 0$ is a holomorphic function on $\mathbb{D}(\mathfrak{b})$ then

$$
\begin{gathered}
\mu_{g h}(\mathfrak{a})=\mu_{g}(\mathfrak{a})+\mu_{h}(\mathfrak{a}) \\
\mu_{g+h}(\mathfrak{a}) \geq \operatorname{Min}\left(\mu_{g}(\mathfrak{a}), \mu_{h}(\mathfrak{a})\right) .
\end{gathered}
$$

Therefore the map $\mathfrak{O}_{\star}(\mathfrak{h}) \rightarrow \mathbb{Z}_{+}$defined by $g \rightarrow \mu_{g}(\mathfrak{a})$ is a valuation of the $r$ ing $\mathfrak{D}(\mathfrak{b})$. Moreover $\mu_{g h}(\mathfrak{a})=\mu_{g}(\mathfrak{a})$ if and only if $h(\mathfrak{a}) \neq 0$.

Let $\nu$ be a divisor on $\mathbb{D}(\mathfrak{b})$. Then $\nu=\mu_{f}$ where $f \neq 0$ is a meromorphic function on $\mathbb{D}(\mathfrak{b})$. Let $g$ and $h$ be holomorphic functions on $\mathbb{D}(\mathfrak{b})$ which are coprime at every point of $\mathbb{D}(\mathfrak{b})$ such that $h f=g$. Then $(g, h)$ is a reduced representation of the meromorphic map f. Let $\tilde{\mathrm{f}}, \tilde{\mathrm{g}}$ and $\tilde{\mathrm{h}}$ be another choice. A holomorphic function $u \in \mathbb{O} *(\mathfrak{h})$ on $\mathbb{D}(\mathfrak{h})$ without zeros exists such that $\tilde{\mathrm{f}}=$ uf. Then (ug,h) is another reduced representation of $\tilde{\mathrm{f}}$. Hence $\mathrm{v} \in \mathfrak{O}^{*}(\mathfrak{b})$ exists such that $(\tilde{\mathrm{g}}, \tilde{\mathrm{h}})=(\mathrm{vug}, \mathrm{vh})$. Consequent ly

$$
\nu(\mathfrak{a})=\mu_{g}(\mathfrak{a})-\mu_{\mathfrak{h}}(\mathfrak{a})=\mu_{g}(\mathfrak{a})-\mu_{h}(\mathfrak{a}) \in \mathbb{Z}
$$

is well defined independent of the choice of $\mathrm{f}, \mathrm{g}$, and $\mathrm{h}$ and is called the multiplicity of $v$ at $\mathfrak{a}$. The map $\mathbb{D}(\mathfrak{h}) \rightarrow \mathbb{Z}$ defined by $\nu \rightarrow v(\mathfrak{a})$ is a homomorphism: If $\nu_{j} \in \mathbb{D}(\boldsymbol{h})$ and $p \in \mathbb{Z}$, then

$$
\left(p v_{1}+v_{2}\right)(\ddot{u})=p v_{1}(a)+v_{2}(\mathfrak{a}) \quad 0(\mathfrak{a})=0 .
$$

The divisor $\nu$ is non-negative if and only if $\nu(\mathfrak{a}) \geq 0$ for all $\mathfrak{a} \in \mathbb{D}(\mathbf{b})$. The divisor $V$ is the zero divisor if and only if $\nu(\mathfrak{a})=0$ for all a $\in \mathbb{D}(\mathfrak{b})$. Therefore we can identify a divisor $v$ with its multiplicity function $\rightarrow v(a)$ and in fact this is one way of defining divisors on complex manifolds. 
Let $f$ be a meromorphic function on $\mathbb{D}(\mathfrak{b})$. Take $a \in \mathbb{P}_{1}$. If $f \neq$ a, the a-divisor $\mu_{f}^{a}$ is defined. Then $\mu_{f}^{a}(a)$ is called the a-multiplicity of $f$ at $\mathfrak{a}$. If $f$ 丰 0 , the divisor $\mu_{f}=\mu_{f}^{0}-\mu_{f}^{\infty}$ is defined and $\mu_{f}(\mathfrak{a})$ is said to be the multiplicity of $f$ at $\mathfrak{a}$. The function $f \neq 0$ is holomorphic if and only if $\mu_{f}(a) \geq 0$ for $a l l a \in \mathbb{D}(\mathfrak{b})$, and $f$ is holomorphic and without zeros if and only if $\mu_{f}(\mathfrak{a})=0$ for all $\mathfrak{a} \in \mathbb{D}(\mathfrak{b})$. Also $\mu_{1 / f}(\mathfrak{d})=-\mu_{f}(\mathfrak{a})$. If $f_{1} \neq 0$ and $f_{2} \neq=0$ are meromorphic functions on $\mathbb{D}(\mathfrak{b})$ then

$$
\mu_{f_{1} f_{2}}(\mathfrak{a})=\mu_{f_{1}}(\mathfrak{a})+\mu_{f_{2}}(\mathfrak{a})
$$

The map $\mathfrak{K}_{\star}(\mathfrak{h}) \rightarrow \mathbb{Z}$ defined by $\mathbf{f} \rightarrow \mu_{f}(\mathfrak{a})$ is a homomorphism.

Let $\nu$ be a divisor on $\mathbb{D}(\boldsymbol{b})$. The closure in $\mathbb{D}(\mathbf{b})$ of the set of all $z \in \mathbb{D}(\mathbf{b})$ with $\nu(z) \neq 0$ is called the support of $\nu$ and denoted by supp $\nu$. If $\nu=0$ then supp $\nu=\emptyset$. If $\nu \neq 0$, then the support of $\nu$ is an analytic subset of pure dimension $n-1$ of $\mathbb{D}(\boldsymbol{b})$. Let $\mathfrak{R}$ (supp $\nu$ ) be the set of regular points of supp $\nu$. Then the function $\nu$ is constant on the connectivity components of $R(\operatorname{supp} \nu)$. If $\nu \geq 0$, then supp $\nu=\{z \in \mathbb{D}(\mathfrak{b}) \mid \nu(z)>0\}$.

Let $f$ be a meromorphic function on $\mathbb{D}(\mathfrak{b})$ with indeterminacy $I_{f}$. Take a $\in \mathbb{P}_{1}$. Assume that $\mathrm{f} \neq \mathrm{F}$ a. Then

$$
\operatorname{supp} \mu_{f}^{a}=I_{f} \cup\left\{z \in \mathbb{D}(b)-I_{f} \mid f(z)=a\right\}
$$

If $a=0$, then supp $\mu_{f}=\operatorname{supp} \mu_{f}^{0} \cup \operatorname{supp} \mu_{f}^{\infty}$.

Take $\mathrm{p} \in \mathbb{N}$ and $\mathfrak{r} \in \stackrel{\mathbb{R}}{\mathrm{R}}^{\mathrm{p}}$. Let $\phi: \mathbb{D}(\mathfrak{r}) \rightarrow \mathbb{D}(\mathfrak{b})$ be a holomorphic map. Take a divisor $\nu$ on $\mathbb{D}(\mathfrak{b})$ such that $\phi(\mathbb{D}(\mathfrak{r})) \notin$ supp $\nu$. Let $f$ be a meromorphic function on $\mathbb{D}(\mathrm{b})$ such that $\nu=\mu_{\mathrm{f}}$. Then $\mathrm{f} \circ \phi \neq 0$ is a meromorphic function on $\mathbb{D}(\mathfrak{r})$. The pullback divisor

$$
\phi *(\nu)=\mu_{f \circ \phi}
$$

is well defined and independent of the choice of $\mathrm{f}$. If $\nu \geq 0$, then $\phi^{*}(\nu) \geq 0$. If $\mathrm{p}_{j} \in \mathbb{Z}$ and $\nu_{j}$ are divisors on $\mathbb{D}(\mathfrak{b})$ with $\phi(\mathbb{D}(\mathfrak{r})) \notin \operatorname{supp} \nu_{j}$ for $j=1$, 2 , then 
$\phi(\mathbb{D}(r)) \notin \operatorname{supp}\left(p_{1} \nu_{1}+p_{2} \nu_{2}\right)$ and

$$
\phi^{*}\left(p_{1} \nu_{1}+p_{2} \nu_{2}\right)=p_{1} \phi^{*}\left(\nu_{1}\right)+p_{2} \phi^{*}\left(\nu_{2}\right)
$$

For $0 \neq z \in \mathbb{C}^{\mathrm{n}}$ an injective linear map $\ell_{z}: \mathbb{C} \rightarrow \mathbb{C}^{\mathrm{n}}$ is defined by $\ell_{z}(u)=u z$ for al1 $u \in \mathbb{C}$. If $0 \neq z \in \mathbb{D}\langle\mathbf{r}\rangle$ and $0 \leq \mathfrak{r}\left\langle\mathbf{b}\right.$, a largest number $t_{0}(\mathfrak{r})>1$ exists such that $\ell_{\mathfrak{z}}\left(\mathbb{D}\left(t_{0}(\mathfrak{r})\right)\right) \subseteq \mathbb{D}(\mathfrak{r})$. Let $\nu$ be a divisor on $\mathbb{D}(\mathfrak{b})$. Take $\boldsymbol{z} \in \mathbb{D}\langle\mathbf{r}\rangle$, then $\nu$ is said to be restrictable to $z$, if and only if $\ell_{z}\left(\mathbb{D}\left(t_{0}(r)\right)\right) \notin$ supp $\nu$. Let $R_{v}(r)$ be the set of all $z^{\prime} \in \mathbb{D}\langle\mathfrak{r}\rangle$ such that $\nu$ is not restrictable to $z$. Then $R_{\nu}(\mathfrak{r})$ is a thin real analytic subset of $\mathbb{D}\langle\mathfrak{r}\rangle$. For $z \in \mathbb{D}\langle\mathfrak{r}\rangle-\mathrm{R}_{V}(\mathfrak{r})$, the pullback divisor $\nu[z]=\ell_{z}^{*}(\nu)$ is defined. If $u \in \mathbb{C}\left(t_{0}(\mathfrak{r})\right)$, abbreviate $\nu[z, u]=\nu[z](u)$. For $z \in \mathbb{D}\langle\mathbf{r}\rangle-\mathrm{R}_{\nu}(\mathbf{r})$ and $0 \leq t<t_{0}(\boldsymbol{r})$ define the counting function of $\nu$ in the direction $z$ as the finite sum

$$
n_{v}[z, t]=\sum_{u \in \mathbb{D}[t]} v[z, u] .
$$

The function $t \rightarrow n_{v}[z, t]$ is semi-continuous from the right and is of bounded variation on each compact subinterval of $\mathbb{R}\left[0, t_{0}\right)$. There is a thin analytic cone $C_{v}$ with vertex 0 in $\mathbb{C}^{\mathrm{n}}$ such that

$$
\lim _{0<t \rightarrow 0} n_{\nu}[z, t]=n_{\nu}[0, t]=v(0)
$$

for all $0 \neq z \in \mathbb{D}(\mathfrak{b})-C_{\nu}$. Here $\mathbb{D}\langle\mathfrak{r}\rangle \cap C_{\nu}$ is a thin real analytic subset of $\mathbb{D}\langle\boldsymbol{r}\rangle$. If $0 \neq \lambda \in \mathbb{D}\left(t_{0}(\mathfrak{r})\right)$ and $z \in \mathbb{D}\langle\mathfrak{r}\rangle-R_{\nu}(\mathfrak{r})$, then $\lambda \boldsymbol{z} \in \mathbb{D}\langle|\lambda| \mathfrak{r}\rangle-R_{\nu}(|\lambda| \mathfrak{r})$ and

$$
n_{v}[\lambda z, t]=n_{v}[z,|\lambda| t]
$$

If $\nu \geq 0$, the function $t \rightarrow n_{\nu}[z, t]$ is non-negative and increases.

If $\nu_{j}$ are divisors on $\mathbb{D}(\mathfrak{b})$ restrictable to $z \in \mathbb{D}\langle\mathbf{r}\rangle$, and if $p_{j} \in \mathbb{Z}$ for $j=1,2$, then $p_{1} \nu_{1}+p_{2} \nu_{2}$ is restrictable to $z$ and 


$$
\mathrm{n}_{1} \nu_{1}+\mathrm{p}_{2} \nu_{2}[z, \mathrm{t}]=\mathrm{p}_{1} \mathrm{n}_{\nu_{1}}[z, \mathrm{t}]+\mathrm{p}_{2} \mathrm{n}_{\nu_{2}}[z, \mathrm{t}]
$$

Let $\mathrm{f} \neq \mathrm{f} 0$ be a meromorphic function on $\mathbb{D}(\boldsymbol{b})$ such that $\nu=\mu_{\mathrm{f}}$. Take $\boldsymbol{z} \in \mathbb{D}\langle\mathfrak{r}\rangle-\mathrm{R}_{\nu}(\mathfrak{r})$. Then $\nu[z]=\mu_{f \circ \ell_{z}}$. For $0<q<r<t_{0}(\mathfrak{r})$ the Jensen formula and the definition of the valence function for $v[z]$ imply

$$
N_{\nu[z]}(r, q)=\int_{\mathbb{D}\langle r\rangle} \log \left|f \circ l_{z}\right| \Omega_{1}-\int_{\mathbb{D}\langle q\rangle} \log \left|f \circ l_{z}\right| \Omega_{1}=\int_{q}^{r} n_{\nu}[z, t] \frac{d t}{t} .
$$

The function $z \rightarrow N_{\nu[z]}(r, q)$ is continuous on $\mathbb{D}\langle\mathfrak{r}\rangle-C_{\nu}$, hence measurable on $\mathbb{D}\langle\mathbf{r}\rangle$. Also

$$
\frac{N_{\nu[z]}(\theta r, r)}{\log \theta} \rightarrow n_{\nu}[z, r] \text { for } 1<\theta \rightarrow 1
$$

implies that $n_{\nu}[z, r]$ is a measurable function of $z$ on $\mathbb{D}(r)$. By $(2.6) N_{v[z]}(\theta r, r)$ is integrable over $\mathbb{D}\langle\mathfrak{r}\rangle$ if $\mathrm{r}\left\langle\theta \mathbf{r}<\mathrm{t}_{0}(\mathfrak{r})\right.$. If $v \geq 0$ then

$$
n_{v}[z, r] \leq \frac{N_{U[z]}(\theta r, r)}{\log \theta} \text { for } 1<\theta<\frac{t_{C}(r)}{r}
$$

Hence $n_{\mathcal{V}}[\boldsymbol{z}, r]$ is integrable over $\mathbb{D}\langle\mathfrak{r}\rangle$. Since every divisor is the difference of two non-negative divisors, $n_{\nu}[z, r]$ is integrable over $\mathbb{D}(\mathfrak{b})$ for all $\nu \in \mathfrak{d}(\mathfrak{b})$. The counting function $n_{v}(\mathfrak{r})$ of $\nu$ is defined by

$$
n_{v}(\mathfrak{r})=\int_{z \in \mathbb{D}\langle\mathfrak{r}\rangle} n_{\nu}[z, 1] \Omega_{n}(z)
$$

Then

$$
n_{v}(t r)=\sum_{z \in \mathbb{D}\langle\mathfrak{r}\rangle} n_{v}[z, t] \Omega_{n}(z) .
$$

If $0<q<r<t_{0}(\mathfrak{r}),(2.6)$ implies 


$$
\begin{aligned}
N_{v}(r r, q r) & =\int_{z \in \mathbb{D}\langle\mathfrak{r}\rangle} N_{v[z]}(r, q) \Omega_{n}(z) \\
& =\int_{z \in \mathbb{D}\langle\mathfrak{r}\rangle} \int_{q}^{r} n_{v}[z, t] \frac{d t}{t} \Omega_{n}(z)=\int_{q}^{r} n_{v}(t r) \frac{d t}{t} .
\end{aligned}
$$

If $\mathrm{p}_{j} \in \mathbb{Z}$ and $\nu_{j} \in \mathbb{D}(\mathbf{b})$ for $j=1,2$, then

$$
\mathrm{n}_{\mathrm{p}_{1} \nu_{1}+\mathrm{p}_{2} \nu_{2}}(\boldsymbol{r})=\mathrm{p}_{1} \mathrm{n}_{\nu_{1}}(\boldsymbol{r})+\mathrm{p}_{2} \mathrm{n}_{\nu_{2}}(\boldsymbol{r})
$$

If $v \geq 0$, then $n_{v}(r) \geq 0$ and $n_{v}(t r)$ increases in $t$.

PROPOSITION 4.1. Take $\mathfrak{b} \in \stackrel{+}{\mathbb{R}}^{n}$. Let $\nu$ be a divisor on $\mathbb{D}(\mathfrak{b})$ such that supp $\nu \cap \mathbb{D}\langle t \boldsymbol{h}\rangle=\emptyset$ for all $t \in \mathbb{R}[0,1)$. Then $\nu$ is the zero divisor.

PROOF. W.1.o.g. we can assume that $v \geq 0$. Then $0 \notin \operatorname{supp} v$. If $0<\mathbf{r}<1$, then $v[\mathbf{r b}]=0$. Hence $n_{v}[\mathbf{r h}, t]=0$ for $0 \leq t \leq 1$. By (4.17) we have $n_{v}(r \mathbf{r})=0$ for a11 $0<\mathrm{r}<1$. Since $0 \$$ supp $\nu$, we are permitted to take $q=0$ in $(4.19)$ and obtain $N_{V}(r h, 0)=0$ for a11 $0 \leq r<1$

Assume that there exists $z_{0} \in$ supp $v$ such that $\mathfrak{r}=\left|\boldsymbol{z}_{0}\right|>0$. Then $\mathbf{r}<\mathbf{b}$. Take $1<\mathrm{p}<\mathrm{q}$ such that $\mathrm{qr}<\mathrm{b}$ and such that $\mathrm{uz}_{0} \& \operatorname{supp} \nu$ if $\mathrm{p} \leq|\mathrm{u}| \leq \mathrm{q}$. An open connected neighborhood $U$ of $z_{0}$ in $\mathbb{D}\langle\mathfrak{r}\rangle$ exists such that $u z \notin$ supp $\nu$ for all $u \in \mathbb{C}$ with $\mathrm{p} \leq|\mathrm{u}| \leq \mathrm{q}$ and $z \in \mathrm{U}$. Rouche's theorem implies

$$
n_{v}[z, t]=n_{v}\left[z_{0}, t\right] \geq 1
$$

for all $z \in U$ and $t \in \mathbb{R}[p, q]$. Hence $n_{V}(t r)>0$ for $p \leq t \leq q$, which implies $N_{V}(q r, 0)>0$ by (4.19). A number $s \in \mathbb{R}(0,1)$ exists such that $q r<s h$. Then

$$
0<N_{V}(q r, 0) \leq N_{V}(s h, 0)=0
$$

which is a contradiction. Hence $z \in \operatorname{supp} v$ at most if a coordinate of $z$ is zero. Since $0 \notin \operatorname{supp} \nu$ we conclude supp $\nu=\emptyset ;$ q.e.d.

By the same procedures as in Lemma 10.4 and Lemma 10.5 of [2], the following result can be proved: 


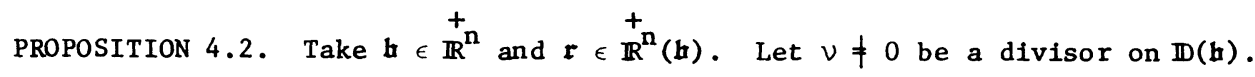
Then $A=\operatorname{supp} \nu$ is an analytic subset of pure dimension $n-1$ in $\mathbb{D}(\mathbf{b})$. Define $A_{*}=A-\{0\}$. Let $A^{0}$ be the set of all $z \in A_{\star}$ such that the restriction $\mathbb{P}: A_{*} \rightarrow \mathbb{P}_{n-1}$ is locally biholomorphic. Then $A^{0}$ is either empty or a complex manifold of dimension $n-1$. The complement $A_{*}-A^{0}$ is analytic in $A_{\star}$ Also $A^{0} \cap \Delta(r)$ is either empty or an $(n-1)$-dimensional real analytic manifold which can be oriented such that $\xi_{n}$ defines a positive measure on $A^{0} \cap \Delta(r)$. We have

$$
\mathrm{n}_{\nu}(\mathbf{r})=\int_{\mathrm{A}^{0} \cap \Delta(\mathbf{r})} v \xi_{\mathrm{n}}+v(0)
$$

Take $b \in \stackrel{+}{\mathbb{R}^{n}}$ and let $\nu$ be a divisor on $\mathbb{D}(\boldsymbol{b})$. Take $p \in \mathbb{N}[1, n)$ and define $\tilde{p}=n-p$. Take $\tau \in \mathbb{C}(p, n)$. Then $\tilde{\tau} \in \mathbb{C}(\tilde{p}, n)$. Take any $z \in \mathbb{D}(\pi \tilde{\tau}(b))$. Then $\tau_{\boldsymbol{z}}: \mathbb{D}\left(\pi_{\tau}(\mathfrak{b})\right) \rightarrow \mathbb{D}(\mathfrak{b})$ is an injective holomorphic map. We say that $\nu$ is

restrictable to $z$ by $\tau$ if

$$
\tau_{\boldsymbol{Z}}\left(\mathbb{D}\left(\pi_{\tau}(\boldsymbol{b})\right)\right) \notin \operatorname{supp} v \text {. }
$$

Let $R_{\nu}(\tau)$ be the set of all $z \in \mathbb{D}(\pi \tilde{\tau}(\mathbf{h}))$ such that $\nu$ is not restrictable to $z$ by $\tau$. If $z \in \mathbb{D}(\pi \tilde{\tau}(b))-R_{\nu}(\tau)$, the pullback divisor

$$
v[\tau, z]=\tau_{z}^{*}(\nu)
$$

exists.

PROPOSITION 4.3. Take $\mathfrak{r} \in \stackrel{+}{\mathbb{R}}^{n}(\mathfrak{b})$ and $\mathfrak{q} \in \stackrel{+}{\mathbb{R}^{n}}(\mathfrak{b})$. Assume that $\mathfrak{p}=\pi \tilde{\tau}(\mathfrak{r})=\pi \tilde{\tau}(\mathfrak{q})$. Then

$$
N_{\nu}(\boldsymbol{r}, \mathfrak{q})=\int_{z \in \mathbb{D}\langle\mathfrak{p}\rangle} N_{V[\tau, z]}\left(\pi_{\tau}(\mathfrak{r}), \pi_{\tau}(\mathfrak{q})\right) \underset{\mathrm{p}}{\Omega_{\sim}(z)}
$$

PROOF. A meromorphic function $f \neq 0$ exists on $\mathbb{D}(\boldsymbol{b})$ such that $\mu_{f}=\nu$. By (2.22) we have 


$$
\begin{aligned}
& N_{v}(\mathfrak{r}, \mathfrak{q})=\int_{\mathbb{D}\langle\mathfrak{r}\rangle} \log |f| \Omega_{n}-\int_{\mathbb{D}\langle\mathfrak{p}\rangle} \log |f| \Omega_{n}=\int_{z \in \mathbb{D}\langle\mathfrak{p}\rangle}\left(\int_{\mathbb{D}\left\langle\pi_{\tau}(\mathfrak{r})\right\rangle} \log \left|f \circ \tau_{\mathfrak{z}}\right| \Omega_{\mathrm{p}}\right) \Omega_{\mathbf{p}}(\boldsymbol{z}) \\
& -\int_{z \in \mathbb{D}\langle\boldsymbol{p}\rangle}\left(\int_{\mathbb{D}\left\langle\pi_{\tau}(\mathfrak{q})\right\rangle} \log \left|\mathbf{f} \circ \tau_{\boldsymbol{z}}\right| \Omega_{\mathrm{p}}\right) \Omega_{\mathbf{p}}(\boldsymbol{z})=\int_{\boldsymbol{z} \in \mathbb{D}\langle\mathfrak{p}\rangle} N_{\nu[\tau, \boldsymbol{z}]}\left(\pi_{\tau}(\boldsymbol{r}), \pi_{\tau}(\mathfrak{p})\right) \Omega_{\mathbf{p}}(\boldsymbol{z}) \\
& \text { If } \pi_{\tau}(\mathfrak{q})=q \pi_{\tau}(\mathfrak{r}) \text { with } 0<q<1 \text {, then (4.19) implies } \\
& N_{\nu[\tau, z]}\left(\pi_{\tau}(r), q \pi_{\tau}(r)\right)=\int_{q}^{l} n_{\nu[\tau, z]}\left(t \pi_{\tau}(r)\right) \frac{d t}{t}
\end{aligned}
$$

For $x \in \pi_{\tau}(b)$ define

$$
\mathrm{n}_{\nu, \tau}(\mathfrak{p}, \boldsymbol{x})=\int_{z \in \mathbb{D}\langle\mathfrak{p}\rangle} \mathrm{n}_{\nu[\tau, \boldsymbol{z}]}(\boldsymbol{x}) \Omega_{\mathbf{p}}
$$

We have

$$
N_{\nu}(r, q)=\int_{q}^{1} n_{\nu, \tau}\left(\mathfrak{p}, t \pi_{\tau}(r)\right) \frac{d t}{t}
$$

provided $\pi_{\tilde{\tau}}(\mathfrak{r})=\pi_{\tilde{\tau}}(\mathfrak{q})=\mathfrak{p}$ and $\pi_{\tau}(\mathfrak{q})=q \pi_{\tau}(\mathfrak{r})$ with $0<q<1$.

If $\tilde{\tau}_{0}\left(\mathbb{D}\left(\pi \tilde{\tau}^{(b)}\right)\right) \notin \operatorname{supp} \nu$, we are permitted to use $q=0$

$$
N_{\nu}(\mathfrak{r}, \mathfrak{q})=\int_{0}^{1} n_{\nu, \tau}\left(\mathfrak{p}, t \pi_{\tau}(\mathfrak{r})\right) \frac{d t}{t} .
$$

If we abbreviate $\mu=\tilde{\tau}_{0}^{*}(\nu)$, then we have $N_{\nu}(\mathfrak{r}, \mathfrak{q})=N_{\nu}(\mathfrak{r}, 0)-N_{\mu}(\mathfrak{p}, 0)$ which leads us to a generalization of formula 4.2 .3 by Ronkin [1]

$$
N_{\nu}(r, 0)-N_{\mu}(\mathfrak{p}, 0)=\int_{0}^{1} n_{\nu, \tau}\left(\mathfrak{p}, t \pi_{\tau}(r)\right) \frac{d t}{t} .
$$

Take $\mathrm{p}=1$ and identify $\tau:\{1\} \rightarrow \mathbb{N}[1, \mathrm{n}]$ with $\tau(1) \in \mathbb{N}[1, \mathrm{n}]$. Then $\pi_{\tau}(\mathbf{r})=\mathbf{r}_{\tau}$ is the $\tau^{\text {st }}$ coordinate and $q_{\tau}=q r_{\tau}$. Hence

$$
\int_{q}^{1} n_{\nu, \tau}\left(\mathfrak{p}, t \pi_{\tau}(\mathfrak{r})\right) \frac{d t}{t}=\int_{q_{\tau}}^{r_{\tau}} n_{\nu, \tau}(\mathfrak{p}, t) \frac{d t}{t} .
$$

If $r=\left(r_{1}, \ldots, r_{n}\right)$ and $\boldsymbol{q}=\left(r_{1}, \ldots, r_{\tau-1}, q_{\tau}, r_{\tau+1}, \ldots, r_{n}\right)$, then 


$$
N_{\nu}(\mathfrak{r}, \mathfrak{q})=\int_{q_{\tau}}^{r_{\tau}} n_{\nu, \tau}(\mathfrak{p}, t) \frac{d t}{t}
$$

where $\mathfrak{p}=\left(\mathrm{r}_{1}, \ldots, \mathrm{r}_{\tau-1}, \mathrm{r}_{\tau+1}, \ldots, \mathrm{r}_{\mathrm{n}}\right)$ and

$$
\begin{gathered}
\mathrm{n}_{\nu, \tau}(\mathfrak{p}, t)=\int_{z \in \mathbb{D}\langle\mathfrak{p}\rangle} \mathrm{n}_{\nu[\tau, z]}(t) \Omega_{\mathrm{n}-1}(z) \\
\mathrm{n}_{\nu[\tau, z]}(\mathrm{t})=\sum_{u \in \mathbb{D}[t]} \nu[\tau, z, u] .
\end{gathered}
$$

If $0<\mathfrak{q}<\mathfrak{r}<\mathfrak{b}$ are arbitrarily picked, we can represent $N_{\mathcal{V}}(\mathfrak{r}, \mathfrak{q})$ as an integral sum. Define

$$
\begin{aligned}
& \mathfrak{r}_{\tau}=\left(q_{1}, \ldots, q_{\tau}, r_{\tau+1}, \ldots, r_{n}\right) \\
& \mathfrak{p}_{\tau}=\left(q_{1}, \ldots, q_{\tau-1}, r_{\tau+1}, \ldots, r_{n}\right) .
\end{aligned}
$$

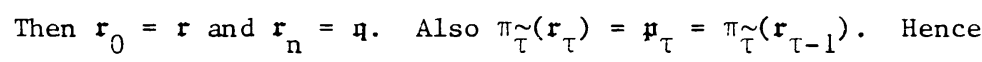

$$
N_{\nu}\left(\mathfrak{r}_{\tau-1}, \mathfrak{r}_{\tau}\right)=\int_{q_{\tau}}^{r_{\tau}} n_{\nu, \tau}\left(\mathfrak{p}_{\tau}, t\right) \frac{d t}{t} .
$$

By addition

$$
N_{\nu}(\mathfrak{r}, \mathfrak{r})=\sum_{\tau=1}^{n} \int_{q_{\tau}}^{r} n_{\nu, \tau}\left(\mathfrak{p}_{\tau}, t\right) \frac{d t}{t} .
$$

\section{THE SPHERICAL IMAGE AND THE CHARACTERISTIC.}

First let us consider the 1 -dimensional case. Take $b \in \stackrel{+}{\mathbb{R}}$. Let $\mathrm{V}$ be a complex vector space of dimension $m+1$ with a hermitian product. Let $\omega$ be the associated Fubini-Study form on $\mathbb{P}(v)$. Take a meromorphic map $f: \mathbb{D}(b) \rightarrow \mathbb{P}(V)$. Since $\mathbb{D}(b)$ has dimension 1 , the map $f$ is holomorphic. The spherical image of $f$ is defined by

$$
A_{f}(t)=\int_{\mathbb{D}(t)} f *(\omega) \quad \forall t \in \mathbb{R}[0, b) .
$$


The function $A_{f}$ increases and is non-negative. Also $A_{f}(t)>0$ if and only if $f$ is not constant and $0<\mathrm{t}<\mathrm{b}$. If $0 \leq \mathrm{q} \leq \mathrm{r}<\mathrm{b}$, the characteristic is given by

$$
T_{f}(r, q)=\int_{q}^{r} A_{f}(t) \frac{d t}{t} .
$$

The identity (5.2) is easily derived from the definition (3.28) in applying Stokes Theo rem twice.

Take $\mathfrak{h} \in \stackrel{+}{\mathbb{R}}^{n}$. Let $f: \mathbb{D}(\mathfrak{h}) \rightarrow \mathbb{P}(V)$ be a meromorphic map. Let $I_{f}$ be the indeterminacy of $\mathrm{f}$. Let $\mathfrak{u}: \mathbb{D}(\mathbf{b}) \rightarrow \mathrm{V}$ be a reduced representation. If $\mathrm{M}$ is a connected complex manifold and if $\phi: M \rightarrow \mathbb{D}(\boldsymbol{h})$ is a holomorphic map such that $\phi(M) \ddagger I_{f}$, then $f \circ \phi$ is a meromorphic function on $M$, and $\boldsymbol{u} \circ \phi$ is a representation which may not be reduced. In order to counterbalance this effect, we have to introduce an indeterminacy multiplicity ${ }_{\mathrm{f}}(\mathfrak{a})$ for each $\mathfrak{a} \in \mathbb{D}(\mathfrak{b})$. There is an integer $v \geq 0$ and for each integer $j \geq v$ a homogeneous vector polynomial $\mathfrak{u}_{j}: \mathbb{C}^{\mathrm{n}} \rightarrow \mathbb{C}$ of degree $\mathrm{j}$ such that $\mathbf{u}_{v} \neq \mathrm{F} 0$ and such that

$$
\mathbf{u}(z)=\sum_{j=v}^{\infty} \mathbf{u}_{j}(z-\mathbf{a})
$$

for all $z$ in an open neighborhood $U$ of a in $\mathbb{D}(\mathfrak{b})$. Define $\imath_{f}(\mathfrak{a})=v$. Obviously $l_{f}(\mathfrak{a})>0$ if and only if $\mathfrak{a} \in I_{f} \cdot$ If $G \neq \emptyset$ is an open, connected subset of $\mathbb{C}$, if $\phi: G \rightarrow \mathbb{D}(\mathfrak{b})$ is holomorphic such that $\phi(G) \notin I_{f}$, then $\mathfrak{u} \circ \phi \neq 0$ and $\mu_{\boldsymbol{u}} \circ \phi(\boldsymbol{x}) \geq \mathrm{I}_{\mathrm{f}}(\phi(\boldsymbol{x}))$. Take $\mathfrak{r} \in \stackrel{+}{\mathbb{R}}^{\mathrm{n}}(\mathbf{r})$ and $z \in \mathbb{D}\langle\mathfrak{r}\rangle$. The meromorphic map $f$ is said to be restrictable to $z$ if and only if $\ell_{z}\left(\mathbb{D}\left(t_{0}(\mathfrak{r})\right)\right) \notin I_{f}$. The set $R_{f}(\mathfrak{r})$ of all $z \in \mathbb{D}\langle\mathfrak{r}\rangle$ such that $f$ is not restrictable to $\boldsymbol{z}$, is a thin real analytic subset of $\mathbb{D}\langle\mathbf{r}\rangle$. For $\boldsymbol{z} \in \mathbb{D}\langle\mathbf{r}\rangle-R_{f}(\mathfrak{r})$, a meromorphic map

$$
\mathrm{f}[\mathbf{z}]=\mathrm{f} \circ \ell_{z}: \mathbb{D}\left(t_{0}(r)\right) \rightarrow \mathbb{P}(V)
$$

is defined. For $0 \leq t<t_{0}(\boldsymbol{r})$ the spherical image of $f$ in the direction $z$ is defined by 


$$
A_{f}[z, t]=A_{f[z]}(t)+l_{f}(0)=\int_{D(t)} f[z] *(\omega)+z_{f}(0) \geq 0
$$

If $0 \neq u t \in \mathbb{D}\left(t_{0}(r)\right)$, then $u z \in \mathbb{D}\langle|u| r\rangle-R_{f}(|u| r)$ and

$$
A_{f}[u z, t]=A_{f}[z,|u| t] .
$$

The function $t \rightarrow A_{f}[z, t]$ increases. For $0<q<r<t_{0}(r)$ define the characteristic of $\mathrm{f}$ in the direction $z$ by

$$
T_{f}[z, r, q]=T_{f[z]}(r, q)+l_{f}(0) \log \frac{r}{q}=\int_{q}^{r} A_{f}[z, t] \frac{d t}{t} \geq 0 .
$$

Let $\mathfrak{w}: \mathbb{D}(\mathfrak{b}) \rightarrow \mathrm{V}$ be a reduced representation of $f$. For $\boldsymbol{z} \in \mathbb{D}\langle\boldsymbol{r}\rangle-\mathrm{R}_{\mathbf{f}}(\mathbf{r})$ the vector function $\mathfrak{u} \circ \ell_{\mathfrak{z}} \neq 0$ is a representation of $f[z]$ on $D\left(t_{0}(\mathfrak{r})\right)$. Let $\mu_{\mathfrak{u} \circ l_{z}}$ be the divisor of $\mathfrak{u} \circ \ell_{z}$ on $\mathbb{C}$. Define the divisor $\mu_{\mathfrak{u}}[z] \geq 0$ on $\mathbb{D}\left(t_{0}(\mathfrak{r})\right)$ by

$$
\mu_{u}[z, u]= \begin{cases}\mu_{u} \circ l_{z}(u) \geq 0 & \text { if } 0 \neq u \in \mathbb{D}\left(t_{0}(r)\right) \\ \mu_{u} \circ l_{z}(0)-l_{f}(0) \geq 0 & \text { if } u=0 .\end{cases}
$$

If $0 \neq u \in \mathbb{D}\left(t_{0}(r)\right)$, then $\mu_{u}[z, u]>0$ if and only if $\ell_{z}(u)=u z \in I_{f}$. If $u=0$, then $\mu_{\mathfrak{u}}[z, u]>0$ if in (5.3) with $a=0$ we have $u_{v}(z)=0$. Since $\operatorname{dim} I_{f} \leq n-2$ and $\mathfrak{u}_{\nu} \neq 0$, we see that $\mu_{\mathfrak{u}} \equiv 0$ for almost all $\boldsymbol{z} \in \mathbb{D}\langle\mathbf{r}\rangle-\mathrm{R}_{\mathbf{f}}(\mathbf{r})$. By (3.31) and (5.7) we obtain

$$
T_{f}[z, r, q]=\int_{\mathbb{D}\langle r\rangle} \log \left\|\mathbf{u} \circ \ell_{z}\right\| \Omega_{1}-\int_{\mathbb{D}\langle q\rangle} \log \left\|\mathbf{u} \circ \ell_{z}\right\| \Omega_{1}-N_{\mu_{u}[z]}(r, q) .
$$

Since $N_{\mu_{\mathfrak{u}}[z]}=0$ for almost all $z$ in $\mathbb{D}\langle\mathfrak{r}\rangle$, the function $T_{f}[z, r, q]$ is integrable over $\mathbb{D}\langle\mathbf{r}\rangle$ and (2.6), (3.27) and (5.9) imply

$$
\mathrm{T}_{\mathrm{f}}(\mathrm{r} \boldsymbol{r}, q \mathfrak{q})=\int_{z \in \mathbb{D}\langle\mathfrak{r}\rangle} \mathrm{T}_{\mathrm{f}}[\boldsymbol{z}, \mathrm{r}, \mathrm{q}] \Omega_{\mathrm{n}}(\boldsymbol{z}) .
$$


If $z \in \mathbb{D}\langle\mathbf{r}\rangle-\mathrm{R}_{\mathrm{f}}(\boldsymbol{r})$ and if $0<t<\theta t<t_{0}(\boldsymbol{r})$, then

$$
A_{f}[z, t] \leq \frac{T_{f}[z, \theta t, t]}{\log \theta} \rightarrow A_{f}[z, t] \quad \text { for } \theta \rightarrow 1
$$

Therefore $A_{f}[z, t]$ is integrable over $\mathbb{D}\langle r\rangle$. The spherical image of $f$ is defined by

$$
A_{f}(r)=\int_{z \in \mathbb{D}\langle\mathbf{r}\rangle} A_{f}[z, 1] \Omega_{n} .
$$

The identity (5.6) implies

$$
A_{f}(t r)=\int_{z \in \mathbb{D}\langle r\rangle} A_{f}[z, t] \Omega_{n} \text { for } 0 \leq t<t_{0}(r) .
$$

From (5.7), (5.10) and (5.13) we obtain

$$
T_{f}(r r, q r)=\int_{q}^{r} A_{f}(t r) \frac{d t}{t}
$$

If $0<q<\mathbf{r}<\infty$ and $x \in \stackrel{+}{\mathbb{R}}$ define

$$
L(x, r, q)=\log ^{+} \frac{r}{x}-\log ^{+} \frac{q}{x}=\left\{\begin{array}{cl}
0 & \text { if } x \geq r \\
\log \frac{r}{x} & \text { if } q \leq x \leq r \\
\log \frac{r}{q} & \text { if } 0<x \leq q .
\end{array}\right.
$$

PROPOSITION 5.1. If $r \in \stackrel{+}{\mathbb{R}}^{n}(\mathfrak{b})$ and $0<\mathrm{q}<\mathbf{r}<\mathrm{t}_{0}(\mathbf{r})$, then

$$
\begin{gathered}
A_{f}(r)=\int_{\Delta(r)} f *(\omega) \wedge \xi_{n}+l_{f}(0) \\
T_{f}(r r, q r)= \\
\int_{z \in \Delta(r)} L\left(\frac{\|z\|}{\|r\|}, r, q\right) f *(\omega) \wedge \xi_{n}+l_{f}(0) \log \frac{r}{q} .
\end{gathered}
$$

PROOF. If $z \in \mathbb{D}\langle\mathfrak{r}\rangle-\mathrm{R}_{\mathbf{f}}(\mathfrak{r})$, then

$$
f[z] *(\omega)=\left(f \circ \ell_{z}\right) *(\omega)=l_{z}^{*}(f *(\omega)) .
$$


Therefore Lemma 2.1 implies

$$
\begin{aligned}
A_{f}(\mathfrak{r}) & =\int_{z \in \mathbb{D}\langle\mathfrak{r}\rangle}\left(\int_{\mathbb{D}} f[z] *(\omega)+\imath_{f}(0)\right) \Omega_{n}(z) \\
& =\int_{z \in \mathbb{D}\langle\mathfrak{r}\rangle} \int_{\mathbb{D}} e_{z}^{*}(f *(\omega)) \Omega_{n}(z)+l_{f}(0)=\int_{\Delta(\mathfrak{r})} f *(\omega) \wedge \xi_{n}+\imath_{f}(0) .
\end{aligned}
$$

If $z \in \Delta\left(t_{0}(\mathfrak{r}) \mathfrak{r}\right)$, then $|z|=u(z) r$ with $0<u(z)<t_{0}(\mathfrak{r})$. Moreover $\|z\|=u(z)\|r\|$. If $0<t<t_{0}(\mathfrak{r})$ then $z \in \Delta(t r)$ if and only if $0<u(z)<t$. Define $x(x, t)=1$ if $q \leq x \leq t \leq r$ and $x(x, t)=0$ if $q \leq t<x \leq r$. Then

$$
\begin{aligned}
T_{f}(r \mathfrak{r}, q r) & =\int_{q}^{r}\left(\int_{\Delta(t r)} f *(\omega) \wedge \xi_{n}+i_{f}(0)\right) \frac{d t}{t} \\
& =\int_{q}^{r}\left(\int_{\Delta(r r)-\Delta(q r)} x(u(z), t) f *(\omega) \wedge \xi_{n}\right) \frac{d t}{t}+A_{f}(q r) \log \frac{r}{q} \\
& =\int_{\Delta(r r)-\Delta(q r)} \int_{q}^{r} x(u(z), t) \frac{d t}{t} f *(\omega) \wedge \xi_{n}+A_{f}(q r) \log \frac{r}{q} \\
& =\int_{\Delta(r r)-\Delta(q r)} \log \frac{r}{u(z)} f *(\omega) \wedge \xi_{n}+\int_{\Delta(q r)} \log \frac{r}{q} f *(\omega) \wedge \xi_{n}+l_{f}(0) \log \frac{r}{q} \\
& =\int_{\wedge(r r)} L(u(z), r, q) f *(\omega) \wedge \xi_{n}+\imath_{f}(0) \log \frac{r}{q}
\end{aligned}
$$

Take a $\in \mathbb{P}\left(V^{*}\right)$ and assume that $f$ is not linearly degenerate for a. Take $\mathfrak{r} \in \mathbb{R}^{\mathrm{n}}(\mathfrak{r})$ and $\boldsymbol{z} \in \mathbb{D}\langle\mathfrak{r}\rangle-\mathrm{R}_{\mathrm{f}}(\mathfrak{r})$. If $\mu_{\mathrm{f}}^{\mathrm{a}}$ is restrictable to $\boldsymbol{z}$, then

$$
\mathrm{m}_{\mathrm{f}[z]}(\mathrm{r}, \mathrm{a})=\int_{\mathbb{D}\langle\mathrm{r}\rangle} \log \frac{1}{\left\|\mathrm{f} \circ \ell_{z}, a\right\|} \Omega_{1}
$$

is defined for $0<\mathrm{r}<\mathrm{t}_{0}(\mathfrak{r})$. If $0 \neq u \in \mathbb{C}$ with $|u| t<t_{0}(\mathfrak{r})$, then

$$
m_{f[u z]}(r, a)=m_{f[z]}(|u| r, a) .
$$

Al so (2.6) implies

$$
m_{f}(r, a)=\int_{\mathbb{D}\langle\mathfrak{r}\rangle} \log \frac{1}{\|f, a\|} \Omega_{n}=\int_{z \in \mathbb{D}\langle\mathbf{r}\rangle} \int_{\mathbb{D}\langle 1\rangle} \log \frac{1}{\left\|f \circ \ell_{z}, a\right\|} \Omega_{1} \Omega_{n}(z)
$$




$$
\begin{aligned}
& \mathrm{m}_{\mathrm{f}}(\mathrm{r}, \mathrm{a})=\int_{z \in \mathbb{D}\langle\mathfrak{r}\rangle} \mathrm{m}_{\mathrm{f}[z]}(1, \mathrm{a}) \Omega_{\mathrm{n}}(z) \\
& \mathrm{m}_{\mathrm{f}}(\mathrm{r} r, \mathrm{a})=\int_{z \in \mathbb{D}\langle\mathfrak{r}\rangle} \mathrm{m}_{\mathrm{f}[\boldsymbol{z}]}(\mathrm{r}, \mathrm{a}) \Omega_{\mathrm{n}}(z) .
\end{aligned}
$$

Now, we will slice the characteristic parallel to the coordinate planes. Here we encounter the difficulty, that the pullback of a reduced representation of a meromorphic map may not be a reduced representation anymore. We will show that the problem does not occur for "almost all slices". Some preparations are needed. For the technical apparatus we refer to Andreotti-Stol1 [1] $\S 1$.

LEMMA 5.2. Take $\mathbf{b} \in \mathbb{R}^{+}$and $\mathfrak{r} \in \stackrel{+}{\mathbb{R}}^{n}(\mathbf{b})$. Let $\mathrm{A}$ be an almost thin subset of $\mathbb{D}(\mathbf{b})$. Then $A \cap \mathbb{D}\langle\mathbf{r}\rangle$ has measure zero on $\mathbb{D}\langle\mathfrak{r}\rangle$.

PROOF. By definition there is a sequence $\left\{A_{\nu}\right\}_{\nu \in \mathbb{N}}$ of thin subsets of $\mathbb{D}(\mathbf{b})$ such that $A=U_{V=1}^{\infty} A_{V^{\prime}}$ For each $p \in A_{\nu}$ there exists an open neighborhood $U_{V}(p)$ of $p$ in $\mathbb{D}(b)$ and analytic subset $B_{V}(p)$ of $U_{V}(p)$ such that $A_{V} \cap U_{V}(p) \subseteq B_{V}(p)$ and such that $\operatorname{dim} \mathrm{B}_{\nu}(\mathrm{p})<\mathrm{n}$. Hence $\mathrm{B}_{\nu}(\mathrm{p}) \cap \mathbb{D}\langle\mathbf{r}\rangle$ has measure zero on $\mathbb{D}\langle\mathbf{r}\rangle$. Consequently, $A_{V} \cap U_{V}(p) \cap \mathbb{D}\langle\mathfrak{r}\rangle$ has measure zero on $\mathbb{D}\langle\mathbf{r}\rangle$. A countable union of these neighborhoods $U_{V}(p)$ covers $A_{V} \cdot$ Hence $A_{V} \cap \mathbb{D}\langle\mathfrak{r}\rangle$ has measure zero on $\mathbb{D}\langle\mathfrak{r}\rangle$ for each $\nu \in \mathbb{N}$. Therefore $A \cap \mathbb{D}\langle\mathfrak{r}\rangle$ has measure zero on $\mathbb{D}\langle\mathfrak{r}\rangle$; q.e.d.

Take $\mathfrak{h} \in \stackrel{+}{\mathbb{R}^{n}}$. Let $\mathbf{f}: \mathbb{D}(\mathfrak{h}) \rightarrow \mathbb{P}(V)$ be a meromorphic map. Let $\mathbf{u}: \mathbb{D}(\mathbf{h}) \rightarrow \mathrm{V}$ be a reduced representation of $f$. Take $p \in \mathbb{N}[1, n)$. Then $\tilde{p}=n-p \in \mathbb{N}[1, n)$. Take $\tau \in \widetilde{\mathbb{C}}(p, n)$. Then $\tilde{\tau} \in \widetilde{\mathbb{C}}(\tilde{\mathrm{p}}, \mathrm{n})$. We have the surjective projection

$$
\pi \tilde{\tau}: \mathbb{D}(\mathbf{b}) \rightarrow \mathbb{D}\left(\pi_{\tau}(\mathbf{h})\right)
$$

For $z \in \mathbb{D}(\pi \sim(b))$ we have an injective holomorphic map

such that

$$
\begin{aligned}
& \tau_{z}: \mathbb{D}\left(\pi_{\tau}(\mathfrak{b})\right) \rightarrow \mathbb{D}(\mathfrak{b}) \\
& \tau_{z}: \mathbb{D}\left(\pi_{\tau}(\mathfrak{b})\right) \rightarrow \pi \frac{-1}{\tau}(z)
\end{aligned}
$$


is biholomorphic. Define

$$
\begin{aligned}
& R_{f}(\tau)=\left\{z \in \mathbb{D}\left(\pi_{\tilde{\tau}}(b)\right) \mid \tau_{z}^{-1}\left(I_{f}\right)=\mathbb{D}\left(\pi_{\tau}(b)\right)\right\} \\
& S_{f}(\tau)=\left\{z \in \mathbb{D}\left(\pi_{\tilde{\tau}}(b)\right) \mid \operatorname{dim} \tau_{z}^{-1}\left(I_{f}\right) \geq p-1\right\} .
\end{aligned}
$$

Then $R_{f}(\tau) \subseteq S_{f}(\tau)$. If $z \in \mathbb{D}\left(\pi \tau^{(b)}\right)-R_{f}(\tau)$, the meromorphic map $f$ restricts to a meromorphic map

$$
\mathbf{f}[\tau, \boldsymbol{z}]=\mathbf{f} \circ \tau_{z}: \mathbb{D}\left(\pi_{\tau}(\mathbf{b})\right) \rightarrow \mathbb{P}(\mathrm{V})
$$

If $z \in \mathbb{D}(\pi \tilde{\tau}(\mathbf{h}))-\mathrm{S}_{\mathbf{f}}(\tau)$, the reduced representation $\mathbb{w}: \mathbb{D}(\boldsymbol{b}) \rightarrow \mathrm{v}$ restricts to a reduced representation $\mathfrak{u} \circ \tau_{z}: \mathbb{D}\left(\pi_{\tau}(\mathrm{b})\right) \rightarrow \mathrm{V}$ of $f[\tau, z]$. By $(5.24)$ we have

$$
S_{f}(\tau)=\left\{z \in \mathbb{D}\left(\pi \tilde{\tau}^{(b)}\right) \mid \operatorname{dim} I_{f} \cap \pi_{\tilde{\tau}}^{-1}(z) \geq p-1\right\} .
$$

LEMMA 5.3. $\mathrm{S}_{\mathbf{f}}(\tau)$ is almost thin in $\mathbf{D}\left(\pi \tilde{\tau}^{(b))}\right.$.

PROOF. By [1] Theorem 1.14E $=\left\{\boldsymbol{x} \in \mathrm{I}_{\mathbf{f}}\left|\operatorname{rank}_{\boldsymbol{x}} \pi_{\tilde{\tau}}\right| \mathrm{I}_{\mathbf{f}} \leq \tilde{\mathrm{p}}-1\right\}$ is analytic in $\mathrm{I}_{\mathrm{f}}$ and by $[11]$ Lemma $1.30 \mathrm{E}^{\prime}=\pi_{\tilde{\tau}}(\mathrm{E})$ is almost thin. Take $z \in \mathrm{S}_{\mathrm{f}}(\tau)$. Then $x \in I_{f} \cap \pi_{\tilde{\tau}}^{-1}(z)$ exists such that $\operatorname{dim}_{\boldsymbol{x}} I_{f} \cap \pi_{\tilde{\tau}}^{-1}(z) \geq p-1$. An open, connected neighborhood $U$ of $x$ in $\mathbb{D}(b)$ exists such that $I_{f} \cap U=X_{1} \cup \ldots \cup x_{r}$ where each $X_{j}$ is a branch of $I_{f} \cap U$ with $x \in x_{j}$ for $j=1, \ldots, r$ and where $x_{j}$ is locally irreducible at $x$. A branch $N$ of $I_{f} \cap \pi_{\tilde{\tau}}^{-1}(z) \cap U$ exists with $x \in N$ and $\operatorname{dim}_{\boldsymbol{x}} N \geq p-1$. Then $\mathrm{N} \subseteq \mathrm{x}_{\mathrm{j}}$ for some $\mathrm{j}$. By $[1]$ Lemma 1.7 we obtain

$$
\begin{aligned}
\operatorname{rank}_{x} \pi_{\tilde{\tau}} \mid I_{f} & \leq \operatorname{rank}_{x} \pi \tilde{\tau} \mid x_{j}=\operatorname{dim} x_{j}-\operatorname{dim}_{x} x_{j} \cap \pi_{\tilde{\tau}}^{-1}(z) \\
& \leq \operatorname{dim} I_{f}-\operatorname{dim} N \leq n-2-(p-1)=\tilde{p}-1 .
\end{aligned}
$$

Therefore $x \in E$ and $z=\pi \sim(x) \in E^{\prime}$. Hence $S_{f}(\tau) \subseteq E^{\prime}$ is almost thin; q.e.d.

THEOREM 5.4. Take $\mathfrak{r} \in \stackrel{+}{\mathbb{R}}^{\mathbf{n}}(\mathfrak{h})$ and $\mathfrak{q} \in \stackrel{+}{\mathbf{n}}^{\mathbf{n}}(\mathfrak{h})$. Assume that $\mathfrak{p}=\pi \tilde{\tau}(\mathfrak{r})=\pi \tilde{\tau}(\mathfrak{q})$. Then

$$
\mathrm{T}_{\mathbf{f}}(\mathfrak{r}, \mathfrak{q})=\int_{z \in \mathbb{D}\langle\mathfrak{p}\rangle} \mathrm{T}_{\mathrm{f}[\tau, \boldsymbol{z}]}\left(\pi_{\tau}(\mathfrak{r}), \pi_{\tau}(\mathfrak{q})\right) \Omega_{\mathrm{p}}(\boldsymbol{z}) .
$$


PROOF. Let $\mathbf{u}: \mathbb{D}(\mathbf{b}) \rightarrow \mathrm{V}$ be a reduced representation of $\mathbf{f}$. Take

$z \in \mathbb{D}\langle\mathfrak{p}\rangle-\mathrm{S}_{\mathrm{f}}(\tau)$. Then $f[\tau, \boldsymbol{z}]$ exists and $\boldsymbol{u} \circ \tau_{\boldsymbol{z}}$ is a reduced representation of $f$

Therefore

$$
\mathrm{T}_{\mathrm{f}[\tau, \boldsymbol{z}]}\left(\pi_{\tau}(\mathbf{r}), \pi_{\tau}(\mathbf{q})\right)=\int_{\mathbf{D}\left\langle\pi_{\tau}(\boldsymbol{r})\right\rangle} \log \left\|\mathbf{u} \circ \tau_{\boldsymbol{z}}\right\| \Omega_{\mathbf{p}}-\int_{\mathbb{D}\left\langle\pi_{\tau}(\mathbf{q})\right\rangle} \log \left\|\mathbf{u} \circ \tau_{\boldsymbol{z}}\right\| \Omega_{\mathbf{p}} .
$$

Now (2.22) implies

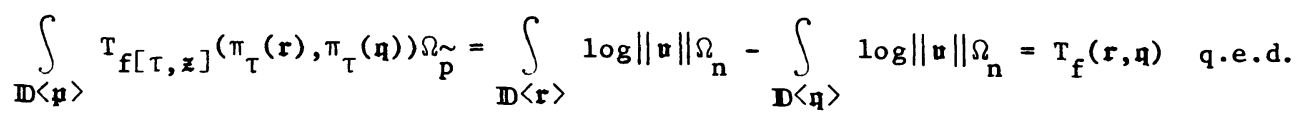

$$
\begin{aligned}
& \text { If } \pi_{\tau}(\boldsymbol{q})=q \pi_{\tau}(\boldsymbol{r}) \text { with } 0<q<1 \text {, then (5.14) implies } \\
& T_{f[\tau, z]}\left(\pi_{\tau}(r), q \pi_{\tau}(r)\right)=\int_{q}^{1} A_{f[\tau, z]}\left(t \pi_{\tau}(r)\right) \frac{d t}{t} .
\end{aligned}
$$

Define

$$
A_{f, \tau}\left(\mathfrak{p}, t \pi_{\tau}(\mathfrak{r})\right)=\int_{\mathfrak{z} \in \mathbf{D}\langle\mathfrak{p}\rangle} A_{f[\tau, \mathfrak{z}]}\left(t \pi_{\tau}(\mathfrak{r})\right) \frac{d t}{t}
$$

Then we have

$$
T_{f}(r, q)=\int_{q}^{1} A_{f, \tau}\left(p, t \pi_{\tau}(r)\right) \frac{d t}{t}
$$

provided $\pi_{\tau}(\boldsymbol{r})=\mathfrak{p}=\pi \tilde{\tau}(\mathfrak{q})$ and $\pi_{\tau}(\mathfrak{q})=q \pi_{\tau}(\mathfrak{r})$ with $0<q<1$.

Take $\mathrm{p}=1$ and identify $\tau \in \mathbb{C}(1, \mathrm{n})$ with $\tau(1) \in \mathbb{N}[1, \mathrm{n}]$. Then

$$
\int_{1}^{q} A_{f, \tau}\left(\mathfrak{p}, t \pi_{\tau}(r)\right) \frac{d t}{t}=\int_{q_{\tau}}^{r_{\tau}} A_{f, \tau}(p, t) \frac{d t}{t}
$$

If $r=\left(r_{1}, \ldots, r_{n}\right)$ and $q=\left(r_{1}, \ldots, r_{\tau-1}, q_{\tau}, r_{\tau+1}, \ldots, r_{n}\right)$, then

$$
T_{f}(r, q)=\int_{q_{\tau}}^{r_{\tau}} A_{f, \tau}(p, t) \frac{d t}{t}
$$

where $\mathfrak{p}=\left(\mathrm{r}_{1}, \ldots, \mathrm{r}_{\tau-1}, \mathrm{r}_{\tau+1}, \ldots, \mathrm{r}_{\mathrm{n}}\right)$ and

$$
A_{f \tau}(\mathfrak{p}, t)=\int_{z \in \mathbb{D}\langle\mathfrak{p}\rangle} A_{f[\tau, z]}(t) \frac{d t}{t}
$$




$$
A_{f[\tau, z]}(t)=\int_{\mathbb{D}[t]} f[\tau, z] *(\omega) .
$$

Take $0<\mathfrak{q}<\mathfrak{r}<\mathfrak{b}$ and define $\mathfrak{r}_{\tau}$ and $\mathfrak{p}_{\tau}$ as in (4.34) and (4.35). We obtain

$$
T_{f}(r, \mathfrak{q})=\sum_{\tau=1}^{n} \int_{q_{\tau}}^{r_{\tau}} A_{f, \tau}\left(p_{\tau}, t\right) \frac{d t}{t} .
$$

Take a $\in \mathbb{P}\left(V^{*}\right)$ and assume that $f$ is not linearly degenerate for a. Take $\mathrm{p} \in \mathbb{N}[1, \mathrm{n})$. Then $\tilde{\mathrm{p}}=\mathrm{n}-\mathrm{p} \in \mathbb{N}[1, \mathrm{n})$. Take $\tau \in \mathbb{\mathbb { e }}(\mathrm{p}, \mathrm{n})$. Then $\tilde{\tau} \in \mathbb{\mathbb { e }}(\tilde{\mathrm{p}}, \mathrm{n})$. Take $\mathfrak{b} \in \stackrel{+}{\mathbb{R}}^{\mathrm{n}}$ and $\mathfrak{r} \in \stackrel{+}{\mathbb{R}}^{\mathrm{n}}(\mathfrak{h})$. Take $z \in \mathbb{D}(\pi \tilde{\tau}(\mathfrak{b}))-\mathrm{S}_{\mathrm{f}}(\tau)$ such that $\mu_{\mathrm{f}}^{\mathrm{a}}$ is restrictable to $z$ by $\tau$. Then

$$
m_{f[\tau, z]}(\mathfrak{p}, a)=\int_{\mathbb{D}\langle\mathfrak{p}>} \log \frac{1}{\left\|f \circ \tau_{z}, a\right\|} \Omega_{p}
$$

is defined for all $\mathfrak{p} \in \stackrel{+}{\mathbb{R}}^{\mathrm{p}}\left(\pi_{\tau}(\mathfrak{h})\right)$. Then (2.22) implies

$$
\mathrm{m}_{\mathrm{f}}(\mathbf{r}, \mathrm{a})=\int_{z \in \mathbb{D}\langle\pi \sim \tilde{\tau}(r)\rangle} \mathrm{m}_{\mathrm{f}[\tau, z]}\left(\pi_{\tau}(r), a\right) \underset{\mathrm{p}}{\Omega \sim}(z) .
$$

\section{THE LEMMA OF THE LOGARITHMIC DERIVATIVE.}

Take $0<\mathrm{r}<\mathrm{R}<\mathrm{b} \leq+\infty$. Let $\mathrm{f}$ 丰 0 be a meromorphic function on $\mathbb{D}(\mathrm{b})$. By [9] Lemma 2.1 (see also Hayman [12], Lemma 2.3) we have

$$
\begin{aligned}
\int_{\mathbb{D}\langle\mathbf{r}\rangle} \log ^{+}\left|f^{\prime} / f\right| \Omega_{1} \leq & \log ^{+} m_{f}(R, 0)+\log ^{+} m_{f}(R, \infty)+3 \log ^{+} n_{f}(R, 0) \\
& +3 \log ^{+} n_{f}(R, \infty)+\log ^{+} \frac{R}{(R-r)^{2}}+\log ^{+} \frac{1}{r}+\log ^{+} \frac{r}{R-r}+8 \log 2 .
\end{aligned}
$$

We keep the notation of the last three sections. Take $\mathfrak{b} \in \stackrel{+}{\mathbb{R}}^{\mathrm{n}}$ and $\mathfrak{r}$ and $\mathbb{R}$ in $\stackrel{+}{\mathbb{R}}^{\mathrm{n}}(\mathrm{b}) . \quad$ Take $\tau \in \mathbb{N}[1, \mathrm{n}]$ and assume that

$$
0<\mathrm{r}=\pi_{\tau}(\mathfrak{r})<\pi_{\tau}(\mathbb{R})=\mathrm{R}<\mathrm{b} \quad \quad \pi \tilde{\tau}(\mathfrak{r})=\mathfrak{p}=\pi \tilde{\tau}(\mathbb{R}) .
$$


Let $\mathrm{f} \neq \mathrm{f} 0$ be a meromorphic function on $\mathbb{D}(\mathbf{b})$. Abbreviate

$$
\nu=\mu_{f}^{0} \quad \because=\mu_{f}^{\infty} \quad f_{\tau}^{\prime}=\frac{\partial f}{\partial z_{\tau}} .
$$

THEOREM 6.1. Under these assumptions we have

$$
\begin{aligned}
\int_{\mathbb{D}\langle\mathfrak{r}\rangle} \log ^{+}\left|f_{\tau}^{\prime} / f\right| \Omega_{n} \leq & \log ^{+} m_{f}(R, 0)+\log ^{+} m_{f}(R, \infty)+3 \log ^{+} n_{\nu, \tau}(\mathfrak{p}, R) \\
& +3 \log ^{+} n_{\mu, \tau}(\mathfrak{p}, R)+\log ^{+} \frac{R}{(R-r)^{2}}+\log ^{+} \frac{1}{r}+\log ^{+} \frac{r}{R-r}+16 \log 2 .
\end{aligned}
$$

PROOF. For $z \in \mathbb{D}\langle\mathfrak{p}\rangle-R_{f}(\tau)$ we have $f[\tau, z]=f \circ \tau_{z}$. The chain rule implies $f[\tau, z]^{\prime}=f_{\tau}^{\prime} \circ \tau_{z^{*}}$ Now $(2.22),(4.26)$ and $(5.38)$ imply

$$
\begin{aligned}
& \int_{\mathbb{D}<\mathbf{r}>} \log ^{+}\left|\mathbf{f}_{\tau}^{\prime} / \mathbf{f}\right| \Omega_{\mathbf{n}} \\
& =\int_{\mathbb{D}\langle\mathfrak{p}\rangle}\left(\int_{\mathbb{D}\langle\mathrm{r}\rangle} \log ^{+}|\mathrm{f}[\tau, z] \cdot / \mathrm{f}[\tau, z]| \Omega_{1}\right) \Omega_{\mathrm{n}-1}(z) \\
& \leq \int_{\mathbb{D}\langle\mathfrak{p}\rangle} \log ^{+} \mathrm{m}_{\mathrm{f}[\tau, \boldsymbol{z}]}(\mathrm{R}, 0) \Omega_{\mathrm{n}-1}+\int_{\mathbb{D}\langle\mathfrak{p}\rangle} \log ^{+} \mathrm{m}_{\mathrm{f}[\tau, \boldsymbol{z}]}(\mathrm{R}, \infty) \Omega_{\mathrm{n}-1}+3 \int_{\mathbb{D}\langle\mathfrak{p}\rangle} \log ^{+} \mathrm{n}_{\nu[\tau, \boldsymbol{z}]}(\mathrm{R}) \Omega_{\mathrm{n}-1} \\
& +3 \int_{\mathbb{D}\langle\mathfrak{p}\rangle} \log ^{+} n_{\mu[\tau, z]}(R) \Omega_{n-1}+\log ^{+} \frac{R}{(R-r)^{2}}+\log ^{+} \frac{1}{r}+\log ^{+} \frac{r}{R-r}+8 \log 2 \\
& \leq \log ^{+} \int_{\mathbb{D}\langle\mathfrak{p}\rangle} m_{\mathrm{f}[\tau, z]}(\mathrm{R}, 0) \Omega_{\mathrm{n}-1}+\log ^{+} \int_{\mathbb{D}\langle\boldsymbol{p}\rangle} \mathrm{m}_{\mathrm{f}[\tau, \boldsymbol{z}]}(\mathrm{R}, \infty) \Omega_{\mathrm{n}-1}+3 \log ^{+} \int_{\mathbb{D}\langle\boldsymbol{p}\rangle} \mathrm{n}_{\nu[\tau, \boldsymbol{z}]}(\mathrm{R}) \Omega_{\mathrm{n}-1}
\end{aligned}
$$

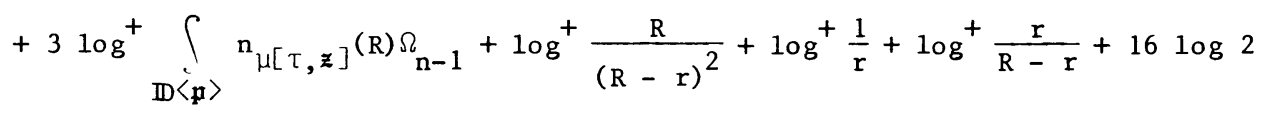

$$
\begin{aligned}
& =\log ^{+} \mathrm{m}_{\mathrm{f}}(\mathbb{R}, 0)+\log ^{+} \mathrm{m}_{\mathrm{f}}(\mathbb{R}, \infty)+3 \log ^{+} \mathrm{n}_{\nu, \tau}(\mathfrak{p}, \mathrm{R})+3 \log ^{+} \mathrm{n}_{\mu, \tau}(\mathfrak{p}, \mathrm{R}) \\
& +\log ^{+} \frac{R}{(R-r)^{2}}+\log ^{+} \frac{1}{r}+\log ^{+} \frac{r}{R-r}+16 \log 2
\end{aligned}
$$

THEOREM 6.2. If $0<\mathfrak{q} \leq \mathfrak{r}$, we obtain under the same assumptions

$$
\begin{aligned}
\int_{\mathbb{D}\langle\mathfrak{r}\rangle} \log ^{+}\left|\mathrm{f}_{\tau}^{\prime} / \mathrm{f}\right| \Omega_{\mathrm{n}} \leq & 8 \log ^{+} \mathrm{T}_{\mathrm{f}}(\mathbb{R}, \mathfrak{q})+4 \log ^{+} \mathrm{m}_{\mathrm{f}}(\mathfrak{q}, 0)+4 \log ^{+} \mathrm{m}_{\mathrm{f}}(\mathfrak{q}, \infty) \\
& +9 \log \frac{2 R}{R-r}+2 \log ^{+} \frac{1}{r}+24 \log 2 .
\end{aligned}
$$


PROOF. Define $E=(R+r) / 2 R<1$. Then $r<\theta R<R$ and $\theta R-r=(R-r) / 2$. Define

$$
\hat{\mathbf{r}}=\left(r_{1}, \ldots, r_{\tau-1}, \Theta_{R, r_{\tau+1}}, \ldots, r_{n}\right)
$$

Then $\mathbf{r} \leq \hat{\mathbf{r}} \leq \mathbb{R}$ and $\pi_{\tau}(\mathfrak{r})=\mathbf{r}<\pi_{\tau}(\hat{\boldsymbol{r}})=\theta \mathbb{R}<\pi_{\tau}(\mathbb{R})=\mathbb{R}$ and $\pi_{\tau}(\mathfrak{r})=\pi_{\tau}(\tilde{\mathfrak{r}})=\pi_{\tau}(\mathbb{R})=\mathfrak{p}$. Since $N_{V}(\hat{r}, q) \geq 0$, the First Main Theorem implies

$$
\begin{aligned}
& m_{f}(\hat{r}, 0) \leq T_{f}(\hat{r}, \mathfrak{q})+m_{f}(\mathfrak{q}, 0) \leq T_{f}(R, q)+m_{f}(\mathfrak{q}, 0) \\
& \log ^{+} m_{f}(\hat{r}, 0) \leq \log ^{+} T_{f}(R, q)+\log ^{+} m_{f}(\mathfrak{q}, 0)+\log 2 \\
& \log ^{+} m_{f}(\hat{r}, \infty) \leq \log ^{+} T_{f}(R, q)+\log ^{+} m_{f}(\mathfrak{q}, \infty)+\log 2 .
\end{aligned}
$$

By (4.31) we have

$$
\begin{aligned}
& N_{v}(R, q)=N_{v}(R, r)+N_{v}(r, q) \geq N_{v}(R, r)=\int_{r}^{R} n_{\nu, \tau}(p, t) \frac{d t}{t} \geq \int_{\theta R}^{R} n_{\nu, \tau}(p, t) \frac{d t}{t} \\
& \geq n_{\nu, \tau}(\mathfrak{p}, \Theta R) \frac{R-\theta R}{R}=n_{\nu, \tau}(\mathfrak{p}, \Theta R) \frac{R-r}{2 R} \\
& \log ^{+} n_{\nu, \tau}(\mathfrak{p}, \Theta R) \leq \log ^{+} N_{\nu}(R, \mathfrak{q})+\log ^{+} \frac{2 R}{R-r} \leq \log ^{+}\left(T_{f}(R, \mathfrak{q})+m_{f}(\mathfrak{q}, 0)\right)+\log \frac{2 R}{R-r}
\end{aligned}
$$

or

$$
\begin{aligned}
& \log ^{+} n_{\nu, \tau}(\mathfrak{p}, \Theta R) \leq \log ^{+} T_{f}(\mathbb{R}, \mathfrak{q})+\log ^{+} m_{f}(\mathfrak{n}, 0)+\log \frac{2 R}{R-r}+\log 2 \\
& \log ^{+} n_{\mu, \tau}(\mathfrak{p}, \Theta R) \leq \log ^{+} T_{f}(\mathbb{R}, \mathfrak{q})+\log ^{+} m_{f}(\mathfrak{q}, \infty)+\log \frac{2 R}{R-r}+\log 2 .
\end{aligned}
$$
Also we have

$$
\begin{aligned}
\log ^{+} \frac{\Theta R}{(\Theta R-r)^{2}} & \leq \log ^{+} \frac{\theta R}{\Theta R-r}+\log ^{+} \frac{R}{\Theta R-r}+\log ^{+} \frac{1}{R} \\
& =\log ^{+} \frac{R+r}{R-r}+\log ^{+} \frac{2 R}{R-r}+\log ^{+} \frac{1}{R} \leq 2 \log \frac{2 R}{R-r}+\log ^{+} \frac{1}{r} \\
\log ^{+} \frac{r}{R-r} & \leq \log \frac{2 R}{R-r} .
\end{aligned}
$$

Now, Theorem 6.1 for $\mathfrak{r}$ and $\hat{\mathfrak{r}}$ and $(6.6)-(6.11)$ imply (6.5), q.e.d. 


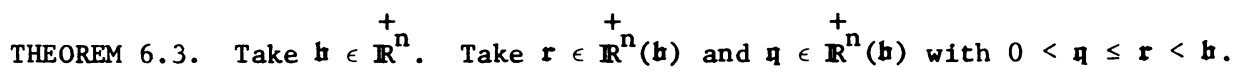
Take $1<\theta \in \mathbb{R}$ and $0<q \in \mathbb{R}$ such that $0<\mathbf{q} \cdot \mathbf{h} \leq \mathbf{q} \leq \boldsymbol{r}<\theta \boldsymbol{r}<\mathbf{b}$. Let f $\mathbf{f} 0$ be a meromorphic function on $\mathbb{D}(\mathfrak{b})$. Take $\tau \in \mathbb{N}[1, n]$. Define $\mathbf{f}_{\tau}^{\prime}=\frac{\partial f}{\partial z_{\tau}}$. Then

$$
\begin{aligned}
\int_{\mathbb{D}\langle\mathbf{r}\rangle} \log ^{+}\left|f_{\tau}^{\prime} / f\right| \Omega_{n} \leq & 8 \log ^{+} T_{f}(\Theta r, q)+4 \log ^{+} m_{f}(\mathfrak{q}, 0)+4 \log _{m_{f}}^{+}(\mathfrak{q}, \infty) \\
& +9 \log \frac{2 \theta}{\theta-1}+2 \log ^{+} \frac{1}{q^{b} b_{\tau}}+24 \log 2 .
\end{aligned}
$$

PROOF. Theorem 6.2 can be applied with $\mathbf{r}=\mathbf{r}_{\tau}$ and $R=\theta \mathbf{r}_{\tau}$ and

$$
\mathbb{R}=\left(\mathrm{r}_{1}, \ldots, \mathrm{r}_{\tau-1}, \mathrm{R}, \mathrm{r}_{\tau+1}, \ldots, \mathrm{r}_{\mathrm{n}}\right)
$$

Then $\mathbb{R} \leq \theta \mathbf{r}$ and $T_{f}(R, \mathfrak{q}) \leq T_{f}(\Theta r, \mathfrak{q})$. Also $2 R /(R-r)=2 \theta /(\Theta-1)$ and $1 / \mathbf{r} \leq 1 / q b{ }_{\tau}$. Consequently (6.5) implies (6.12); q.e.d.

Theorem 6.3 is a preliminary version of the Lemma of the logarithmic derivative. For $r$ to approach $h$, we have to eliminate $\theta$. If we restrict ourself to the approach $\mathrm{rh} \rightarrow \mathrm{h}$ with $1>\mathrm{r} \rightarrow 1$ this could be accomplished along classical lines and would result in the usual "exceptional intervals". Rubel and Henson want to consider the approach $\mathbf{b}>\mathbf{r} \rightarrow \mathbf{b}$. In order to satisfy both cases, we introduce a more general method resulting in "exceptional sets" of dimensions between 1 and $n$.

$$
\begin{array}{r}
\text { Take } \mathbf{b} \in \stackrel{+}{\mathrm{R}}^{\mathrm{n}} \text { and } \mathrm{p} \in \mathbb{Z}[0, \mathrm{n}) . \text { Define } \delta: \mathbb{R}_{+}^{\mathrm{n}}(\mathbf{b}) \rightarrow \mathbb{R}(0,1] \text { by } \\
\delta(\boldsymbol{x})=\operatorname{Min}\left\{1-\left(\mathrm{x}_{\mathrm{j}} / \mathrm{b}_{\mathrm{j}}\right) \mid \mathrm{j}=1, \ldots, \mathrm{n}\right\}
\end{array}
$$

for $x \in \underset{+}{\mathbb{R}^{n}(b)}$. Let B be a real, pure $p$-dimensional, oriented submanifold of class $C^{k}$ in $\mathbb{R}^{\mathrm{n}}$ with $\mathrm{k} \geq 1$. Denote by $\mu_{B}$ the differential geometric measure on $B$. It equals the p-dimensional Hausdorff measure. For $\boldsymbol{x} \in \mathrm{B}$, let $\mathrm{T}_{\boldsymbol{x}}$ be the real tangent space and $N_{\boldsymbol{x}}$ the normal space of $\mathrm{B}$ at $\boldsymbol{x}$. Then we have the orthogonal decomposition $\mathbf{R}^{\mathrm{n}}=\mathrm{T}_{\boldsymbol{x}} \oplus \mathrm{N}_{\boldsymbol{x}}$. Let $\rho_{\boldsymbol{x}}: \mathbb{R}^{\mathrm{n}} \rightarrow \mathrm{N}_{\mathbf{x}}$ be the projection. The manifold $\mathrm{B}$ is said to be an approach base if the following conditions are satisfied. 
(a) The manifold $B$ has finite volume $\mu_{B}(B)<\infty$.

(b) If $\boldsymbol{x} \in \mathrm{B}$, then $0 \leq \boldsymbol{x}<\mathfrak{h}$, that is, $\mathrm{B} \subseteq \mathbb{R}^{\mathrm{n}}(\mathbf{b})$.

(c) If $\boldsymbol{x} \in \overline{\mathrm{B}}$ and $t \in \mathbb{R}$ and $\boldsymbol{x}+\boldsymbol{t}(\boldsymbol{b}-\boldsymbol{x}) \in \overline{\mathrm{B}}$, then $\boldsymbol{t}=0$.

(d) If $p>0$, there is a number $\gamma>0$ such that for each $\boldsymbol{x} \in \mathrm{B}$ we have

$$
\left\|\rho_{\boldsymbol{x}}(\boldsymbol{b}-\boldsymbol{x})\right\| \geq \gamma \text {. }
$$

(e) If $p>0$, then

$$
\int_{B} \log (1 / \delta) d \mu_{B}<\infty .
$$

The closure $\bar{B}$ is compact and contained in $\mathbb{R}_{+}^{n}[\mathbf{b}]$. If $\mathbf{b} \in \bar{B}$, then $\mathbf{b}=\mathbf{b}+(\mathbf{b}-\mathbf{b}) \epsilon \bar{B}$ which contradicts (c). Hence $b \notin \bar{B}$.

Define the associated approach cone by

$$
M=\{\boldsymbol{x}+\boldsymbol{t}(\boldsymbol{b}-\boldsymbol{x}) \mid \boldsymbol{x} \in B \text { and } \boldsymbol{t} \in \mathbb{R}(0,1)\}
$$

If $\boldsymbol{x} \in \mathrm{B}$, then $\boldsymbol{x}<\mathbf{b}$ and $\boldsymbol{x}<\boldsymbol{x}+\mathrm{t}(\boldsymbol{b}-\boldsymbol{x})<\mathfrak{b}$ for $0<t<1$. Hence $M \subseteq \mathbb{R}^{\mathbf{n}}(\boldsymbol{b})$. EXAMPLE 1. Take

$$
\begin{aligned}
& B=\left\{x \in \mathbb{R}^{n}(b) \mid \sum_{j=1}^{n} x_{j} / b_{j}=1\right\} \\
& M=\left\{x \in \mathbb{R}_{+}^{n}(b) \mid \sum_{j=1}^{n} x_{j} / b_{j}>1\right\} .
\end{aligned}
$$

Simple calculations show that the conditions (a)-(e) are satisfied. Hence $B$ is an approach base and $M$ the associated approach cone. Observe that $M \cup\{\boldsymbol{b}\}$ is an open neighborhood of $\mathfrak{b}$ in $\mathbb{R}^{\mathbf{n}}(\mathfrak{h}) \cup\{\mathfrak{h}\}$.

EXAMPLE 2. Take $B=\{0\}$. Then $M=\{t b \mid 0<t<1\}$.

While the geometric meaning of the conditions $(a)-(d)$ is obvious, the condition (e) is more difficult to analyze. For this purpose we offer the following considerations:

LEMMA 6.4. Let $B$ be a real, pure p-dimensional, oriented submanifold of class 
$\mathrm{C}^{\mathrm{k}}$ in $\mathbb{R}^{\mathrm{n}}$ with $\mathrm{k} \geq 1$, with $\mathrm{p} \geq 1$ and with $\mu_{B}(\mathrm{~B})<\infty$. For each $\lambda \in \mathbb{R}(0,1]$ define

$$
\begin{aligned}
& B(\lambda)=B- \mathbb{R}^{n}((1-\lambda) \mathbf{h}) . \\
&+
\end{aligned}
$$

Assume there are constants $c>0$ and $q>0$ such that $\mu_{B}(B(\lambda)) \leq c \lambda^{q}$ for all

$\lambda \in \mathbb{R}(0,1]$. Then $(6.15)$ is satisfied.

PROOF. Observe that $B(1)=B$ and $B(\lambda)=\{\boldsymbol{x} \in \mathrm{B} \mid \delta(\boldsymbol{x}) \leq \lambda\}$. For $\mathrm{m} \in \mathbb{N}$ we have

$$
\begin{aligned}
A(m) & =B(1 / m)-B(1 /(m+1))=\{x \in B \mid 1 /(m+1)<\delta(x) \leq 1 / m\} \\
d_{m} & =\mu_{B}(B(1 / m)) \leq \mathrm{cm}^{-q} \\
a_{m} & =\mu_{B}(A(m))=d_{m}-d_{m+1} .
\end{aligned}
$$

This yields the estimate

$$
\begin{aligned}
\int_{B} \log (1 / \delta) d \mu_{B} & \leq \sum_{m=1}^{\infty} \int_{A_{m}} \log (m+1) d \mu_{B}=\sum_{m=1}^{\infty} a_{m} \log (m+1) \\
& =\lim _{r \rightarrow \infty}\left(\sum_{m=1}^{r-1} d_{m} \log (m+1)-\sum_{m=1}^{r-1} d_{m+1} \log (m+1)\right) \\
& =\lim _{r \rightarrow \infty}\left(\sum_{m=1}^{r} d_{m} \log (1+1 / m)-d_{r} \log (r+1)\right) \\
& \leq c \sum_{m=1}^{\infty} m^{-q} \log (1+1 / m) \leq c \sum_{m=1}^{\infty} m^{-1-q<\infty}
\end{aligned}
$$

A map $\mathfrak{y}: \mathbb{R} \times \mathbb{R}^{n} \rightarrow \mathbb{R}$ is defined by $\mathbf{y}(t, \boldsymbol{x})=\boldsymbol{x}+t(\mathfrak{b}-\boldsymbol{x})$ for $t \in \mathbb{R}$ and $\boldsymbol{x} \in \mathbb{R}^{\mathrm{n}}$. Obviously $\mathfrak{y}(0, \boldsymbol{x})=\boldsymbol{x}$ and $\boldsymbol{y}(1, \boldsymbol{x})=\mathbf{b}$. If $0<\mathrm{s}<\mathrm{t}<1$ then $\boldsymbol{x}<\boldsymbol{y}(t, \boldsymbol{x})<\mathbf{b}$. By definition $M=y(\mathbb{R}(0,1) \times B)$.

LEMMA 6.5. The map $y:(\mathbb{R}-\{1\}) \times \bar{B} \rightarrow \mathbb{R}^{n}$ is injective.

PROOF. Assume that $\boldsymbol{x}$ and $\tilde{\boldsymbol{x}}$ exist in $\bar{B}$ and $1 \neq t \in \mathbb{R}$ and $1 \neq \tilde{t} \in \mathbb{R}$ such that $\boldsymbol{y}(t, \boldsymbol{x})=\boldsymbol{y}(\tilde{t}, \tilde{x})$. Then $t(\mathbf{h}-\boldsymbol{x})=\tilde{\boldsymbol{x}}-\boldsymbol{x}+\tilde{t}(\mathbf{b}-\tilde{\boldsymbol{x}})$. Hence

$$
x+\frac{t-\tilde{t}}{1-\tilde{t}}(b-x)=x+\frac{1}{1-\tilde{t}}(\tilde{x}-x+\tilde{t}(\mathfrak{b}-\tilde{x})-\tilde{t}(b-x))=\tilde{x} \epsilon \bar{B} .
$$

Hence $t=\tilde{t}$ and $\boldsymbol{x}=\tilde{\boldsymbol{x}} ; q \cdot e \cdot d$. 
LEMMA 6.6. The map $y: \mathbb{R}(0,1) \times B \rightarrow M$ is a homeomorphism.

PROOF. By definition the map is surjective, hence by Lemma 6.5 the map y $: \mathbb{R}(0,1) \times B \rightarrow M$ is bijective. Trivially the map is continuous. It remains to be shown that $\mathfrak{y}$ is open. Let $U$ be an open subset of $\mathbb{R}(0,1) \times B$. Take $(t, x) \in U$. Assume that $y(t, x)$ is not an interior point of $y(U)$ in $M$. A sequence $\left\{\left(t_{V}, x_{V}\right)\right\}_{V \in \mathbb{N}}$ exists in $\mathbb{R}(0,1) \times B-U$ such that $\mathfrak{y}\left(t_{V}, x_{V}\right) \rightarrow \mathfrak{y}(t, x)$ for $v \rightarrow \infty$. By going to a subsequence we can assume that $t_{v} \rightarrow \tilde{t}$ and $x_{v} \rightarrow \tilde{x}$ for $\nu \rightarrow \infty$ with $(\tilde{t}, \tilde{x}) \in \mathbb{R}[0,1] \times \bar{B}-U$. Then $\mathfrak{y}(t, x)=\mathfrak{y}(\tilde{t}, \tilde{x})$. If $\tilde{t}=1$, then $\mathbf{b}=\mathbf{y}(1, \tilde{x})=\mathfrak{y}(t, x) \in M$ which is wrong. Therefore $\tilde{t} \neq 1$ and $(\tilde{t}, \tilde{x})=(t, x) \in U$ which is impossible. Therefore $y(U)$ is open in $M$; q.e.d.

Let $V \neq \emptyset$ be an open, connected subset of $\mathbb{R}^{\mathrm{P}}$. Let $U \neq \emptyset$ be an open subset of $B$ and let $\mathfrak{g}: \mathrm{V} \rightarrow \mathrm{U}$ be an orientation preserving diffeomorphism of class $\mathrm{C}^{1}$. Let $\mathfrak{g}_{\mathbf{j}}$ be the partial derivatives of $\mathfrak{g}$. Define $\mathfrak{w}: \mathbb{R} \times \mathrm{V} \rightarrow \mathbb{R}^{\mathrm{n}}$ by $\mathfrak{w}(t, \mathfrak{u})=\mathfrak{y}(t, \mathfrak{g}(\mathfrak{u}))$ for $t \in \mathbb{R}$ and $\mathbf{u} \in \mathrm{V}$. Then

$$
\begin{array}{rc}
\mathfrak{w}_{t}(t, \mathfrak{u})=\mathbf{w}-\mathfrak{g}(\mathfrak{u}) & \mathfrak{w}_{v_{j}}(t, \mathfrak{u})=(1-t) g_{v_{j}}(\mathfrak{u}) . \\
\mathfrak{w}_{t}(t, \mathfrak{u}) \wedge \mathfrak{w}_{v_{1}}(t, \mathfrak{u}) \wedge \ldots \wedge \mathfrak{w}_{v_{p}}(t, \mathfrak{u})=(1-t)^{p}(\mathfrak{w}-\mathfrak{g}(\mathfrak{u})) \wedge g_{v_{1}}(\mathfrak{u}) \wedge \ldots \wedge g_{v_{p}}(u) .
\end{array}
$$

The vectors $g_{v_{1}}(u), \ldots, g_{v_{p}}(u)$ span $T_{\mathfrak{g}(\mathfrak{u})}$. By $(d)$, we have $\mathbf{b}-\mathfrak{g}(\mathfrak{u}) \notin \mathrm{T}_{\mathfrak{g}(\mathfrak{u})}$. Therefore

$$
(\mathbf{b}-\mathfrak{g}(\mathfrak{u})) \wedge \mathfrak{g}_{\mathrm{v}_{1}}(\mathfrak{u}) \wedge \ldots \wedge \mathfrak{g}_{\mathrm{v}_{\mathrm{p}}}(\mathfrak{u}) \neq 0
$$

In fact, if $\boldsymbol{x}=\mathbf{g}(\mathbf{u})$, then

$$
\Delta(\boldsymbol{x})=\frac{\left\|(\mathfrak{w}-\mathfrak{g}(\mathfrak{u})) \wedge \mathfrak{g}_{\mathrm{v}_{1}}(\mathfrak{u}) \wedge \ldots \wedge \mathfrak{g}_{\mathrm{v}_{\mathrm{p}}}(\mathfrak{u})\right\|}{\left\|\mathfrak{g}_{\mathrm{v}_{1}}(\mathfrak{u}) \wedge \ldots \wedge \mathfrak{g}_{\mathrm{v}_{\mathrm{p}}}(\mathfrak{u})\right\|}
$$

depends only on $\boldsymbol{x}$ and not on the parameterization $\mathfrak{g}$. Hence $\Delta: B \rightarrow \mathbb{R}$ is globally defined. 


$$
0<\gamma \leq \Delta(\boldsymbol{x})=\left\|\rho_{\boldsymbol{x}}(\boldsymbol{b}-\boldsymbol{x})\right\| \leq\|\mathbf{b}\|
$$

PROOF. We have $\mathfrak{b}-\boldsymbol{x}=\rho_{\boldsymbol{x}}(\boldsymbol{b}-\boldsymbol{x})+\sum_{\mathbf{j}=1}^{p} a_{j} \mathbf{g}_{v_{j}}$ (u). Hence

$$
\left\|(\mathbf{b}-\boldsymbol{x}) \wedge \mathrm{g}_{\mathrm{v}_{1}}(u) \wedge \ldots \wedge \mathrm{g}_{\mathrm{v}_{\mathrm{p}}}(u)\right\|=\left\|\rho_{\mathbf{x}}(\mathbf{b}-\mathbf{x})\right\|\left\|g_{\mathrm{v}_{1}}(u) \wedge \ldots \wedge \mathrm{g}_{\mathrm{v}_{\mathrm{p}}}(u)\right\|
$$
or $\Delta(\boldsymbol{x})=\left\|\rho_{\mathbf{x}}(\boldsymbol{h}-\boldsymbol{x})\right\|$. By $(6.14)$ we have $\Delta(\boldsymbol{x}) \geq \gamma$. Also

$$
\Delta(\boldsymbol{x})^{2}=\left\|\rho_{x}(\mathbf{h}-\boldsymbol{x})\right\|^{2} \leq\|\boldsymbol{b}-\boldsymbol{x}\|^{2}=\sum_{j=1}^{n}\left(b_{j}-x_{j}\right)^{2} \leq \sum_{j=1}^{n} b_{j}^{2}=\|\mathbf{b}\|^{2}
$$

which implies $\Delta(\boldsymbol{x}) \leq\|\mathbf{b}\|$, q.e.d.

By (6.18) and (6.19) the map $y: \mathbb{R}(0,1) \times B \rightarrow \mathbb{R}^{n}$ is smooth. In conjunction with Lemma 6.6 we have proved:

THEOREM 6.8. $M$ is an embedded, oriented, differentiable manifold of pure dimension $p+1$ and of class $C^{1}$ such that $y: \mathbb{R}(0,1) \times B \rightarrow M$ is an orientation preserving diffeomorphism of class $c^{1}$.

Let $\mu_{M}$ and $\mu_{B}$ be the differential geometric measures on $M$ and $B$ respectively. In the situation $(6.17)-(6.20)$ we have

$$
\begin{aligned}
& \mathrm{d} \mu_{\mathrm{B}}=\left\|\mathrm{g}_{\mathrm{v}_{1}} \wedge \ldots \wedge \mathrm{g}_{\mathrm{v}_{\mathrm{p}}}\right\| \mathrm{dv_{1 }} \wedge \ldots \wedge \mathrm{dv}_{\mathrm{p}} \\
& \mathrm{d} \mu_{\mathrm{M}}=(1-\mathrm{t})^{\mathrm{p}}\left\|(\boldsymbol{b}-\mathrm{g}) \wedge \mathrm{g}_{\mathrm{v}_{1}} \wedge \ldots \wedge \mathrm{g}_{\mathrm{v}_{\mathrm{p}}}\right\| \mathrm{dt} \wedge \mathrm{dv_{1 }} \wedge \ldots \wedge \mathrm{dv_{p }} .
\end{aligned}
$$

Hence globally

$$
d \mu_{M}=(1-t)^{P} \Delta(x) d t \wedge d \mu_{B}
$$

Now, Lemma 6.7 implies

$$
0 \leq \frac{\gamma}{p+1} \mu_{B}(B) \leq \mu_{M}(M) \leq \frac{\|\boldsymbol{b}\|}{p+1} \mu_{B}(B)<\infty .
$$

Here we are able to give another example where the conditions (a)-(d) imply condition (e). 
PROPOSITION 6.9. Take $\mathfrak{h} \in \stackrel{+}{\mathbb{R}}^{n}$. Let $A$ be a pure $(n-1)$-dimensional, oriented submanifold of class $C^{k}$ in $\mathbb{R}^{n}$ with $k \geq 1$. Assume that $B=A \cap \mathbb{R}^{n}(b)$ is relative compact in $A$. Then $\mu_{B}(B)<\infty$. Assume that there exists a number $\gamma>0$ such that (6.14) holds. Then (6.15) is satisfied. If also condition (c) holds, then B is an approach base.

PROOF. Define $B_{j}=\left\{x \in B \mid \delta(x)=1-\left(x_{j} / b_{j}\right)\right\}$ for $j=1, \ldots, n$. Then $B=B_{1} \cup \ldots \cup B_{n} \cdot$ Each $\bar{B}_{j}$ is a compact subset of A. Take a $\epsilon \bar{B}_{j}$ with $a_{j}=b_{j}$. Then there exists an orientation preserving diffeomorphism $\mathfrak{g}: \mathrm{V} \rightarrow \mathrm{U}$ of $\mathrm{class}^{\mathrm{l}} \mathrm{C}^{\mathrm{a}}$ where $U$ is an open neighborhood of $a$ in $A$ and where $V$ is an open neighborhood of 0 in $\mathbb{R}^{\mathrm{n}-1}$ and where $\mathfrak{g}(0)=\mathfrak{a}$. Let $\nabla$ be the gradient. Abbreviate

$$
\begin{aligned}
G & =\left\|g_{v_{1}} \wedge \ldots \wedge g_{v_{n-1}}\right\|>0 \\
G_{i} & =(-1)^{i-1} \operatorname{det}\left(\nabla g_{1}, \ldots, \nabla g_{i-1}, \nabla g_{i+1}, \ldots, \nabla g_{n}\right) .
\end{aligned}
$$

Then $(6.20)$ reads

$$
(\Delta \circ \mathfrak{g}) \mathrm{G}=\left|\sum_{i=1}^{n}\left(b_{i}-g_{i}\right) G_{i}\right| .
$$

Assume that $\nabla g_{j}(0)=0$. Since $\Delta(\boldsymbol{x}) \geq \gamma>0$ for all $\boldsymbol{x} \in \mathrm{B}$, we have $\Delta(\mathfrak{a}) \geq \gamma>0$ by continuity. Also $\nabla g_{j}(0)=0$ implies $G_{i}(0)=0$ for all $i \neq j$. Therefore

$$
0<\Delta(a) G(0)=\left(b_{j}-g_{j}(0)\right) G_{j}(0)=\left(b_{j}-a_{j}\right) G_{j}(0)=0
$$

This contradiction shows that $\nabla_{g_{j}}(0) \neq 0$. Therefore $d\left(b_{j}-x_{j}\right) \neq 0$ at a. Consequently $\log \left(b_{j}-x_{j}\right)$ is locally integrable at $a \in \bar{B}_{j}$ if $a_{j}=b_{j} \cdot$ If $a_{j} \neq b_{j}$, then $a_{j}<b_{j}$ and $\log \left(b_{j}-x_{j}\right)$ is trivially locally integrable at a. Since $\bar{B}_{j}$ is compact, $\log \left(b_{j}-x_{j}\right)$ is integrable over $B_{j}$. Therefore

$$
\int_{B} \log (1 / \delta) d \mu_{B} \leq \sum_{j=1}^{n} \int_{B_{j}} \log \frac{b_{j}}{b_{j}-x_{j}} d \mu_{B}<\infty, \quad \text { q.e.d. }
$$

If $E$ is a measurable subset of $M$, then the set

$$
E_{\boldsymbol{x}}=\{t \in \mathbb{R}(0,1) \mid \boldsymbol{x}+\mathrm{t}(\boldsymbol{b}-\boldsymbol{x}) \in \mathrm{E}\}
$$


is measurable for almost all $\boldsymbol{x} \in \mathrm{B}$. Let $\mathrm{g}: \mathrm{E} \rightarrow \mathbb{R}$ be integrable over $\mathrm{E}$. Fubini's theo rem implies

$$
\int_{E} g d \mu_{M}=\int_{x \in B}\left(\int_{E(x)} g(y(t, x))(1-t)^{p} d t\right) \Delta(x) d \mu_{B}(x) .
$$

Define

$$
\Lambda_{M}(E)=\int_{r \in E} \frac{d \mu_{M}(\mathfrak{r})}{\|\mathbf{h}-\mathfrak{r}\|^{p+1}}=\int_{\boldsymbol{r} \in \mathrm{B}}\left(\int_{E(\boldsymbol{x})} \frac{\mathrm{d} t}{1-t}\right) \frac{\Delta(\boldsymbol{x}) \mathrm{d} \mu_{\mathrm{B}}(\boldsymbol{x})}{\|\mathbf{h}-\boldsymbol{x}\|^{\mathrm{p}+1}} .
$$

Then $0 \leq \Lambda_{M}(E) \leq \infty$.

LEMMA 6.10. Define $\gamma_{0}=\operatorname{Min}\{\|\mathbf{h}-\boldsymbol{x}\| \mid \boldsymbol{x} \in \bar{B}\}$. Then $\gamma_{0}>0$. Define

$$
\gamma_{1}=\left(1 / \gamma_{0}\right)^{\mathrm{p}} \quad \gamma_{2}=\gamma\|\mathbf{b}\|^{-\mathrm{p}-1}
$$

Let $E$ be a measurable set on $M$. Then

$$
\gamma_{2} \int_{\boldsymbol{x} \in \mathbf{B}}\left(\int_{E(\boldsymbol{x})} \frac{\mathrm{dt}}{1-\mathrm{t}}\right) \mathrm{d} \mu_{B}(\boldsymbol{x}) \leq \Lambda_{M}(E) \leq \gamma_{1} \int_{\boldsymbol{x} \in \mathrm{B}}\left(\int_{E(\boldsymbol{x})} \frac{\mathrm{dt}}{1-t}\right) \mathrm{d} \mu_{B}(\boldsymbol{x}) .
$$

PROOF. Recall that $0<\gamma \leq \Delta(\boldsymbol{x}) \leq\|\mathbf{h}-\boldsymbol{x}\| \leq\|\mathbf{b}\|$. Hence

$$
\gamma_{2}=\frac{\gamma}{\|\mathbf{b}\|^{p+1}} \leq \frac{\Delta(x)}{\|\mathbf{b}-\boldsymbol{x}\|^{p+1}} \leq \frac{1}{\|\mathbf{b}-\boldsymbol{x}\|^{p}} \leq\left(\frac{1}{\gamma_{0}}\right)^{p}=\gamma_{1}
$$

which with (6.25) implies (6.26); q.e.d.

For $0<\mathrm{r} \leq \mathrm{s} \leq 1$ define

$$
\begin{array}{ll}
M[r, s]=y(\mathbb{R}[r, s] \times B) & M(r, s)=y(\mathbb{R}(r, s) \times B) \\
M(r, s]=y(\mathbb{R}(r, s] \times B) & M[r, s)=y(\mathbb{R}[r, s) \times B) .
\end{array}
$$

Then $M=M(0,1)$ and $M \cup\{\mathfrak{h}\}=M(0,1]$. If $t_{j} \in \mathbb{R}(0,1)$ for $j \in \mathbb{N}$ and if $t_{j} \rightarrow 1$ for $j \rightarrow \infty$, then $\left\{M\left(t_{j}, 1\right]\right\}_{j \in \mathbb{N}}$ is a base of open neighborhoods of $\mathbf{b}$ in $M \cup\{\mathbf{b}\}$. If $0<\mathrm{r} \leq \mathrm{s}<1$, then (6.26) implies

$$
\begin{aligned}
& \gamma_{2} \mu_{B}(B) \log \frac{1-r}{1-s} \leq \Lambda_{M}(M[r, s]) \leq \gamma_{1} \mu_{B}(B) \log \frac{1-r}{1-s} \\
& \Lambda_{M}(M[r, 1))=\infty \\
& \Lambda_{M}(M(0, s])<\infty .
\end{aligned}
$$


LEMMA 6.11. Define

$$
Q=\{\boldsymbol{y}(t, \boldsymbol{x}) \mid \boldsymbol{x} \in \mathrm{B} \text { and } 0<t<1-\delta(\boldsymbol{x})\}
$$

Then $\Lambda_{M}(Q)<\infty$. If $\boldsymbol{x} \in B$, if $1-\delta(x) \leq t<1$ and if $\boldsymbol{r}=\boldsymbol{y}(t, \boldsymbol{x})$, then $\boldsymbol{r} \in M-Q$ and

$$
\|\mathbf{b}-\mathbf{r}\| \leq \delta(\boldsymbol{x})\|\mathbf{h}-\boldsymbol{x}\| \text {. }
$$

PROOF. According to $(6.26)$ we have

$$
\Lambda_{M}(Q) \leq \gamma_{1} \int_{x \in B} \int_{0}^{1-\delta(x)} \frac{d t}{1-t} d \mu_{B}(x)=\gamma_{1} \int_{B} \log (1 / \delta) d \mu_{B}<\infty .
$$

Also $r=x+t(b-x) \in M-Q$ if and only if $0 \leq 1-\delta(x) \leq t<1$. If so, then $\mathfrak{h}-\mathfrak{r}=(1-t)(\boldsymbol{b}-\boldsymbol{x})$ where $(1-t) \leq \delta(\boldsymbol{x})$. Hence

$$
\|\mathbf{h}-\mathbf{r}\|=(1-\mathbf{t})\|\mathbf{h}-\boldsymbol{x}\| \leq \delta(\boldsymbol{x})\|\mathbf{b}-\boldsymbol{x}\|
$$

Let $g: M \rightarrow \mathbb{R}$ and $h: M \rightarrow \mathbb{R}$ be functions. We say that $g(r) \leq h(r)$ for most $r$ in $M$ and write $g(r) \leq h(r)$ on $M$ if there exists a measurable subset $E$ of $M$ with $\hat{M}_{M}(E)<\infty$ such that $g(\mathfrak{r}) \leq h(\mathfrak{r})$ for all $\mathbf{r} \in M-E$.

LEMMA 6.12. Let $g, h, k$ and $\ell$ be real valued functions on $M$. Then

(a) If $g \leq h$ and $h \leq k$ on $M$, then $g \leq k$ on $M$.

(b) If $\mathrm{g} \leq \mathrm{h}$ and $0 \leq \mathrm{k}$ on $\mathrm{M}$, then $\mathrm{gk} \leq \mathrm{hk}$ on $\mathrm{M}$.

(c) If $\mathrm{g} \leq \mathrm{h}$ and $\mathrm{k} \leq \ell$ on $M$, then $\mathrm{g}+\mathrm{k} \leq \mathrm{h}+\ell$.

(d) If $t_{0} \in \mathbb{R}(0,1)$ and $g(r) \leq h(r)$ for all $r \in M\left[t_{0}, 1\right)$, then $g(r) \leq h(r)$.

(e) If $g(r) \rightarrow \infty$ for $r \rightarrow b$ with $r \in M$ and if $a \in \mathbb{R}$ and $0<\varepsilon \in \mathbb{R}$ then a $\leq \varepsilon(\mathbf{r})$.

(f) If $g(r) \leq h(r)$, then $\lim \inf g(r) \leq 1$ im $\sup h(r)$.

$$
M \ni \mathbf{r} \rightarrow \mathfrak{h} \quad M \ni r \rightarrow b
$$

PROOF. (a) to (e) are trivial. In the case (f), there exists a measurable set $E$ in $M$ with $\Lambda_{M}(E)<\infty$ such that $g(r) \leq h(r)$ for all $r \in M-E$. Take a sequence $\left\{t_{j}\right\}_{j \in \mathbb{N}}$ with $0<t_{j}<1$ such that $t_{j} \rightarrow 1$ for $j \rightarrow \infty$. By $(6.28), r_{j} \in M\left[t t_{j}, 1\right)-E$ exists. Then $\boldsymbol{r}_{j} \rightarrow \mathbf{b}$ for $\mathbf{j} \rightarrow \infty$ and $g\left(\mathbf{r}_{\mathbf{j}}\right) \leq \mathrm{h}\left(\mathbf{r}_{\mathbf{j}}\right)$. Therefore 


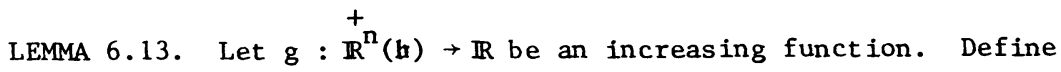
$S=\sup \left\{g(r) \mid r \in \stackrel{+}{n}^{n}(\boldsymbol{h})\right\} \leq \infty$. Then

$$
g(\mathbf{r}) \rightarrow S \text { for } \mathbf{r} \rightarrow \mathbf{h} \text { with } \mathbf{r} \in M
$$

PROOF. The function $g(r b)$ of $r \in \mathbb{R}(0,1)$ increases. Therefore $g(r b) \rightarrow s$ for $\mathbf{r} \rightarrow \infty$. Obviously $\mathbf{s} \leq \mathrm{S}$. Take $\mathrm{T}<\mathrm{S}$. A point $\mathbf{r}_{0}$ with $0 \leq \mathbf{r}_{0}<\mathbf{b}$ exists such that $\mathrm{T}<\mathrm{g}\left(\mathbf{r}_{0}\right)$. A number $\mathrm{r}_{0} \in \mathbb{R}(0,1)$ exists such that $\mathbf{r}_{0}<\mathbf{r}_{0} \mathbf{b}$. Then $T<g\left(r_{0}\right) \leq g\left(r_{0} \mathfrak{r}\right) \leq s$. Hence $s \leq s$. We find that $s=s$ and $g(r b) \rightarrow s$ for $r \rightarrow 1$ if $0<\mathrm{r}<1$

Again take $T<S$. A number $r_{0}=r_{0}(T) \in \mathbb{R}(0,1)$ exists such that $g\left(r_{0} h\right)>T$. Take $r \in M\left[r_{0}, 1\right)$. Then $r=x+t(b-x)$ with $r_{0} \leq t<1$ and $\boldsymbol{x} \in$ B. Therefore $\mathbf{r}=(1-t) \boldsymbol{x}+t \mathfrak{h} \geq t \mathbf{h} \geq r_{0} \mathbf{h}$ which implies $T<g\left(r_{0} \mathbf{h}\right) \leq g(\mathbf{r}) \leq S$ for all $\mathbf{r} \in M\left[r_{0}, 1\right)$. Hence $g(r) \rightarrow S$ for $r \rightarrow b$ if $r \in M ; q \cdot e . d$.

In order to eliminate $\theta$ we need the following well-known Lemma.

LEMMA 6.14. (Hayman [12] Lemma 2.4 p. 38) Take $t_{0} \in \mathbb{R}[0,1$ ). Let $\mathrm{T}: \mathbb{R}\left[\mathrm{t}_{0}, 1\right) \rightarrow \mathbb{R}$ be a continuous, increasing function. Assume that there is a positive number $c>0$ such that $T(t) \geq c$ for all $t \in \mathbb{R}\left[t_{0}, 1\right)$. Define

$$
E=\left\{t \in \mathbb{R}\left[t_{0}, 1\right) \mid T\left(t+\frac{(1-t) c}{e T(t)}\right)>2 T(t)\right\}
$$

Then $E$ is open in $\mathbb{R}\left[t_{0}, 1\right)$ with $\int_{E}(1 / 1-t) d t \leq 2$.

PROPOSITION 6.15. Define $Q$ by $(6.29)$. Let $T: M \rightarrow \mathbb{R}$ be a continuous, increasing function. Assume that there are constants $c>0$ and $s \in \mathbb{R}(0,1)$ such that $T(r) \geq c$ for $r \in M[s, 1)$. Define $T_{0}=\operatorname{Max}(c, T)$ as a function on $M$. A continuous function $\mathfrak{p}: M \rightarrow \mathbb{R}^{\mathrm{n}}$ is defined by

$$
\mathfrak{p}(\mathfrak{r})=\mathfrak{y}\left(t+\frac{(1-t) c}{e T_{0}(\mathfrak{r})}, \boldsymbol{x}\right)
$$

for all $\boldsymbol{r} \in M$ where $(t, \boldsymbol{x}) \in \mathbb{R}(0,1) \times B$ is uniquely determined by $\mathbf{r}=\boldsymbol{y}(t, \boldsymbol{x})$. Then $\mathfrak{r}<\mathfrak{p}(\mathfrak{r})<\mathfrak{b}$ for $\mathfrak{r} \in M$ and $\mathfrak{p}(\mathfrak{r}) \in \stackrel{+}{\mathbb{R}}^{n}(\mathfrak{h})$. Let $\Theta(\mathfrak{r})$ be the largest number such that 
$\theta(\mathfrak{r}) \mathfrak{r} \leq \mathfrak{p}(\mathfrak{r})$ for $\mathfrak{r} \in M$. Then $\theta(\mathfrak{r})>1$. Define $c_{0}=\log \left(8\|\mathfrak{b}\|^{2} / c s\right)$. Then

$$
\log \frac{2 \Theta(\mathfrak{r})}{\Theta(\mathfrak{r})-1} \leq \log T(\mathfrak{r})+2 \log \frac{1}{\|\mathfrak{b}-\mathfrak{r}\|}+c_{0}
$$

for all $\mathbf{r} \in \mathrm{M}[s, 1)-\mathrm{Q}$. In particular

$$
\log \frac{2 \Theta(\mathfrak{r})}{\Theta(\mathfrak{r})-1} \leq \log T(\mathfrak{r})+2 \log \frac{1}{\|\mathfrak{h}-\mathfrak{r}\|}+\mathrm{c}_{0}
$$

on M. Define $\gamma_{0}=\operatorname{Min}\{\|\mathbf{h}-\boldsymbol{x}\| \mid \boldsymbol{x} \in \overline{\mathrm{B}}\}>0$ and

$$
\gamma_{3}=\mu_{B}(B) \gamma_{0}^{-p}\left(2+\log \frac{1}{1-s}\right)
$$

Then there exists an open subset $E$ of $M$ with $\Lambda_{M}(E) \leq \gamma_{3}<\infty$ such that

$$
T(\mathfrak{p}(\mathfrak{r})) \leq 2 \mathrm{~T}(\mathfrak{r}) \quad \text { for all } \mathfrak{r} \in \mathrm{M}-\mathrm{E} .
$$

In particular $T(\mathfrak{p}(\mathfrak{r})) \leq 2 \mathrm{~T}(\mathfrak{r})$ on $M$.

PROOF. Trivially $\mathrm{T}_{0}$ is continuous and $\mathrm{T}_{0} \geq \mathrm{c}$ on $\mathrm{M}$. Take $\mathfrak{r} \in \mathrm{M}$. Then $\mathfrak{r}=\mathfrak{y}(t, x)$ with $0<t<1$ and $\boldsymbol{x} \in$ B. Hence

$$
\begin{aligned}
& 0<t<t+\frac{(1-t) c}{e T(r)}<1 \\
& 0<\mathfrak{r}=\mathfrak{y}(t, x)<\mathfrak{p}(\mathfrak{r})<\mathfrak{y}(t, \boldsymbol{x})<\mathfrak{b} .
\end{aligned}
$$

Consequently, $\Theta(\mathfrak{r})>1$ and $\mathfrak{p}(\mathfrak{r}) \in \stackrel{+}{\mathrm{n}}^{\mathrm{n}}(\mathfrak{b})$. Take $\mathfrak{r} \in \mathrm{M}[\mathrm{s}, 1)$. Then $\mathfrak{r}=\boldsymbol{x}+\mathfrak{t}(\mathfrak{b}-\boldsymbol{x})$ with $x \in B$ and $s \leq t<1$. Also

$$
\mathfrak{p}(\mathfrak{r})=\boldsymbol{x}+\left(t+\frac{(1-t) c}{\mathrm{eT}(\mathfrak{r})}\right)(\mathfrak{b}-\boldsymbol{x})=\mathfrak{r}+\frac{(1-t) c}{\mathrm{eT}(\mathfrak{r})}(\mathfrak{b}-\boldsymbol{x})
$$

For each $j \in \mathbb{N}[1, n]$ we have

$$
\theta(\mathfrak{r}) r_{j} \leq r_{j}+\frac{(1-t) c}{e T(r)}\left(b_{j}-x_{j}\right)
$$

with equality for at least one $j$. Since $r_{j}>0$ we obtain 


$$
\Theta(\mathfrak{r})=1+\frac{(1-t) c}{\operatorname{eT}(\mathfrak{r})} \operatorname{Min}_{1 \leq j \leq n} \frac{b_{j}-x_{j}}{r_{j}}
$$

We have $\mathbf{b}-\mathbf{r}=(1-t)(\mathfrak{h}-\boldsymbol{x})$. Hence $\|\mathbf{b}-\mathfrak{r}\|=(1-t)\|\mathbf{b}-\boldsymbol{x}\|$ and

$$
\theta(\mathfrak{r})=1+\frac{\|\mathfrak{h}-\mathfrak{r}\|}{\|\mathfrak{b}-\boldsymbol{x}\|} \frac{c}{\mathrm{eT}^{T(\mathfrak{r})}} \operatorname{Min}_{1 \leq \mathbf{j} \leq n} \frac{\mathrm{b}_{\mathbf{j}}-\mathrm{x}_{\mathbf{j}}}{\mathrm{r}_{\mathbf{j}}}
$$

where $r_{j}=x_{j}+t\left(b_{j}-x_{j}\right) \geq s\left(b_{j}-x_{j}\right)$. Hence

$$
\theta(\mathfrak{r}) \leq 1+\frac{1}{\mathrm{se}}=\frac{\mathrm{se}+1}{\mathrm{se}} \leq \frac{4}{\mathrm{se}} .
$$

Also $r_{j}=(1-t) x_{j}+t b_{j}<(1-t) b_{j}+t b_{j}=b_{j}$. Hence

$$
\begin{gathered}
\operatorname{Min}_{1 \leq j \leq n} \frac{b_{j}-x_{j}}{r_{j}} \geq \underset{1 \leq j \leq n}{\operatorname{Min}} \frac{b_{j}-x_{j}}{b_{j}}=\delta(x) \\
\theta(r) \geq 1+\frac{\|b-r\|}{\|b-x\|} \frac{c}{e T(r)} \delta(x) .
\end{gathered}
$$

Now assume that $r \in M[s, 1)-Q$. Then $1>t \geq 1-\delta(x)$ by (6.29). Consequently

$$
\delta(\boldsymbol{x}) \geq 1-\mathrm{t}=\frac{\|\mathfrak{b}-\mathfrak{r}\|}{\|\mathfrak{b}-\boldsymbol{x}\|}
$$

Since $\|\mathbf{b}-\boldsymbol{x}\| \leq\|\mathfrak{b}\|$, we obtain

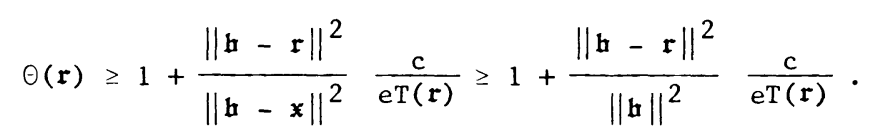

Now (6.39) and (6.41) imply

or

$$
1 \leq \frac{2 \Theta(\mathfrak{r})}{\Theta(\mathbf{r})-1} \leq \frac{8\|\mathfrak{b}\|^{2}}{\mathrm{sc}} \frac{\mathrm{T}(\mathbf{r})}{\|\mathfrak{b}-\mathbf{r}\|^{2}}
$$

$$
0 \leq \log \frac{2 \Theta(\mathfrak{r})}{\Theta(\mathfrak{r})-1} \leq \log \mathrm{T}(\mathfrak{r})+2 \log \frac{1}{\|\mathbf{b}-\mathbf{r}\|}+\log \frac{8\|\mathbf{b}\|^{2}}{\mathrm{sc}}
$$

for all $\mathfrak{r} \in \mathrm{M}[\mathrm{s}, 1)-\mathrm{Q}$, which proves (6.33). Now (6.28) and Lemma 6.11 prove (6.34). 
The open set $E$ is defined by $E=\{r \in M \mid T(\mathfrak{p}(\mathfrak{r}))>2 T(\boldsymbol{r})\} \cup M[0, s)$. Then $T(\mathfrak{p}(\boldsymbol{r})) \leq 2 T(\boldsymbol{r})$ for al1 $\boldsymbol{r} \in M-E$. For $\boldsymbol{x} \in \mathrm{B}$ and $t \in \mathbb{R}(0,1)$ define $T_{\boldsymbol{x}}(t)=T(\boldsymbol{y}(t, \boldsymbol{x}))$. Define

$$
E(x)=\left\{t \in \mathbb{R}[s, 1) \mid T_{x}\left(t+\frac{(1-t) c}{e^{T}(t)}\right)>2 T_{x}(t)\right\} \cup \mathbb{R}[0, s) .
$$

According to Lemma 6.14 we have

$$
\int_{E(x)} \frac{d t}{1-t} \leq 2+\log \frac{1}{1-s} .
$$

Therefore Lemma 6.10 implies

$$
\Lambda_{M}(E) \leq \mu_{B}(B) \gamma_{0}^{-p}\left(2+\log \frac{1}{1-s}\right)=\gamma_{3}<\infty
$$

LEMMA 6.16. Let $\mathrm{f}$ be a non-constant meromorphic function on $\mathbb{D}(\mathfrak{b})$. Then $\mathrm{T}_{\mathrm{f}}(\mathbf{r h}, \mathbf{s h})>0$ if $0<\mathrm{s}<\mathrm{r}<\mathrm{l}$

PROOF. If $f$ has a point of indeterminacy at 0 , then $\imath_{f}(0)>0$ and $(5.17)$ implies that $T_{f}(r \mathfrak{r}, s \mathfrak{s}) \geq l_{f}(0) \log r / s>0$. Hence we can assume that $f$ is holomorphic at 0 . If $\mathrm{T}_{\mathrm{f}}(\mathrm{rb}, \mathrm{sh})=0$ for some pair $0<\mathrm{s}<\mathrm{r}<1$, identity (5.14) implies $A_{f}(t h)=0$ for $s \leq t \leq r$. Then (5.5) and (5.13) show that $A_{f[z]}(t)=0$ for almost all $z \in \mathbb{D}\langle\boldsymbol{h}\rangle$ and $s \leq t \leq r$. Hence $f[z]$ is constant on $\mathbb{D}(t)$ and therefore on $\mathbb{D}$ (1) for almost all $z \in \mathbb{D}\langle\mathfrak{h}\rangle$. By continuity $f[z]$ is constant for all $z \in \mathbb{D}\langle\mathbf{b}\rangle$. Let

$$
f(z)=\sum_{\lambda=0}^{\infty} P_{\lambda}(z)
$$

be the development into homogeneous polynomials at 0 . Then

$$
f(z z)=\sum_{\lambda=0}^{\infty} P_{\lambda}(z) z^{\lambda}
$$

for all $z \in \mathbb{C}^{\mathrm{n}}$ and $z \in \mathbb{C}$ sufficiently small. Because $f(z z)$ is constant for $z \in \mathbb{D}\langle\mathfrak{b}\rangle$ and $z \in \mathbb{D}(1)$ we have $\mathrm{P}_{\lambda} \mid \mathbb{D}\langle\mathfrak{h}\rangle \equiv 0$ if $\lambda>0$. Hence $\mathrm{f} \equiv \mathrm{P}_{0}$ is constant.

Contradiction! q.e.d.

LEMMA 6.17. Let $\mathrm{f}$ be a non-constant meromorphic function on $\mathbb{D}(\mathrm{b})$. Take $\mathfrak{q} \in \mathbb{R}^{+r_{\mathbf{l}}}(\mathfrak{h})$ and $q>0$ with $q \mathfrak{h}<\mathfrak{q}$. Then there exist numbers $\mathbf{c}>0$ and $\mathbf{s} \in \mathbb{R}(0,1)$ such that $\mathbf{r}>\mathbf{q}$ and $\mathrm{T}(\mathbf{r}, \mathfrak{q}) \geq c$ for all $\mathfrak{r} \in \mathrm{M}[\mathbf{s}, 1)$. 
PROOF. A number $\mathrm{q}_{0} \in \mathbb{R}(\mathrm{q}, 1)$ exists such that $\mathbf{q}<\mathrm{q}_{0} \mathbf{b}<\mathbf{b}$. Take $\mathbf{s} \in \mathbb{R}\left(\mathrm{q}_{0}, 1\right)$. By Lemma $6.16, c=T_{f}\left(s h, q_{0} \mathfrak{h}\right)>0$. Take $r \in M[s, 1)$. Then $\mathbf{r}=\boldsymbol{x}+\mathrm{t}(\boldsymbol{h}-\boldsymbol{x})=(1-t) \boldsymbol{x}+\mathrm{th}$ with $\boldsymbol{x} \in \mathrm{B}$ and $\mathrm{s} \leq \mathrm{t}<1$. Hence $\mathbf{r}>\mathrm{sh}>\mathrm{q}_{0} \mathbf{b}>\mathbf{q}$ and

$$
T(\mathbf{r}, \mathfrak{q}) \geq T(s \mathfrak{h}, \mathfrak{h}) \geq T\left(s \mathfrak{h}, q_{0} \mathfrak{h}\right)=c>0
$$

Now, the Lemma of the logarithmic derivative follows easily, which constitutes the main result of this paper.

THEOREM 6.18. Take $\mathfrak{b} \in \stackrel{+}{\mathbb{n}}^{n}$ and $\mathfrak{q} \in \stackrel{+}{n}^{n}(\mathfrak{l})$. Let $\mathrm{f}$ be a non-constant meromorphic function on $\mathbb{D}(\mathbf{b})$. Let $M$ be an approach cone. Take $\tau \in \mathbf{N}[1, n]$. Then

$$
\int_{\mathbb{D}\langle\mathbf{r}\rangle} \log ^{+}\left|\frac{\mathrm{df}}{\mathrm{d} \mathbf{z}_{\tau}} / \mathrm{f}\right| \Omega^{\mathrm{n}} \leq 17 \log ^{+} \mathrm{T}_{\mathrm{f}}(\mathbf{r}, \mathbf{q})+19 \log ^{+} \frac{1}{\|\mathbf{r}-\mathbf{r}\|}
$$

for $r \in M$.

PROOF. Take $\mathrm{q}>0$ with $\mathrm{qh}<\mathrm{q}$. Then $\mathrm{c}>0$ and $\mathrm{s} \in \mathbb{R}(0,1)$ exist such that $\mathbf{r}>\mathfrak{q}$ and $T(\mathbf{r}, \mathfrak{q}) \geq c$ for all $\mathfrak{r} \in M[s, 1)$. For $T(\mathbf{r}, \mathfrak{q})$ define $\mathfrak{p}: M \rightarrow \mathbb{R}^{n}$ by $(6.32)$ and $\Theta(r)$ as the largest number such that

$$
\mathfrak{r}<\theta(\mathfrak{r}) \mathbf{r} \leq \mathfrak{p}(\mathfrak{r})<\mathfrak{h} \quad \text { for } \mathbf{r} \in M
$$

Then (6.34) and (6.36) hold for $T(\mathfrak{r})=T(\mathfrak{r}, \mathfrak{q})$. Also we have (6.12). Let $c_{j}$ be positive constants. Take $\mathfrak{q} \leq \mathfrak{r}<\mathfrak{h}$. Then

$$
\begin{aligned}
& \int_{\mathbb{D}\langle\mathfrak{r}\rangle} \log ^{+}\left|\mathrm{f}_{\tau}^{\prime} / \mathrm{f}\right| \Omega_{\mathrm{n}} \leq 8 \log ^{+} \mathrm{T}_{\mathrm{f}}(\Theta(\boldsymbol{r}) \mathfrak{r}, \mathfrak{q})+9 \log \frac{2 \Theta(\mathbf{r})}{\Theta(\mathfrak{r})-1}+c_{1} \\
& \leq 8 \log ^{+} \mathrm{T}_{\mathrm{f}}(\mathfrak{p}(\mathfrak{r}), \mathfrak{q})+9 \log \frac{2 \Theta(\mathfrak{r})}{\Theta(\mathfrak{r})-1}+c_{1} \\
& \leq 8 \log ^{+}\left(2 \mathrm{~T}_{\mathrm{f}}(\mathbf{r}, \mathfrak{q})\right)+9 \log \mathrm{T}_{\mathrm{f}}(\mathbf{r}, \mathfrak{q})+18 \log \frac{1}{\|\mathbf{b}-\mathbf{r}\|}+c_{2} \\
& \leq 17 \log ^{+} \mathrm{T}_{\mathrm{f}}(\mathfrak{r}, \mathfrak{q})+19 \log ^{+} \frac{1}{\|\mathfrak{b}-\mathfrak{r}\|}
\end{aligned}
$$

where the constant is swallowed by $\log (1 /\|\mathbf{b}-\mathbf{r}\|)$ (see Lemma 6.12e) q.e.d.

Now, it is possible to derive the defect relation along the lines of Vitter [5] and Sto11 [6]. 
Naturally, Theorem 6.18 extends immediately to any differentiable operator

$$
D=\sum_{j=1}^{n} A_{j} \frac{\partial}{\partial z_{j}}
$$

where the $A_{j}$ are bounded continuous functions. If we want $\mathrm{Df} / \mathrm{f}$ to be meromorphic, we have to take $A_{j}$ as bounded holomorphic functions on $\mathbb{D}(\boldsymbol{b})$. We even could take $A_{j}$ unbounded, if we add a correction term on the right-hand side in (6.42). If the $\mathrm{A}_{j}$ are bounded holomorphic functions on $\mathbb{D}(\boldsymbol{h})$, then $\mathrm{Df} / \mathrm{f}$ is meromorph $1 \mathrm{c}$. We have

$$
\begin{gathered}
\mathrm{m}_{\mathrm{Df} / \mathrm{f}}(\mathfrak{r}, \infty)=\int_{\mathbb{D}\langle\mathbf{r}\rangle} \log \sqrt{1+|\mathrm{Df} / \mathrm{f}|^{2}} \Omega_{\mathrm{n}} \leq \int_{\mathbb{D}\langle\mathbf{r}\rangle} \log ^{+}|\mathrm{Df} / \mathrm{f}| \Omega_{\mathrm{n}}+\log 2 \\
\mathrm{~m}_{\mathrm{Df} / \mathrm{f}}(\mathbf{r}, \infty) \leq 17 \mathrm{n} \log ^{+} \mathrm{T}_{\mathrm{f}}(\mathbf{r}, \mathfrak{q})+19 \mathrm{n} \log ^{+} \frac{1}{\|\mathfrak{h}-\mathfrak{r}\|}
\end{gathered}
$$

if the constant $c_{2}$ in the proof includes $n \log 2$ and $n \log C$ where $C$ is an upper bound of the $A_{j}$.

ACKNOWLEDGEMENT. This paper was supported in part by the National Science Foundation Grant No. 80-03257.

\section{REFERENCES}

1. RONKIN, L.I. Introduction to the Theory of tntire tunctions of Several Variables, translation of Math. Monographs Amer. Math. Soc. 44, 273 pp., 1974.

2. STOLL, W. Holomorphic Functions of Finite Order in Several Complex Variables, Reg. Conf. Series 21,83 pp., 1974.

3. HENSON, C.W. and RUBEL, L.A. Solution of Tarski's 'High School Algebra Problem' for a Restricted Class of Exponential Polynomials I (One Variable) Notices Amer. Math. Soc.

4. HENSON, C.W. and RUBEL, L.A. Solution of Tarski's 'High School Algebra Problem' for a Restricted Class of Exponential Polynomials II (Several Variables) Notices Amer. Math. Soc.

5. VITTER, A. The Lemma of the Logarithmic Derivative in Several Complex Variables Duke Math. J. 44, 89-104, 1977.

6. STOLL, W. Introduction to Value Distribution Theory of Meromorphic Maps, Complex Analysis, Proceedings, Trieste 1980. Lecture Notes in Mathematics 950, Springer-Verlag 1982, p. 210-359.

7. GAUTHIER, P.M. and HENGARTNER, W. The Value Distribution of Most Functions of One or Several Complex Variables Ann. Math. 96, (1972) 31-52.

8. GRIFFITHS, Ph. and KING, J. Nevanlinna Theory and Holomorphic Mappings Between Algebraic Varieties Acta Math. 130, (1973) 145-220.

9. BIANCOFIORE, A. and STOLL, W. Another Proof of the Lemma of the Logarithmic Derivative in Several Complex Variables Recent Developments in Several Complex Complex Variables, Princeton University Press, 29-45, 1981. 
10. VLADIMIROV, V.S. Methods of the Theory of Functions of Many Complex Variables, translated by L. Ehrenpreis, M.I.T. Press, 353 pp. , 1966.

11. ANDREOTTI, A. and STOLL, W. Analytic and Algebraic Dependence of Meromorphic. Functions, Lecture Notes in Mathematics 234, Springer-Ver1ag, 390 pp., 1971.

12. HAYMAN, W.K. Meromorphic Functions, Oxford University Press, 191 pp. 1964.

13. STOLL, W. Die beiden Hauptsatze der Wertverteilungstheorie bei Funktionen mehrerer komplexer Veranderlichen I Acta Math. 90 (1953), 1-115. "II" Acta Math. 92 (1954), 55-169.

14. STOLL, W. Deficit and Bezout Estimates, Value-Distribution Theory Part B, (edited by Robert I. Kujala and Albert L. Vitter III), Pure and Appl. Math. 25, Marcel Dekker, New York, 271 pp., 1973.

15. STOLL, W. Value Distribution on Parabolic Spaces, Lecture Notes in Mathematics 600, Springer-Verlag, 216 pp., 1977.

16. STOLL, W. Ahlfors-Weyl Theory of Meromorphic Maps on Parabolic Manifolds, Value Distribution Theory, Proceedings, Joensuu 1981. Lecture Notes in Mathematics 981 Springer-Verlag 1983, p. 101-219. 


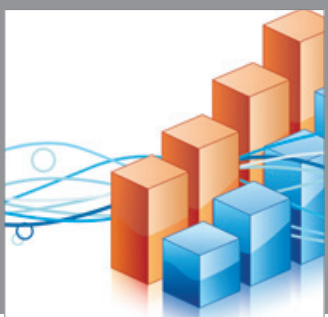

Advances in

Operations Research

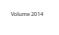

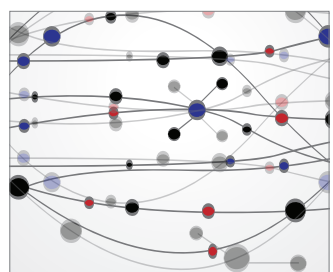

\section{The Scientific} World Journal
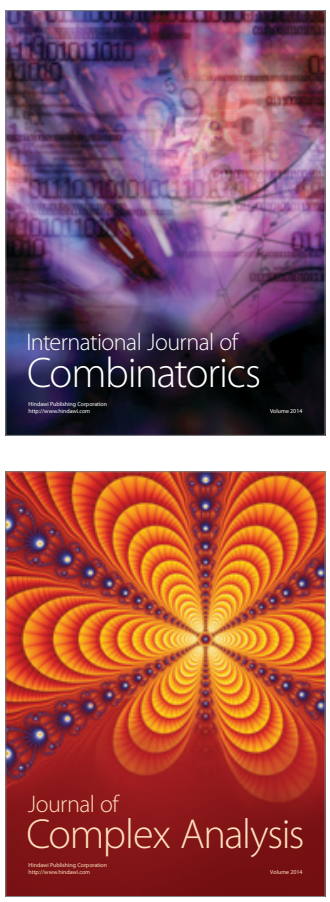

International Journal of

Mathematics and

Mathematical

Sciences
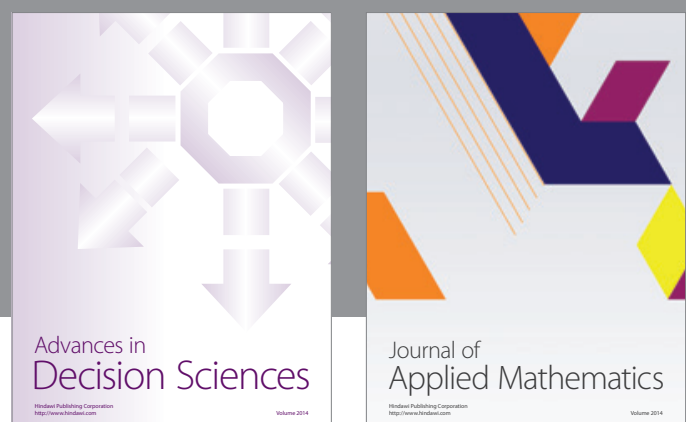

Journal of

Applied Mathematics
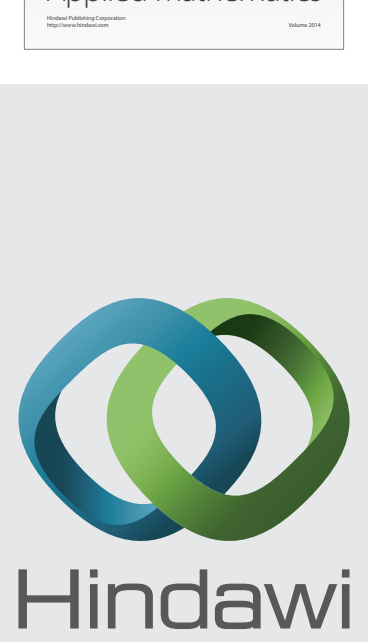

Submit your manuscripts at http://www.hindawi.com
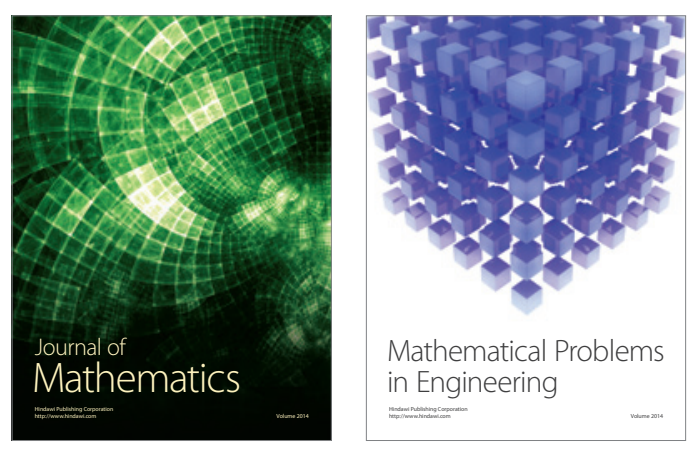

Mathematical Problems in Engineering
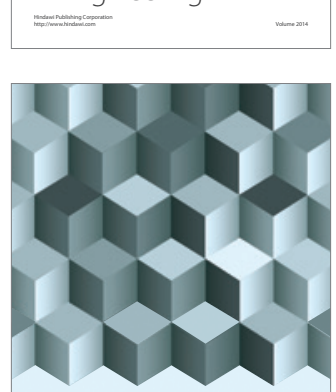

Journal of

Function Spaces
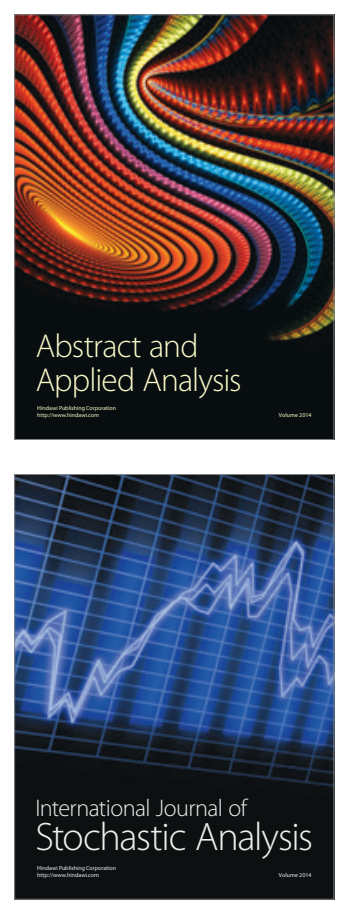

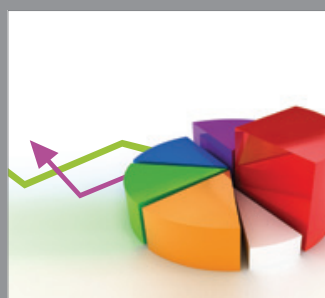

ournal of

Probability and Statistics

Promensencen
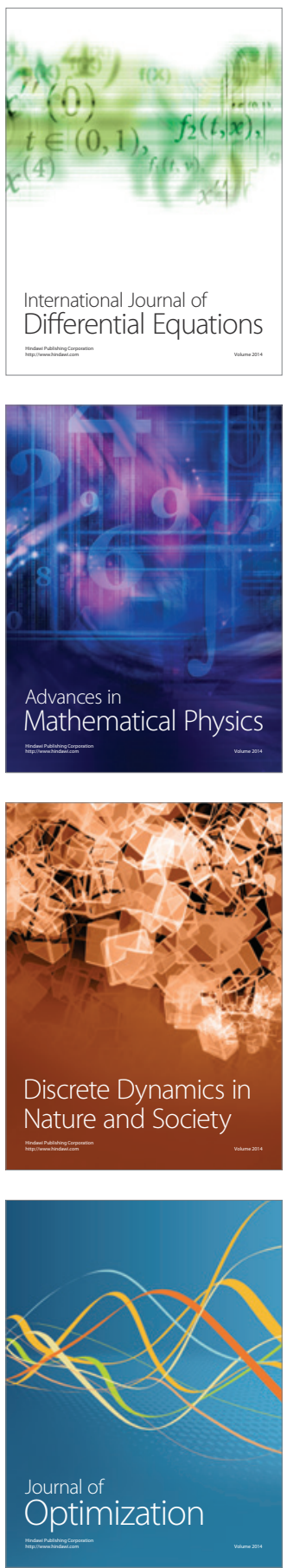